\title{
Nuclear Reactors Built, Being Built, or Planned: 1995
}

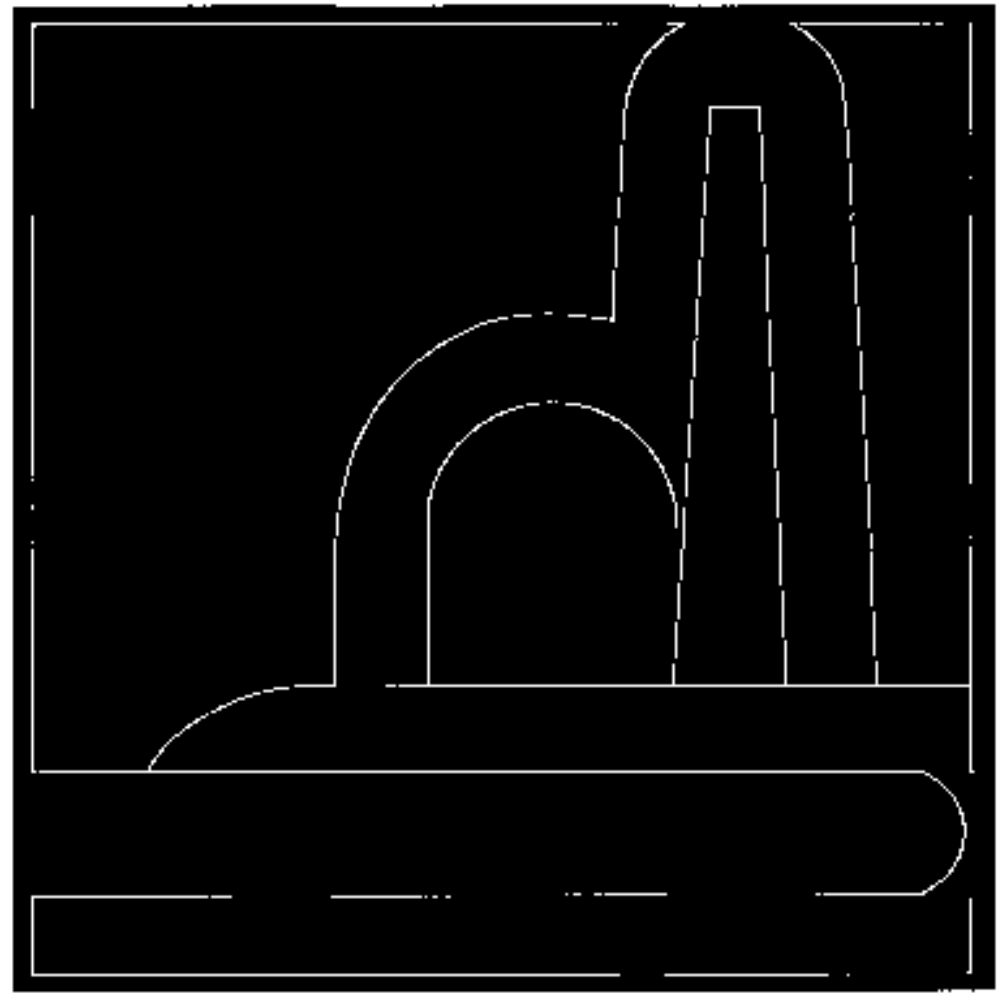

Prepared for:

U.S. DEPARTMENT OF ENERGY

Director, Office of Nuclear Energy,

Science and Technology

Prepared by:

Office of Scientific and

Technical Information 
Avajlable to DOE and DOE contractors from the Office of Scientifice and Technical Information, P.O. Box 62, Oak Ridge, Tennessee 37831; prices available from (423) 576-84i)1.

Available to the public from the U.S. Department of Commerce, Technology Administration, National Technical Information Service, Springfield, Virginia 22161; prices available from (703) 487,4650 . 


\section{Nuclear Reactors Built, Being Built, or Planned

This teport was prepared as an accontent or work spoosoced by an asency of the Unted Siates

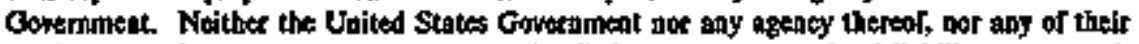
amployees, makes any warranty, express os tomplied, or asurues any legal liability or rwpongibility for the eccurecy, completeness, or usefulness of any irformation, apparstus, produch, or process dischosed, or represeats that fis use woukd nor InIringe privately orfaed rights Refer-

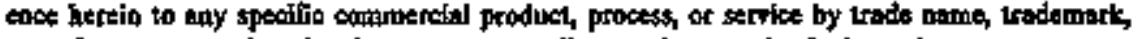
manufacturet, or otherwise does nol oecestarify constilute ar imply its endorsemenl, recom. mendation, or faroring by tho United States Ooretament or any ageacy thereal. The vats and opinions of aothos expressed bervin do sot necessarily slate of reflect those of the Uniled Sertes Goverament or any ayancy thereof.

Prepared for:

\section{U.S. DEPARTMENT OF ENERGY}

Director, Office of Nuclear Energy, Science and Technology

Washington, 0.C. 20585

Prepared by:

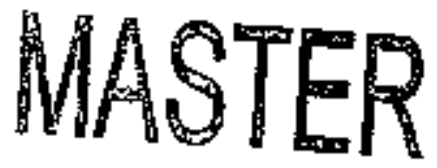

Office of Scientific and Technical Intormation

DISTRIBUTION OF THFS DOCUMENT IS UNLIMTED 


\section{DISCLADMER}

Portions of this document may be illegible in electronic image products. Images are produced from the best available original document. 


\section{NOTICE \\ Change of Format}

Effective August 1, 1996, Nuclear Reactors Built, Being Built, or Planned: 1995 (DOENOSTI-8200-R59) will be made available on the Office of Scientific and Technical Information Home Page on the World Wide Web (WWW) at the Uniform Resource Locator (URL)

http:/wwwddoe.gov/osti/ostipg.html

Select the link "Products and Services" and then select the link "Publications." At "Publications" select the title Nuclear Reactors Buit, Being Buil, or Planned: 1995 (DOE/OSTI-8200).

The direct URL for the DOE/OSTI--8200 Web page is

htt://www.doe.gov/osti/products/nrb.html

Between August 1, 1996, and June 1997 this data will be available both in the traditional printed form as well as elextronically. However, begining with the 1997 issue, distribution of the printed copies will be discontinued.

The WWW version of DOE/OSTI-8200-R59 will be available in Adobe Acrobat Portable Document Fonmat (PDF) and, using a graphical WWW browser such as Netscape or Mosaic, can be viewed and printed throngh the Adobe Acrobat Reader. Instructions for dowbloading the Acrobat Reader and integrating it with your browser are provided via hyperlink at the DOE/OSTI- 8200 Web page, or by pointing your browser to

http:/www.adobe.com/

If you have difficulty downloading the Acrobat ${ }^{\oplus}$ Reader you may send E-mail to webmaster@zeus.osti.gov

In the event that access to this data through the WWW is not an option for you, you may contact the Department of Energy Technical Infotmation Service at 423-576-8401 or send E-mail to

reports@adonis.osti.gov 


.




\section{Preface}

Neclear Reactors Buit, Reing Buth, or Plaraned contains miclassiried information about facilittes built, being built, or plannet in the Unitod States for fomestic use or export as of December 31, 1995. The Office of Scientific and Technical Information, U.S. Department of Energy, pathers this infortur tion arnually from Washington beadquarters and licld offices of DOE; from the U,S.Nuckar Regulatory Comsisston (NRC);

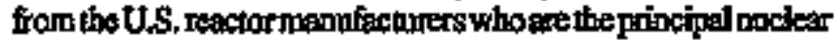
conltractors for foreizn reactor loxations; fion U.S. and fureign embassies; and from fonejgn governmental molez depactinents.

The book consists of three divisions, $x$ follows:

- a commercial reactor locator unap and talles of the characteristic and statistical data that follow; a teble of abbrevintions;

- tables of data for reactors operating, being bult, of plannect; and

- lables of data for reactors that have beer stut down permanently or dismantled.

The reactors are subdivided into the following parts: Civilian, Production, Milisery, Export, and Cirical Assembly. Export reactor efers to a reactor for whto the principal urelearcontracecor is a U.S. company-wodking eitter independently or in cooperation with a foreign congany (Part IV). Critical assembly refers $\omega$ an assembiy of fuel and moderator that requines an oxtemal source of aevitrons to infitate and maintain fission, A critcal assernbly is used for experimentalneaswements(Part V).

Various classes of reactors within these perts are defined as follows:

Central-Station Electric Power Plant A nuckear power facility designed and consuscted for operation on a utility system (Part I, Section 1A).

Dual-Purpose Plapt: A nuckear power facility desigped, constucted, and operated for more than coe primary purpose, for exaraple, the production of nuclegr onaterials and the generation of electricity or the use of reactor thermal energy for electrical genteration and process-heak applications including desalting (Part I, Section 1B), -

Experimental Power Refctor: A facility deslgoed, eng: neered, constructed, ind operated to test the technical feasifility of a concept or to provide the techuical basis for a sintilar type nuclear power plant in a larger size. Design flexibility penrits changes to prowe varions aspects of reactor technology includting fued, components, and configurations. Power-conversion expipment may or may not be includect as part of the fincility (Part I, Section 2).

General Irradiation Test Reactor: A reactor having (1) a thermal power lovel exceeding IO MW; (2) test loops or experimenlal factibites within, or in proximlty to, thecores and(3) the tse of reclear raliation for testing the life or performance of reactor componeuts as its major function (Pat I, Section 3A; Part IV, Section 2A).

Figh-Power Research and Test Reactor: A reactor biving a relarively high themral power level (5MW or greater) bout not ctassed as a genteral irradiation testreactor' (Peart I, Section 3B).

Safety-Research and Test Reactor: A rector associated wilh a nuclear safery research or enginetering-sealo test program corducted for the purpose of deretloping basic design information or demonstratingsefety charateristies of entestrialandaerospace muclear neactor sysens (Part L, Section 3C).

General Reseorch Reactori A reactor-exelutuing that lo. catedatatuiversity - whosenucleartidiationsareused primarily as a reseanch tool forbasic or agplied research and whose thernn' power Invel is 10 MW or less. Itmay inctude facilities for testimg Inector nuaterials (Pant I, Section 3D; Part IV, Section 2B). Also included ane Resezuch Reactors (Part III, Section 3B).

University Research and Teaching Reactor: A reactor lockated at a uriversity and ustully operated for the primary purpose of training in the operation and utilization of reactors and for insterction in reacter theory and performance (Pant! Section 3E; Part TV, Section 2C).

Reactors are further grouped acconting to statas:

Reators are listed as operable under the following circunstances:

I. Reactors regulated by the NRC

- when an cpersatiog license is issued.

- wheo a resclor is tempontarily shut down because of echuticel resons, modificetions, or refineling.

2. Federsl Gowernment reactors

- when criticality is achieved.

- whena rectcr is ternporarily shatdown for safety improvements.

3. Reactors for export

- when criticality is achieved.

Reactors are listed as being built wnder the following circumstances:

1. Reactors regulated by NRC

- when a construction permit is issued

- when linited work auncrization is issted.

2. Federal Government reactors

- when ground is troken.

- when components are oritered

- when a construction contract is awarded 
3. Reactors for export

- when an application for an expont license is received by NRC.

- when retiable information is received relating to the falprication of reactor compotents.

Reactors are listed as planned wader the following cilcurnstances:

1. Reactors regulated by NRC

- when a public annowncement that inclndes the primeipal verdorsupptiter ts made by the sponsoring organtaration.

- when an application for acconstruetion permit is received by NRC.

2. Federal Goverment reactors

- wher a pmbilc annourcement is mafte by the ageincy unvolved.

- when the project is otherwise appoppriately authorized.

3. Reactors for export

- when a public annomcernent thatincludes pritcipa contractor and resctor typo is made.

- when NRC receives infornution that a U.S. reactor manufacturer is proceeding with precontsurction desiggl and developinent on the basis of a letter of intent.

Reactors aro considered to be shuldown or dismanted unter the foillowing circumstances:

1. Reactors rofgulated by NRC

- when the license has appliad to the Commission For authority to surnender" a license veluntarity and to distuantle the facility and dispose of its componentparts. A resctor tiout down because of technical protetens, modiffications, or refieling. continues io be listed as operable.

2. Fedital Goverrment reactors

- when the facility has ceased operation and the agency has declared officially that the agency does not intend to oparate the reactor further. However, willin thiscaregory, a few itactors are identifedas being in standby mode, thecondition in whict documentany antionization exists to maintain thereactor forpossible finmereperation.
3. Reactors for export

- when the plant is officially dectared stant dowe by the owner and taken out of ogeration permanently.

Totle 2 presents a staistical stanumy of reactors, other than critteal assemotilies, ineach class and stafns. Shutdowe and dismantled reactors are included since suct facilitios have made significant contributions to reactor technology.

The reactor tebles have the following column headings:

- Location. The ciry and state or couritry where bocaled crigirally. Fort a porlable facility or one that has bees relocated, the most recent locetion is given.

- Prizcipal huclear contractor, aperator, desigat, shipbrilder. The albreviations used in this column are spelled ont in Table 3, which appeas just before the reactor tables.

- Type. Entries in this collumn are based on coolant, noderator, and nentroa energy.

- Fower. MD capacity [MW(e)] is the maximum dependabte capacity (net electrical outpot to griid) for plents having an operating hastory. Otherwiss, it is the destgrs capacily. Litcensed power and authorized power aro Fivex where afprophiare.

- Destignosion. The common name, abbreviation or acrogyn used for the facilty. For the naval reactors, it is the bull number.

- Dase columns. The initial critixality date, year of operation, and year of shutdown are given as approyntalo.

Nuclear Reacrors Buft, Being Bulh, or Planned (DOEI OSTI - 8202-R59) is sponsored by the DOE Office of Nuclear Energy, Science and Techuology, LaRue E. Moxley, Program Officer.

The participstion and assistsmee of many individuals, agencies, and companies in providing daka and updating the entries in this revision are gratefully ackorowledged. Comrments and suggestions about tris publication are welcome. To ensme that the wide range of information included in this publication will continnes to be timely aad aceurato, pitease ditrectany informen ton related to updaling the iterns to Janice Pitsenbarger, U.S. Department of Energs, Office of Stientific and Technical Information, P.O. Box 62, Oak Ridge, TN 37831; (423) $576-5636$. Questions of a ectrnical nalure should be auditressed so Lamar Cason at the same adidress. 
Table 1. Commercial Nuclear Power Reactors in the United States

Table 2. Statistical Summary of Nuciear Reactors as of 31 Dec 1995 Abbresiations of Coatractors, Designters, Shipbuilders, and Facility Openators

\section{BEACTORS AND FACILTIES}

\section{OPERABLE, BEING BUILT, OR PLANNED}

Part l. Civilian Reactors (Domestic)

1. Power Reactors
A. Central-Station Electric Power Plents
B. Dual-Purpose Plants iNo reactors currentily in this castegory\})
C. Propulsion (Maritime) No reactors cunently ts shis category)

2. Experinucatal Power-Reactor Systems
A. Electric-Power Systens (Wo reactors cerrently in this cotegory)
B. Space Nuclear Awilliary Power (SNAP) (No reactors curently in this category)
C. Spece Propulsion (No reactors currently in this category)

3. Test, Research, and Universily Reactors
A. Genefal Irradiation Test
B. High-Power Research and Test
C. Safey Reserch and Test (No reactors currendy in this cotegory)
D. General Resegrct
E. University Research aad Teacting

Part 11. Production Reaxtors

1. Materials Production

2. Process Development (No reactors currently in this category) 


\section{CONTENTS (Continued)}

Part III. Milttary Reactors

1. Detense Power-Reactor Applications

A. Renote Installations (No reactors currently in this category) I1

B. Propmision (Navai)

2. Devekpmental Powter

A. Electric-Power Experiments and Prototypes (No reactors currently It this category)

B. Propulsion Expertinents and Prokatypes

3. Test and Research

A. Test (No reactors curreatily in this category)

B. Research

Part N. Export Fiagctors

1. Power Reactors

A. Censtral-Station Electric Power Plands

B. Propalsion (No reactors currently in this cotegony)

2. Test, Reseanch, and Teaching
A. Gencral bradiation Test
B. Geocral Research
C. University Research and Teaching

Part V. Critical Assemblies

1. Civilitin

\section{REACTORS AND FACILT:ES \\ SHUTDOWN OR DISMANTLED}

Part I. Clvitian Reactors (Oomestlc)

1. Power Reactors

A. Centril-Station Electric Powtre Plants

B. Dual-Pupose Plants

C. Propulstion (Maritime)

2. Experimental Power-Resclot Systems

A. Electric-Power Systems

B. Space Noclear Auxiliary Power (SNAP)

C. Space Propalsion

3. Test, Research, and Universiry Reactors

A. General Iradiation Test

B. High-Power Restarth and Test

C. Safety Research and Test.

D. Goneral Research

E. University Reseanth and Teaching 


\section{CONTENTS (Continued)}

Part 日. Production Reactors

1. Materials Production

2. Process Development

Part ㅂ. Military Reactors

1. Defense Power-Reactor Applications $\quad 42$

A. Remote Installitions 42

B. Propulsion (Naval)

2. Developmental Power 45

A. Electric-Power Experingents ard Prototypes 45

B. Propulsion Experiments and Prototypes 45

3. Test and Rescarch 46
A. Test
46

B. Research

46

Part N. Export Reactors

1. Power Reactors

A. Central-Station Electric Power Plants 46

B. Propulsion $\quad 47$

2. Test, Research, and Teaching 47

A. General Iradiation Test (No reactors currentity in this category) 47

B. Gentral Researin $\quad 47$

C. Untversity Research and Teaching 47

Part V. Critteal Assemblles

1. Civilian

2.Military

48

REACTOR INDEX 


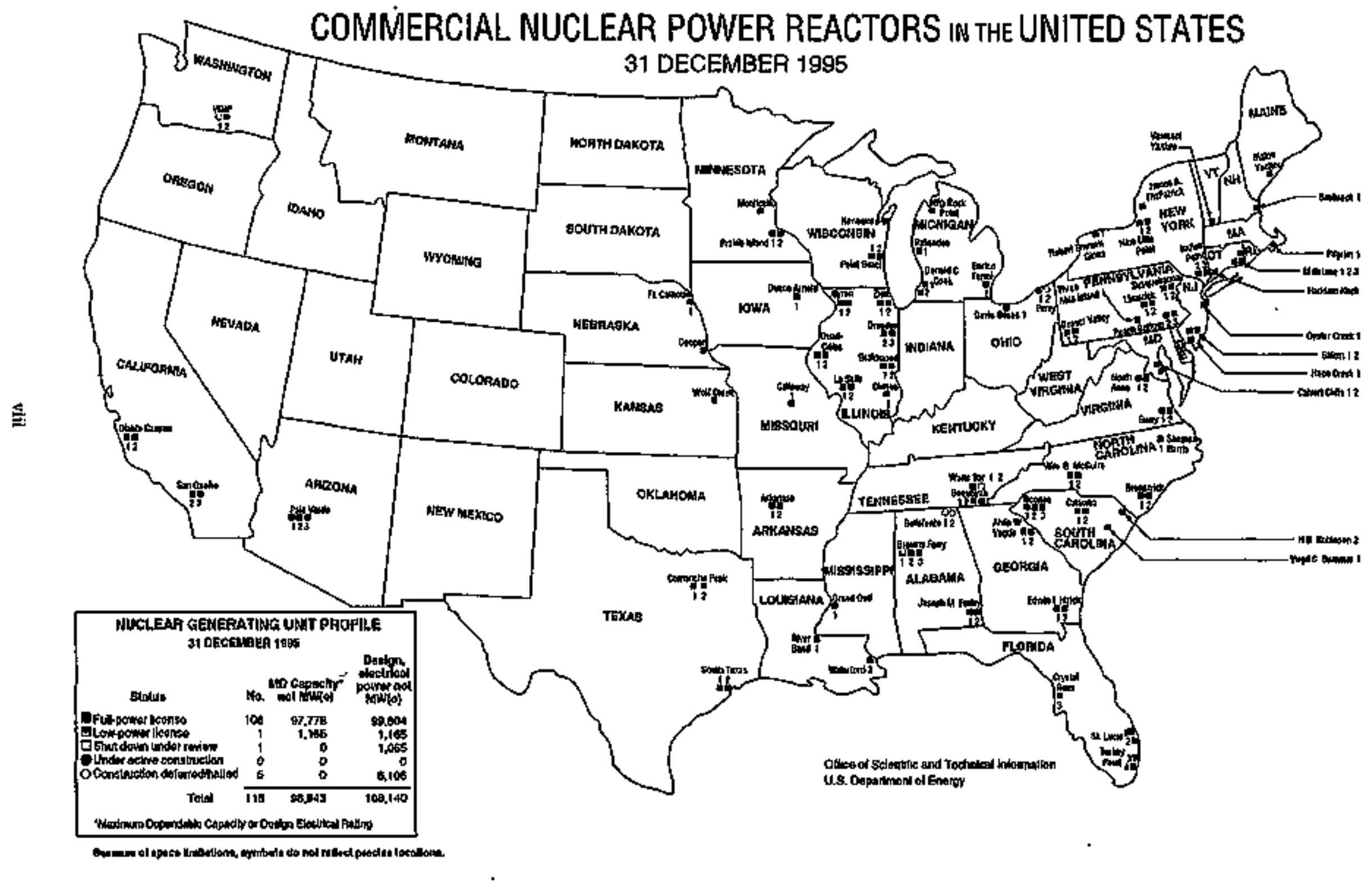


TABLE 1

COMMERCIAL NUCLEAR POWER REACTORS IN THE UNITED STATES AS OF 31 DEC 1995

\$ITE

ALABAMA

Dectiar

Decolor

Desalur

tohtan

Dothen

Socrubots

Scoitsbons

Tolnt

Arroto

Woratarg

Wintentars:

Wherstomg

Toul

ARKANSAS

Rnssellitile

Rnsectlvitio

Total

CALLFORNA

Dabblo Canyon

Dinbla Cunyen

san Ctamente

Smcinmete

Total

CONNECTHCUT

Findan Nedi:

Wuefrod

Walosoed

Wuteriond
Boome Ferry Nontes Pawer Sprion, Uail ]

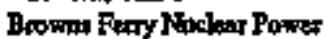
Sution, Uait 2

Bowns Rerry Moclear Power Sunfor, Unis 3

Josepd M. Fully Nodear Plant, Whail 1

Jouph ML Fulty Nocktr Plat, Unin 2

Eellefonc Nuclear Plan, Unis 1

Belleforie Noclear Plent, Unir 2

CDP

CH

SDUR

FPL $\quad 1003$

PPL $\quad 1,06$

FPL 812

62

F⿸丆

Bz

1,335

1235

3,764

7,333

Palo Vecte Nocker Geonenifig Suntion, Unit 1

Palo Vardo Nocles Generuing Solien, Ufut 2

Pato Verde Nreder Geacring Station, Unil 3

FP

FPL 1,221

1,27

FtL 1,201

1,270

FI.

1304

1,270

3,746

3,810

Aficinser Noclerone, Util 1 Aremens Nockar Ono, tinil 2

FPL

FL

\begin{tabular}{rr}
836 & 850 \\
858 & 912 \\
\hline 1,694 & 1,362
\end{tabular}

Disito Croyou Kuclear Pother Plant Uhit I

Dablo Cunyon Noctar Pown Flon, Uhi 2

Sin Onofic Nocker Geoerating Station, Unts 2

Sin Onofic Noclea Genenting Serrion, Uim 3

$\begin{array}{lll}\text { FPL } & 1,073 & 1,086 \\ \text { FHL } & 1,057 & 1,119 \\ \text { FRL } & 1,0 \% 0 & 1,050 \\ \text { FR } & 1,0980 & 1,080 \\ & 4,310 & 4,355\end{array}$

FFL $\quad 560 \quad 562$

FF1. 641 600

FPL.

873

870

FPL.

Mrilletcone Nocieter Power Station, Int 3
Faddam Neat: Pinx

Malligone Ninater Powtes Suttion. Uniti 1

Mifrina Neited Power 
TABLE 1 (Contlgued)

SIIE

FLANF NAME

CAPACTYY,

DESICN

ELECTRIChL

BATEHO

NET MT(e)

IICENSE:

STARTII

FLOBIA

Fluita $C_{15}$

Flosidin Cly

Fon Fires:

Font Piace

Red Lovel

Total

\begin{abstract}
Tnday P:in Phant Urit 3
Thikey Poim Pract, Unil 4

5 L Lucie Finer, Jakt 1

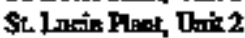

Cyyan Rivar Noctoser Ptom, Unit 3
\end{abstract}

$\begin{array}{ll}\text { FPL } & 666 \\ \text { FFL } & 666 \\ \text { IFL. } & 339 \\ \text { FPL. } & 839 \\ \text { FPL. } & 821\end{array}$

693
890
830
875

3,931

3,871

GEORGL

Bnaley

Baxigy

Whymetore

Whapesbon

Tous

‥1no1s

Britinow

Bpaiturood

Byron

Byroe

Crinton

Cordicur

Coritowa

Morrir

Monts

Sana

Seneces

Zloc:

2toc

Tote?

IOTA

Filo

Tort1

KANSAS

Burting

Tom

\section{LOUISIANA}

sc. Exatisvill.

Tith Unit 3

Toral
River Btod Sution, Uriz 1

Wuaford Gonktuing Surioo.

EJwi I. Hacti Naclear Plant, Unit 1

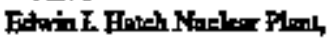
Unih 2

Alvin W. Vogle Nockar Plank, Uhis 1

Alvin W. Pogite Noslear !lant, Uais 2

FP1

FPL

FPL

FPL

T37

76

757

784

$\mathbf{1 , 1 5 8}$

1,105

2,157

1,J6h

3.809

3,362

Bridurood Stution, Unint 1

Enilonood Sation, tlimi 2

Byma Sation. Unil 2

Cincos Powt Siation, thas 1

Oratcities Sintion, Uput 1

Dresdea Nudear Poover Stution.

this 2

Dretden Nodler Power Stacion.

Unit 3

L Sello Councy Sation, Unit 1

It Sille Coonly Statiom, Unit 2

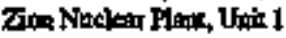

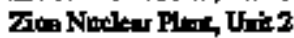

Dome Amsid Finergy Contr, Unit I

FrF

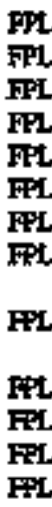

FH:

FPL

FFL

Fit

FH.

FIL

FRL.

FIL

Ft.

FFL.

FFL

\begin{tabular}{|c|c|}
\hline 1,120 & 1,120 \\
\hline 1,1220 & 1,120 \\
\hline 1,105 & 1,120 \\
\hline 1,105 & 1,120 \\
\hline 920 & 933 \\
\hline $7 \in 9$ & 788 \\
\hline $7 \%$ & 788 \\
\hline$m$ & 794 \\
\hline$m$ & 794 \\
\hline 5,0036 & 1,678 \\
\hline 3,006 & 1,078 \\
\hline 2,040 & 1,040 \\
\hline 1,040 & 1,040 \\
\hline
\end{tabular}

1,120

120

150

939

789

78

74

794

(758

1078.

1,040

1040

12,61

128t5

515

598

$515 \quad 539$

FPt.

1,134

1,170

1,134

1,170

FPL

FPL

936

936

1,075

1,104
Byron Stution, Unt 1

Qmb-Clies Suction, Udit2

Portat Powar L Litit Ca.

7210

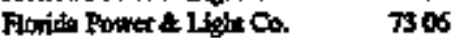

Fonitit Power e Lfotr Co. 7604

Foridn Power \& Lijit 6s, 8306

Floida Pown Comp.

TIOL

7409

780

Et 0s

$s 00$

Connompralth Ediboo Co

8705

Cunmonmetho Exisen Ca.

Commonirenth Exseon Ca

Commoninealth Ediran Co.

tilingis Power Ca

850

8701

$87 \mathrm{CP}$

Commontrell' Edison Co. 71 10

Commogrealh Edtroo Co. $\quad 7204$

Compropmenth Fitison Co. 7001

Conneorwealth Edfeos Ca $710 \mathrm{OL}$

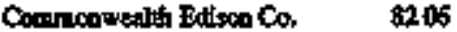

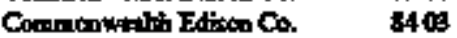

Commonwerth Edteon Co. 7306

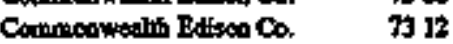

bot בecodo Light \&

Power Ca.

74 is

Wor OtedrNinder Operaing

8506 Coç.

Enexpy Oporations foc

$\$ 510$

Entery Opations fo

8503 
TABLE 1 (Continued)

SIIE

FLANTNAME

STATUS

CAPACTY,

DRSTGN

E.ECTRICAI

RATING,

NET MWU(O)

LCT

STARTUP

MArNE

Witentet

Mine Yaries Alombs Power Pinet

Totral

MLRYLAND

Lusby

Inthy

Tount

MuESACHUSETS

Pymooth

Piglint Nucleur Potwe Stution, Unis 1

Toul

Curpea Clit's Iuctear Power Pline, this:

Culvert Cifit Nuclear Pow Phen, Uinte 2

FPL

\begin{tabular}{ll}
960 & 870 \\
\hline 800 & 870
\end{tabular}

EPL

830

845

FPL

800

845

1,600

1,600

FPL

670

655

650

65

MICFIGAN

Big Roert Peint

Bridjunt

Brofyn

Newpor

South Eloven

Total

MINNESOTA

Monticoilo

Red Wing

Red wing

Toted

MISSIgsipI

Port Glosen

Totat

MISSOURI

Fuluen

Cillowsy Plent, Uutir 1

Toral

Netitsks

Browavilte

For Cillows

Gand Gulf Nuclear Stedion

PPE

\begin{tabular}{lrr} 
FPL & 67 & 72 \\
FPL & 1,000 & 1,020 \\
FPL & 1,060 & 1,090 \\
FPL & 1,085 & 1,116 \\
FPL & 730 & 805 \\
\cline { 2 - 3 } & 3,942 & 4,103
\end{tabular}

Moaticello thuclear Geoseation:

FPL

56

545

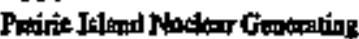

Fhnt, Uili 1

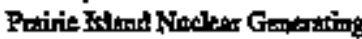

Finer, Unit2

FPL

513

530

FPL.

$\$ 12$

$\$ 30$

1,561

1.60)

1,143

1250

1,143

1250

FrL

\begin{tabular}{ll}
1,120 & 1,171 \\
\hline 5,120 & 1,171
\end{tabular}

Cooper Nibiter Sution For Cn.how Suboe, Unit 1

Fr

FIL

Tobl

\begin{tabular}{rr}
764 & 778 \\
478 & 478 \\
\hline 1,242 & 1,265
\end{tabular}

Moine Yerkec Atenis

Power Co.

$\mathbf{T 2} \mathbf{0}$

Balitmore Gas b theoule Co.

7410

Balimore Ger at Eertioc Co.

7612

Boston Etiogra Ca.

720

Conpliwer Power Ca.

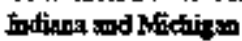

620

Eletric Co.

Inthenende Webigign

7501

白ectrisca.

TECB

Dotusil Etiogen Co.

8506

Conpromers Poner Co.

$\pi \infty$

Nibchem Sunes $F$ owat Co. 7012

Northem Stues Power Co. 7312

Horluer Sutes Poper Co. 7412

Entangy Opartions tar

8210

Urion Fiextric Co.

8410

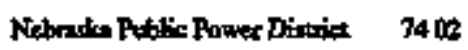
Omihe Public Poster Distica 7308 
TABLE 1 (Continued)

SIIE

NEF FAMPS়IIIRE

Sedpook

Tợnl

NET JERSEY

Salem

Silar

Salem

Toms River

Tout

NEW YORK

Buchanan

Bochenan

Ontrio

Sorito

Serdon

Soing

Total

NORTH CAROLINA

Bropl

Cowans Fond Dam

Cowtrs Ftwd Dam

Sortiport

Stroilyout

Toon

OHIO

Oll Etror

Peny

Pungy

Total

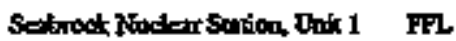

1,150

$1, \sqrt{48}$

1,150

1,148

Hope Oret Nadear Gaparies:

Srotion, Unih 1

Silen Noder Gentraing

Setion, Unh 1

Selem titudear Geoerating

Surion, Unin 2

Oymer Creck Nuctex Poner

Frast, Uan 1

\begin{tabular}{lrr} 
FPL & 1,031 & 1,061 \\
FHL & 1,106 & 1,115 \\
FHL & 1,106 & 1,115 \\
FIL & 610 & 650 \\
& & \\
\cline { 2 - 3 } & 3,853 & 3,947
\end{tabular}

Indin Point Station, Ifmit 2

FTL

$9 \$ 1$

996

Inditan Pofoc Suntion, Undis 3

PEL

Robex Emmex Gienn Nhalker

Porter Plint, Unit 1

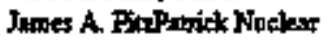

Pooper Fint

Fao Mne Patac Nueless

Siation, that 1

Nine Mile Poink Nackar

Sudor, Uhise?

FFL.

965

4 TI

965

EPL

780

470

HL $\quad \$ 6$

816

FPL

994

65

1,062

4,725

4,924

Shamon Htaml Neolear Power Hnot, Unim 1

Willism B. WeGmis Nielear Strion, Unit 1

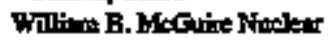

Studion, Juir 2

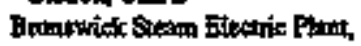
Unit 1

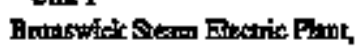
Unit2

PPL
EPL
FPL
FPL
FPL

860

900

1,120

1,580

1,129

$1,1=0$

767

825

354

821

4,63

FPL

87

FHL

1,166

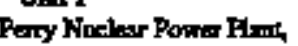

Uiti: 2

\section{Striten, Unit 1}

Pury Nirstear Power Flant
Cori
1,205
1,191

4,902

906

3,302

Condiditud Editan Co. of 73 15 Now York, he

New Yout Power Awthoily 7604

Roctieser Gos a blecurit Corp 6911

New Youk Power Ambonily $7 \$ 11$

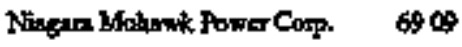

Nintura Molatws Powor Cotp. 8706

2,04

Caralina Power \& light Ca

8701

Dope Power Co,

$\$ 100$

Dole Powes C.

$\mathbf{8 4}$

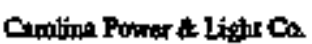

7610

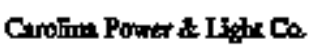

$75 \mathrm{As}$

Toledo EAson Co.

7708

conelind Elocoric

Illominsting $C a$

Cevelond Bicatic

Illominating $\mathrm{Co}$.

8606

Inder. 
FLANTNAME

caphertr,

DESTign

EEECTHEACAL

RATIVG,

STATUS NET MW(e) NET MW(e)

HCENSTE

SFARTUP

PENASYLVANIA

Bowtik

Beriolicte

Inessurt

Inenenter

Mtdaleam

Poracown

Potstown

Shifripgort

stupipiogot

Toun

sotirt CAROtnt

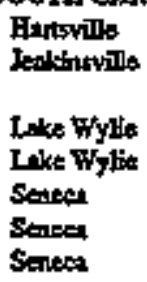

H.B. Robinsan Plang, lonit2 Vizi C. Sommer Nodkt Sunion. Unit I

Carreba Noclear Station, Unis 1 Cubwba Nudinar Striom, Unir 2 Oxones Naclear Surton, Uait 1 Oexne Nonelear Surion, Jhit 2 Doone Fuclear Sucton, Unim 3

To닌

TENNESSES

Disy

Ditsy

Spring Ciry

Spring ary

TEXAS

By cy

Bay 6ry

Otem Rase

AlmRose

VERHONT

Veman
Toml

Vermois Yanites Nudlear Rower Sution

Seruoyal Noclear hant, Linin I

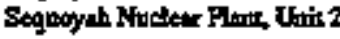

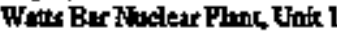
Wars Ber Nuciesr Plan, Unk 2

Sarih Tarist Powien, Unit South Teres Project, Unit 2 Comanche Peak Stoom trearile Stration, Uimit 1

Commothe Penk Suston Electic Sunion, Linin 2

Toln

Ton니

\begin{tabular}{lrr} 
FPL & 1,000 & 1,050 \\
FPL & 1,044 & 1,050 \\
FPL & 1,055 & 5,065 \\
FPL & 1,035 & 1,055 \\
FHL & 7856 & 819 \\
FPL & 1,055 & 1,055 \\
FPL & 1,055 & 1,055 \\
FPL. & 810 & 835 \\
FPL & 820 & 836 \\
& & 8,830 \\
\cline { 2 - 3 } & 8,700 &
\end{tabular}

\begin{tabular}{lrr} 
FPL & 663 & 700 \\
FPL & 885 & 900 \\
FPL & 1,129 & 1,145 \\
FPL. & 1,129 & 1,145 \\
FRL & 846 & 886 \\
FPL & 846 & 886 \\
FPL & 846 & 816 \\
\cline { 2 - 3 } & 6,364 & 6548
\end{tabular}

\begin{tabular}{lll} 
FFL & 1,122 & 1,148 \\
FPL & 1,122 & 1,145 \\
LPL & 1,165 & 1,165 \\
COH & & 1,165 \\
\cline { 2 - 3 } & & \\
\cline { 2 - 3 } & &
\end{tabular}

Tennesse Valleg Aurdority

Tomeses Vallo Amtiontity

Temerter Valley Aubority

Tennesce Valley ubthotiy

sot

81 i1

9511

Indef.

8219

8405

70

340

1406

412

8908

7606

8708

7009

801

8605

704

7311

740

\begin{tabular}{|c|c|c|c|c|}
\hline $\begin{array}{l}\text { FPL } \\
\text { FPL } \\
\text { FPL }\end{array}$ & $\begin{array}{l}1,251 \\
1,251 \\
1,150\end{array}$ & $\begin{array}{l}1,251 \\
1,251 \\
1,150\end{array}$ & 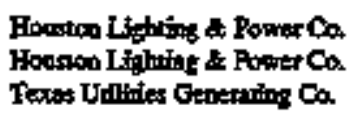 & $\begin{array}{l}8805 \\
5902 \\
9004\end{array}$ \\
\hline FP. & 1,150 & 1,150 & Teus Utibities Genprating $\mathrm{Co}$. & 93 \\
\hline
\end{tabular}

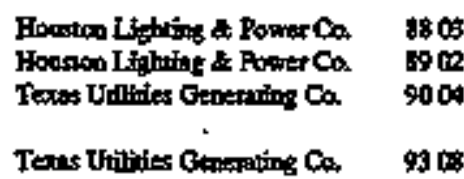

$4,802 \quad 4,800$

FPL 504

514 
TABLE 1 (Continued)

SITE

PLAMT NAME

Sury Power Striben, Und 1 Sung Poner Station, thí12

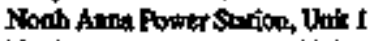

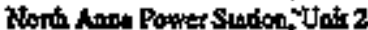

Tocal

Wastingtion Noolese Project, Unir 1

Richluad

Sichlind

Washingan Noclest Frojea, Unir 2

CDA

\begin{tabular}{ll}
1,266 \\
1,086 & 1,100 \\
\hline 1,086 & 2,346
\end{tabular}

Tots

Kewnonse Nucier Powes Punt

Potint Beach Nockear Flint, Dnil J

Poine Beach Nuctedr Pince.

FHL

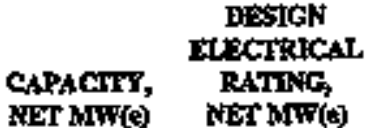

LCXNSSDE

STHRTUP

\begin{tabular}{lrr} 
FWL & 781 & 788 \\
FPL & 791 & 788 \\
FPL & 900 & 907 \\
FPL & 867 & 909 \\
\cline { 2 - 3 } & 3399 & 3390
\end{tabular}

Vizipia Elextrica Power Ca. Virjinio Electric \& Powt Ca. Vyptnla Electric \& Fow $\mathrm{C}_{0}$.

720

73 助

7804

Virginia Eletic \& Pown Co. $\quad 8006$

WISCONSIN

\section{Cotion}

Two Cresks

Two Coust

$$
\text { Untit } 2 .
$$

Totol

U.S. Total

PRL, Fath Fowar Liesane

IPl, 10w-Power lictote

UC, Dodor Acdive Constroxtico

\begin{tabular}{lrr} 
FRL & 511 & 535 \\
FPL & 485 & 457 \\
FPL & 435 & 497 \\
& & \\
\hline 1,481 & 1,529 \\
\hline 98,943 & $j(198,140$
\end{tabular}

CDH, Condruation Deferteditisted SDUR, Star Down Under Reriers 
TAELE 2

\section{STATISTICAL SUMMARY OF NUCLEAR REACTORS}

\section{AS OF 31 DEC 1995}

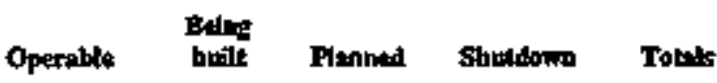

\section{US. REACTORS}

CIVILAN RXACTORS (DOMESTIC)

Purtar Beoctions

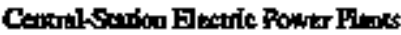

Durt-Porpose Hrots

Prepolition (Afotime)

109

5

Frogimestal Pewer-Reector Sjstems

Dextric-Power Synterrs

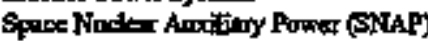

Spare Rropolinim (Rowis)

Test, Hesearch, and Ltalessty Resters Gencell Intimion Test

High-Powe Reasech and Tet

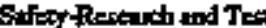

Generil Resent

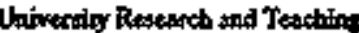

PROBUCTION REACTORS

Moturits Production

Process Developinent

MILITARY REACTORS

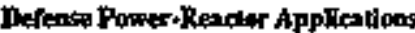

Remote Installations

Prosetlition (Novil)

Deretepnepal Power

Electrie-Power Experimants and Protoryes: Propulion Experiments and Prolotypes

Tot not Rewarth

Teat

Revestich

$\begin{array}{rr}20 & 134 \\ 1 & 1 \\ 1 & 1 \\ 24 & 24 \\ 5 & 9 \\ 21 & 21 \\ 6 & 7 \\ 8 & 12 \\ 10 & 10 \\ 57 & 71 \\ 31 & 6 \\ 12 & 13 \\ 5 & 5\end{array}$

112

4

66

1

14

34

!

4

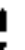

4

9

12

6

13

5

\section{EXPORT REACTORS}

\section{POWER REACTORS}

Centraistutian Frectrit Ponter Pints

Propulston

TEST, RESTARCH, AND TEACFING

Genersil Iroditation Test

General Revearch

Untrersikg Rouret and Teabing

Toks

st

$\begin{array}{r}\frac{7}{27} \\ 19 \\ \hline 305\end{array}$
8

8

18
6

$7 \quad 7$

4

tid

625

$356 \quad \overline{769}$ 
TABLE 3

\section{AGEREVIATIONS OF CONTAACTOAS, DESIGNERS, BHIPBULDERB, AND FACILITY OPERATOAS}

Thls list conteins references to cutreat and historical corporale ant government strukture.

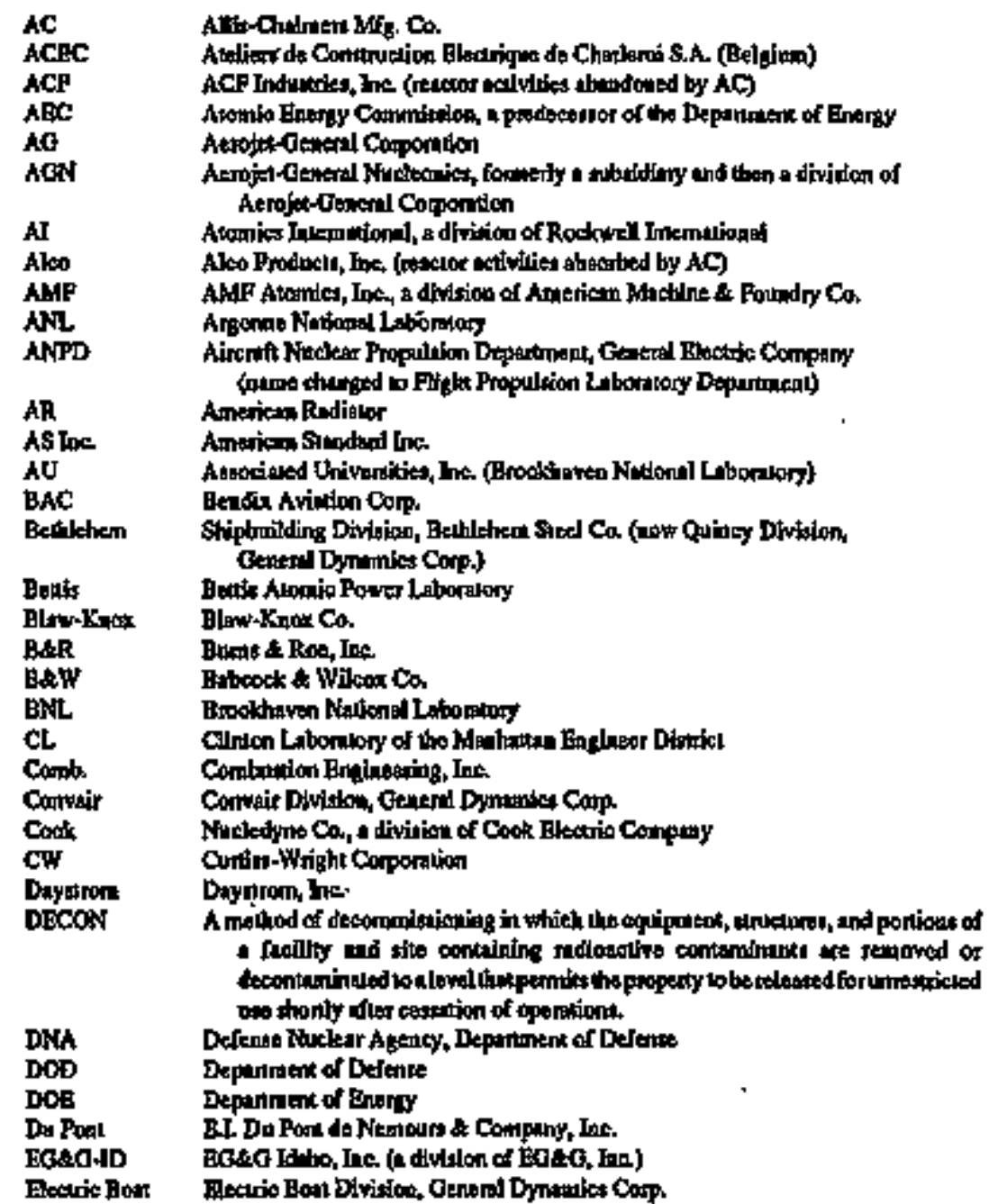

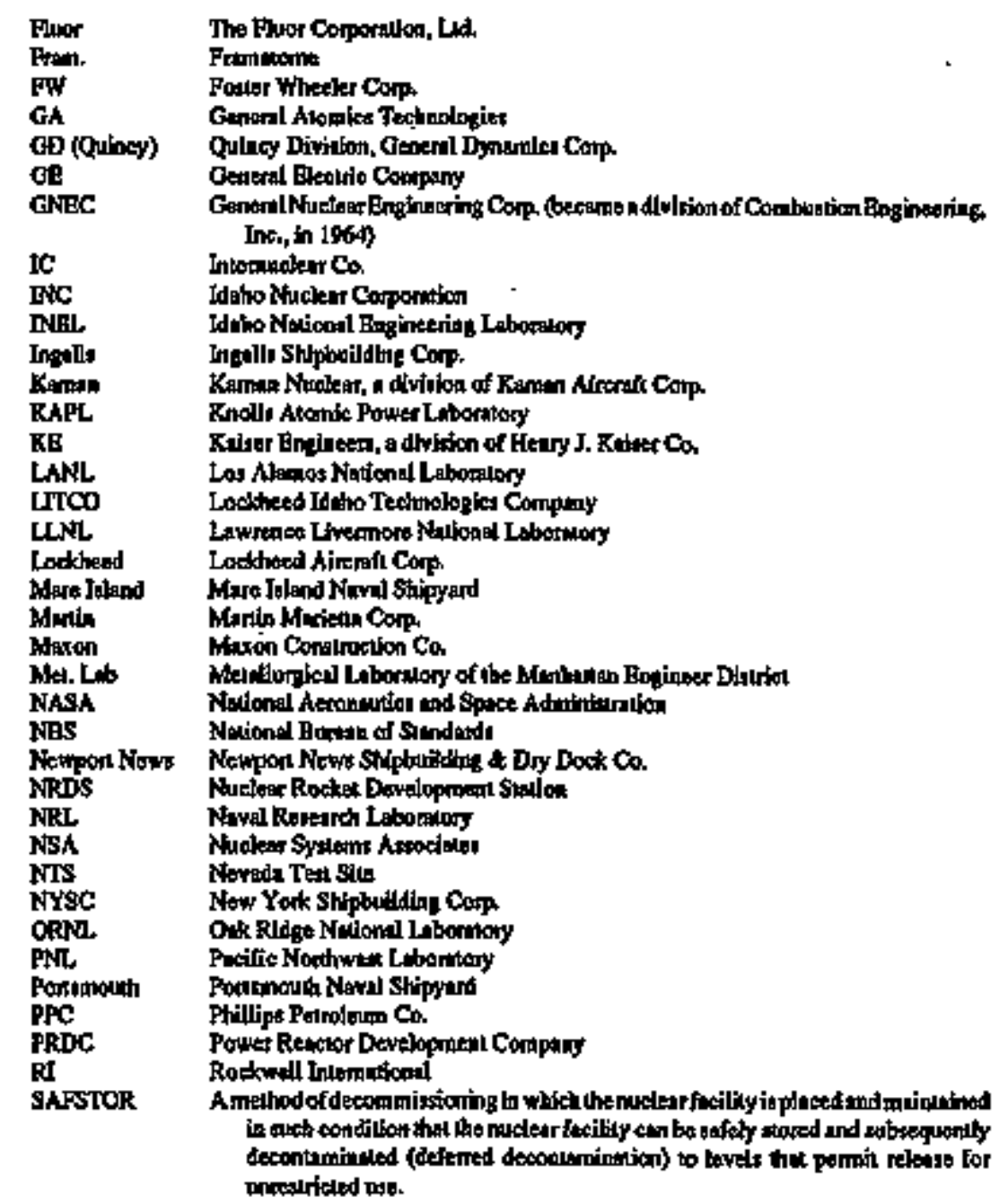




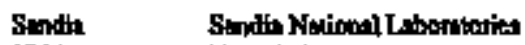

UNCC Unhed Nucles Corpostion, Developinent Divition

Vins Viro Corponition of Americe

Wor. Westinghooses Elatrite Corpontion

WHC Wealinghooss Hutrond $C_{0}$. 


\section{REACTORS AND FACILITIES OPERABLE, BEING BUILT, OR PLANNED}




\section{POWER REACTORS}

\section{A. Central-Station Electrle Power Ptants}

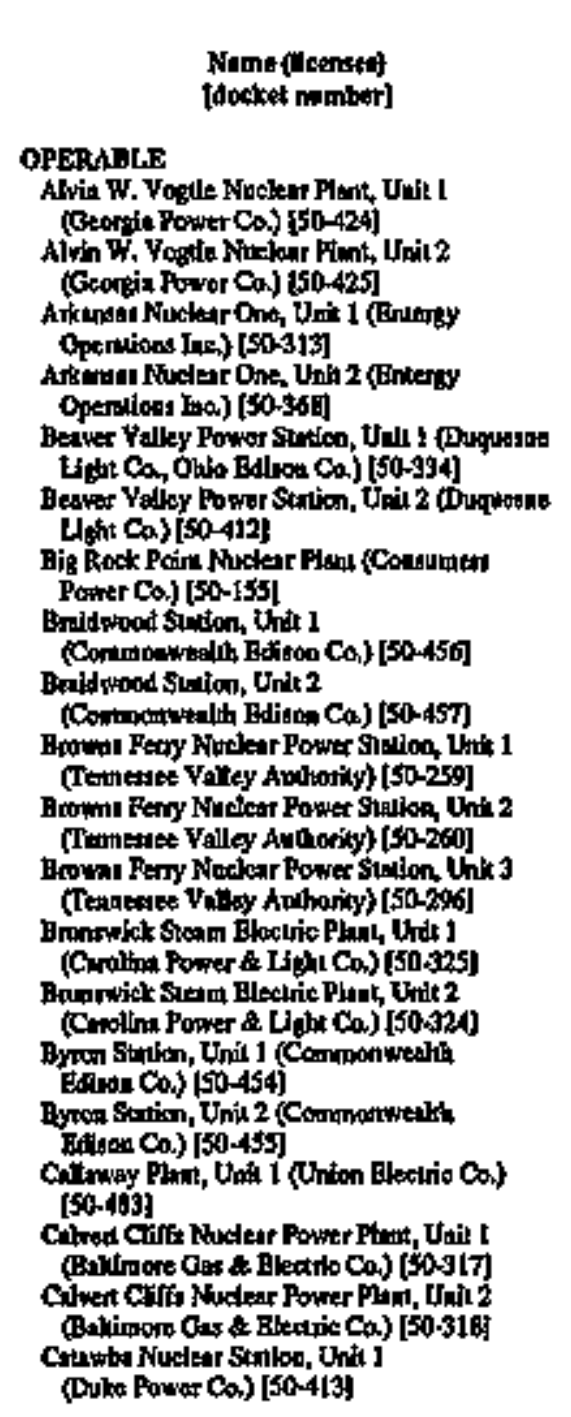

\begin{tabular}{|c|c|c|c|c|c|}
\hline Lecalion & 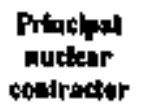 & Type & 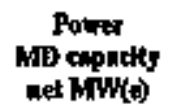 & $\begin{array}{l}\text { Power } \\
\text { Lensed } \\
\text { MW(0) }\end{array}$ & $\begin{array}{c}\text { Inllinal } \\
\text { critkenllity } \\
\text { (yr mot) }\end{array}$ \\
\hline Waresbar, GA & Wost. & Pressurized & 1158.0 & 3965,0 & 8705 \\
\hline Wifyretboro, GA & Fet!. & 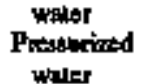 & 157.0 & 3565.0 & 1903 \\
\hline Rposelbilld, AR & ERW & Presbatized & 836.0 & 2568.0 & 7408 \\
\hline Ruscellyilit, AR & Comb. & Piesurioged & 858.0 & 2815.0 & 7812 \\
\hline Shtppinepan, PA & Went. & $\begin{array}{l}\text { Pranourized } \\
\text { ralos }\end{array}$ & 8100 & 2652,0 & $360 s$ \\
\hline Shippinepori, PA & Weal. & $\begin{array}{l}\text { Prestanized } \\
\text { walcr }\end{array}$ & 820.0 & 2653.0 & 9708 \\
\hline Bls Rock Potnl, bat & $\mathbf{G E}$ & Boollne walor & 67.0 & 2400 & 6209 \\
\hline Bniduroed, Il & Wes!. & $\begin{array}{c}\text { Prsowpized } \\
\text { walor }\end{array}$ & $1+2000$ & 3411.0 & 1706 \\
\hline Brtidurood, I & wort. & $\begin{array}{c}\text { Pressurized } \\
\text { waler }\end{array}$ & $\operatorname{l1200} 0$ & 3411,0 & $\mathrm{BBO3}$ \\
\hline Docalur, AL & OE & Boting water & 0.0 & 3293.0 & 7304 \\
\hline Decotion, A. A. & CE & Bołlng waler & 10650 & 3293.0 & 7407 \\
\hline Decatiur, Al & an & Boiting witer & 1065,0 & 3293,0 & 3609 \\
\hline Soutipon, NC & as & Boulling waser & 767.0 & 2496.0 & 7610 \\
\hline Sounhpon, NC & $\mathrm{ot}$ & Bolllnt water & 754,0 & 2430.0 & 7500 \\
\hline Byron, IL & Wo1 & Presuchiogd & $\tan \theta$ & 3411.0 & 1502 \\
\hline Bymon, $\pi$ & Wien. & Predustred & ISOSA & 3411.0 & ET 01 \\
\hline Pullon, No & Wtat. & Presturiod & 1120.0 & 3565.0 & 6410 \\
\hline Luby, MDD & Comb. & $\begin{array}{c}\text { Peanuribed } \\
\text { Waler }\end{array}$ & 830.0 & 2700.0 & 7410 \\
\hline Lasby, MD & Canbs & $\begin{array}{c}\text { Prescurized } \\
\text { waler }\end{array}$ & 800 & 2700.0 & 7611 \\
\hline LNe Wylie, SC & Wast & $\begin{array}{l}\text { Proanurized } \\
\text { waler }\end{array}$ & 1129.0 & 3411.0 & 0501 \\
\hline
\end{tabular}

\section{PART I CIVILIAN REACTORS (DOMESTIC)}

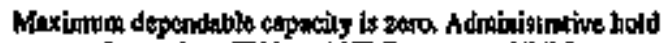

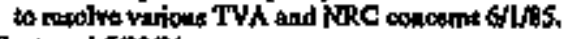
Restanted 5pav9l.

Rentarted 11/19/95.
Comment

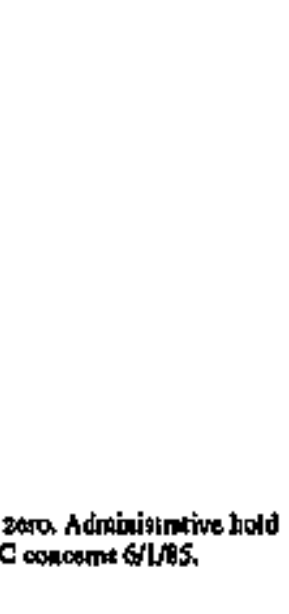




\begin{tabular}{|c|c|c|c|c|c|c|}
\hline 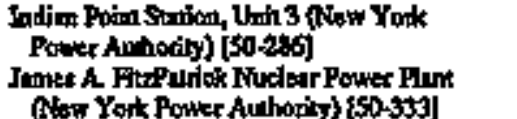 & $\begin{array}{l}\text { Bachanon, NY } \\
\text { Sciba, NY }\end{array}$ & Went. & 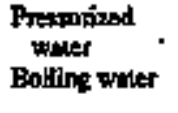 & 7800 & 2436,0 & 74 it \\
\hline 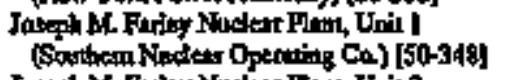 & Doxllom, AL & Wort. & $\begin{array}{l}\text { Poxtamizized } \\
\text { vouter }\end{array}$ & 8120 & 2652.0 & 708 \\
\hline 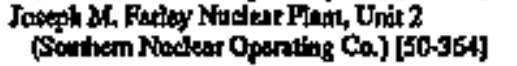 & Dodhm, Al & Went. & $\begin{array}{l}\text { Prosplnimed } \\
\text { water }\end{array}$ & 8220 & 2652.0 & 81 cos \\
\hline 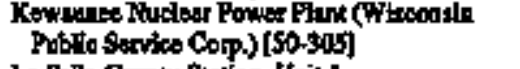 & Oritan, HI & Went. & $\begin{array}{l}\text { Presurized } \\
\text { wart }\end{array}$ & 511.0 & 1650.0 & 740 \\
\hline $\begin{array}{l}\text { 1. Stge Cointy Stalion, Unil } 1 \\
\text { (Commonwealh Bdition Ca) [50-37s] }\end{array}$ & steres, II & GE & Boling watter & 1036.0 & 3323.0 & 8206 \\
\hline 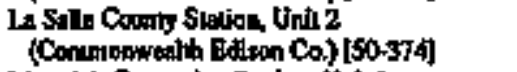 & Sexeck, II. & $\mathbf{G B}$ & Boitng water & I0060 & 3393.0 & 840 \\
\hline 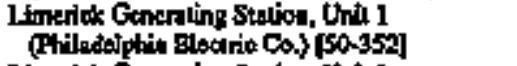 & Podt:How1, PA & $\mathbf{G B}$ & Boiling waiks & 10550 & 3293.0 & 8412 \\
\hline 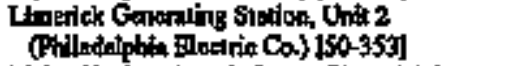 & Fotstow, PA & GE & Boilings wator & 1055.0 & 3293.0 & 5900 \\
\hline 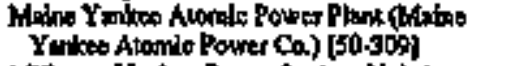 & Wincussel, ME & Conth & $\begin{array}{l}\text { Prapurizod } \\
\text { mater }\end{array}$ & 866,0 & 27000 & 7210 \\
\hline 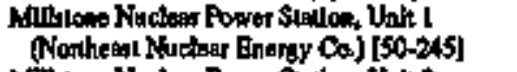 & Waleftond, CT & GE & Bolling wathor & 641.0 & 2031.0 & 7010 \\
\hline 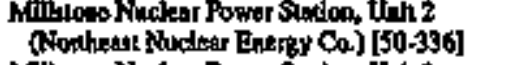 & Walerfond, CT & Conb. & $\begin{array}{l}\text { Pritgurizod } \\
\text { twitrer }\end{array}$ & 979.0 & 2700.0 & 7510 \\
\hline 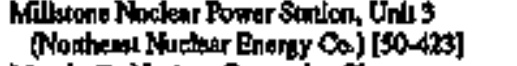 & Wheriond, CI' & Wesl. & $\begin{array}{l}\text { Prosenuized } \\
\text { wraler }\end{array}$ & 11370 & 3911.0 & 8601 \\
\hline 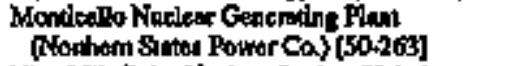 & Moalkello, MN & OE & Bating waler & 5960 & 1670.0 & 7012 \\
\hline 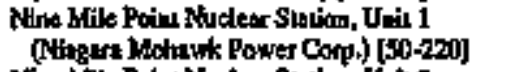 & Scribu, NY & GB & Bodling wmer & 5650 & 2150.0 & 6909 \\
\hline 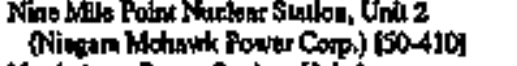 & Scrlbu, $\mathrm{NY}$ & $\mathrm{OB}$ & Bolting whior & 9940 & 33230 & 8705 \\
\hline 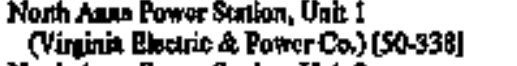 & Mlated, VA & Wox. & Praspinized & 9000 & 2895,0 & 7604 \\
\hline 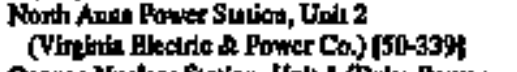 & Afineral, VA & Wex. & $\begin{array}{l}\text { Prostunized } \\
\text { water. }\end{array}$ & 867.0 & 2893.0 & 8006 \\
\hline 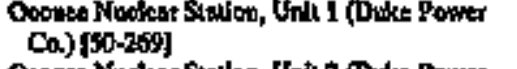 & Serech, 50 & B\&W & $\begin{array}{l}\text { Prasurital } \\
\text { water. }\end{array}$ & 8460 & 2568.0 & 7304 \\
\hline $\begin{array}{l}\text { Oconse Nuctaur Stallon, Unil } 2 \text { (Driks Power } \\
\text { Ca.) [50-270] }\end{array}$ & $S=1000,3 C$ & BSW & $\begin{array}{l}\text { Prospunized } \\
\text { thintox }\end{array}$ & 8460 & 25668.0 & 7311 \\
\hline 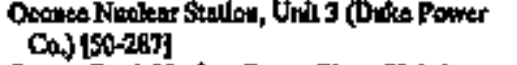 & Strect, $s c t$ & BdW & $\begin{array}{l}\text { Prossurizout } \\
\text { water }\end{array}$ & 8460 & 2569.0 & 7409 \\
\hline 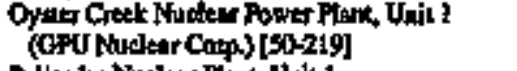 & Totha River, NO & OH & Bolling waler & 610.0 & 1930.0 & 6905 \\
\hline Pilinute Nuckes Plonk, Onil1 1 & Scouh Howen, $\mathrm{WJ}$ & Conbl. & $\begin{array}{l}\text { Poulumitud } \\
\text { Walot }\end{array}$ & 730,0 & 25300 & T10s \\
\hline 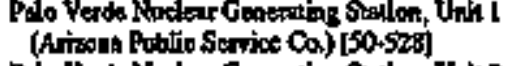 & Wintestours $A Z$ & Conp. & $\begin{array}{l}\text { Preituilzed } \\
\text { wuter }\end{array}$ & 1221.0 & 3800,0 & 8503 \\
\hline 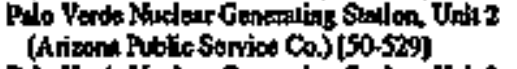 & Wropersbung, AZ & $C_{0, p b}$ & $\begin{array}{l}\text { Preanurbed } \\
\text { waler }\end{array}$ & 12210 & 35000 & 1604 \\
\hline 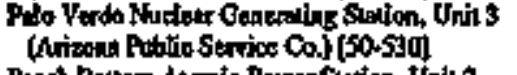 & Wintorabwere AZ & Corth. & Poesubitiod & 1904.0 & 3517.0 & 6710 \\
\hline 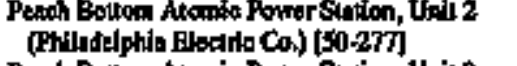 & Lencadter, Ph & Gt & Bet.'ing water & 1085.0 & 3293,0 & 7309 \\
\hline 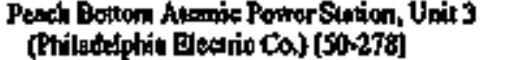 & Lancauter, Ph & OE & Bdt"lis water & 1035.0 & 3298.0 & 408 \\
\hline
\end{tabular}




\section{POWER REACTORS}

\section{A. Conlra-Staton Electric Power Plants (Continued)}

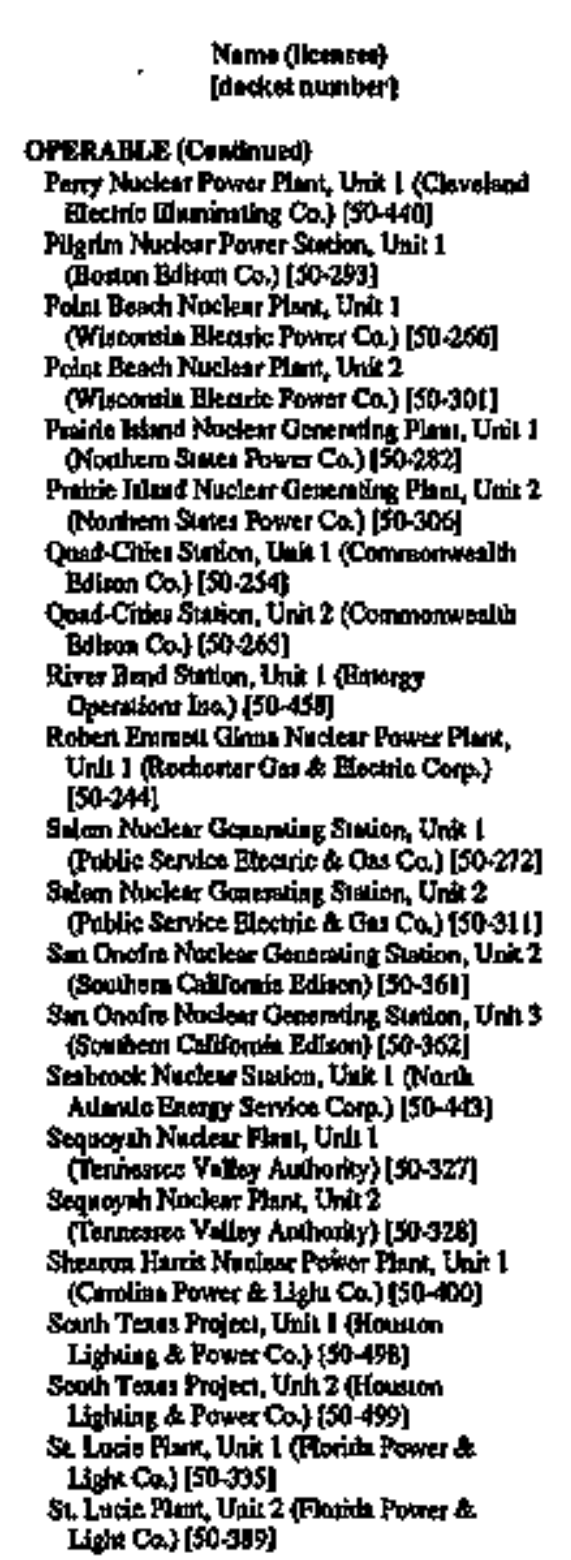

\section{PART I CIVILIAN FEACTOFS (DONESTIC)}

\begin{tabular}{|c|c|c|c|c|c|}
\hline Lometlion & $\begin{array}{c}\text { Prindpal } \\
\text { muclasar } \\
\text { contrinter }\end{array}$ & $\mathrm{T}_{\mathrm{spe}}$ & 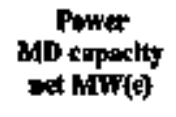 & $\begin{array}{l}\text { Ponser } \\
\text { Lkespod } \\
\text { MW(0) }\end{array}$ & 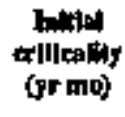 \\
\hline Pang, OH & $\mathrm{GB}$ & Boliting water & 11660 & 25790 & 8606 \\
\hline Flymont, MA & GH & Bofing waler & 670.8 & 1991.0 & 7206 \\
\hline Two Croekt, WI & Weth & Premantesed & 485.0 & 1519.0 & 7011 \\
\hline Two Crtaks, W & Woss. & Pratinized & 4850 & 151920 & 3205 \\
\hline Red Wrag, $M N$ & Went. & Preturized & 513.0 & 16500 & 7312 \\
\hline Red Wing, MN & Wes. & $\begin{array}{l}\text { Pressulized } \\
\text { proter }\end{array}$ & 5120 & 1650.0 & 7412 \\
\hline Condove, I & $\mathbf{G B}$ & Boling uner & 769.0 & 2511.0 & 7110 \\
\hline Condorm, IL. & GE & Hoiling waler & 769.0 & 2911.0 & 7204 \\
\hline St, Frendintly, LA & GE & Boiling water & 936, & 28940 & 6510 \\
\hline Outudo, NY & Went. & Pressunized & 470.5 & 1 squo & 911 \\
\hline Salen Nu & Won. & $\underset{\text { Presturized }}{\text { Paut }}$ & 10060 & 3411.0 & 7612 \\
\hline Sulant $N$ & Won. & $\begin{array}{l}\text { Propofurized } \\
\text { wavet }\end{array}$ & 10060 & $\$ 411.0$ & 5008 \\
\hline Stre Ckitende, CA & Conth & $\begin{array}{l}\text { Ptesturized } \\
\text { walor }\end{array}$ & to70.0 & 3390.0 & 207 \\
\hline San Clonante, CA & Comb. & $\begin{array}{l}\text { Presubriped } \\
\text { maver }\end{array}$ & 1090,0 & 9390,0 & 8300 \\
\hline Sasbrook, NH & Went. & $\begin{array}{l}\text { Preimuilzed } \\
\text { wales }\end{array}$ & 1350.0 & 3411.0 & 6906 \\
\hline Dalis, TN & Wesl. & $\begin{array}{c}\text { Pegiumbed } \\
\text { water }\end{array}$ & 1122.0 & $34 t 1,0$ & 1000 \\
\hline Daing. T & Worl. & Pressmiased & 1122.0 & 34110 & $81 \mathrm{El}$ \\
\hline Bonat: & Wetl. & $\begin{array}{l}\text { Powsucintod } \\
\text { waler }\end{array}$ & 860.0 & $2 \pi 75.0$ & 7701 \\
\hline Bay 0 & Whet & $\begin{array}{c}\text { Pressodized } \\
\text { water }\end{array}$ & 1251.0 & 3800.0 & 8803 \\
\hline Bay C & Wost. & $\begin{array}{l}\text { Presnationd } \\
\text { waver. }\end{array}$ & 1251.0 & 98000 & 900 \\
\hline F & $C_{0}=$ & $\begin{array}{c}\text { Prostuined } \\
\text { water }\end{array}$ & Bas.0 & 2700.0 & 7604 \\
\hline $\mathbf{P a t}$ & Comb. & $\begin{array}{l}\text { Prusondzad } \\
\text { uniter }\end{array}$ & 839.0 & 27000 & 8306 \\
\hline
\end{tabular}

Comment 


\begin{tabular}{|c|c|c|c|c|c|c|}
\hline 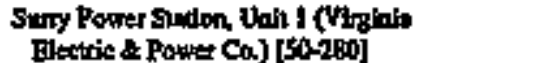 & GravelNeck, VA & Weal. & $\begin{array}{l}\text { Pressulitied } \\
\text { writer }\end{array}$ & 781.0 & 2441.0 & $70 r$ \\
\hline 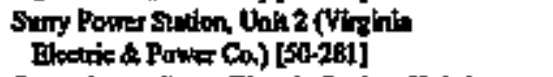 & Grnvel Neck, VA & Wust. & $\begin{array}{l}\text { Prestutiaced } \\
\text { woter }\end{array}$ & T81.0 & 2441.10 & 7908 \\
\hline 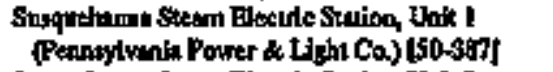 & Barwick, PA & $\mathbf{C B}$ & Boalign weler & 10400 & 3293.0 & 820 \\
\hline 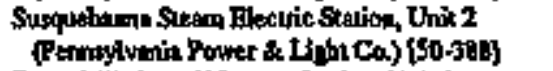 & Berwick, PA & $\mathrm{GE}$ & Boiling wuer & 10440 & 5263.0 & 8405 \\
\hline 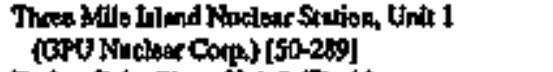 & Mlddesom, $\mathbf{P A}$ & B. $\mathbf{W}$ & $\begin{array}{l}\text { Preandizyed } \\
\text { whiter }\end{array}$ & 7850 & 2568.0 & 7406 \\
\hline 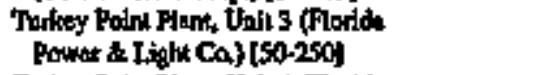 & Floridn Cizy, F & Prat. & $\begin{array}{l}\text { Prethurized } \\
\text { walex }\end{array}$ & 665.0 & 22000 & 7210 \\
\hline 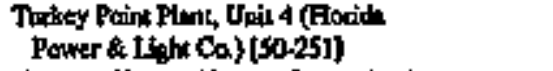 & Florida City. Ft & Wes:. & $\begin{array}{l}\text { Prossuritred } \\
\text { water }\end{array}$ & 606 & 2200.0 & 7306 \\
\hline 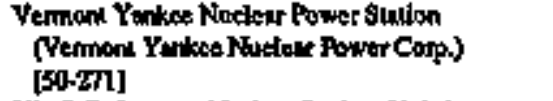 & Venton, VT & as & Boilfag waver & SOA. 0 & 1593.0 & 7263 \\
\hline 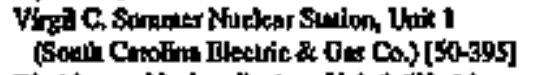 & Janktianvills, SC & Presl. & $\begin{array}{l}\text { Presurized } \\
\text { waber }\end{array}$ & 8BS.0 & 27750 & 8210 \\
\hline 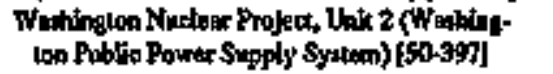 & Btscalend, Wh & $\mathrm{CE}$ & Bodling wat & 1066.0 & $33 \mathbf{2 3}, 0$ & 8401 \\
\hline 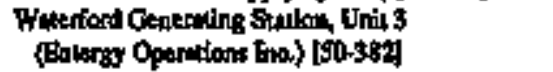 & Tafi, LA & Comits. & 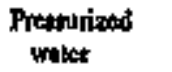 & $\cos 50$ & 33900 & 8503 \\
\hline 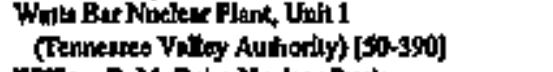 & Spring Cily, TN & Wost. & $\begin{array}{l}\text { Prishuritzed } \\
\text { water. }\end{array}$ & $\$ 165.0$ & $34 t 5+0$ & $95 \mathrm{JI}$ \\
\hline 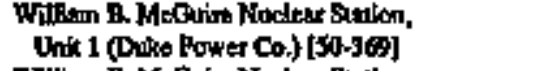 & $\begin{array}{l}\text { Cownul Ford D*n, } \\
\text { NC }\end{array}$ & Wesl. & $\begin{array}{l}\text { Preaririasod } \\
\text { wake: }\end{array}$ & 11290 & $\$ 411.0$ & 8108 \\
\hline 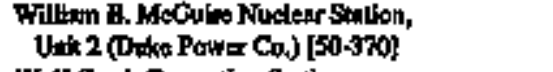 & Conna Fond Dam, & Wosl. & $\begin{array}{l}\text { Pretututized } \\
\text { mallor }\end{array}$ & d I & $34 t 1.0$ & 8305 \\
\hline 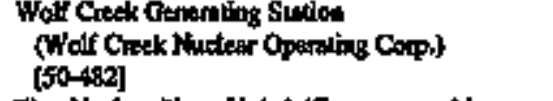 & Ahrlingtom, KS & Wos. & $\begin{array}{l}\text { Presturitued } \\
\text { meter }\end{array}$ & 134.0 & 3555.0 & 8505 \\
\hline 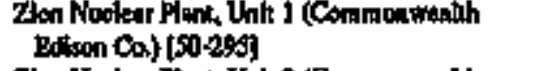 & Z̃ot, $\mathbb{U}$ & Teal. & $\begin{array}{l}\text { Prennuized } \\
\text { walot }\end{array}$ & 10900 & 3750.0 & 7306 \\
\hline 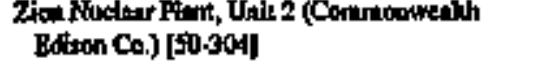 & Zzon, IL. & Wan. & $\begin{array}{l}\text { Prodeuritzod } \\
\text { watee }\end{array}$ & 104000 & 3250,0 & $73 \mathbf{~} 2$ \\
\hline $\begin{array}{l}\text { Narue (keanses) } \\
\text { [decket muniber] }\end{array}$ & Lotalke & 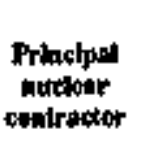 & $\mathrm{Ty}$ & 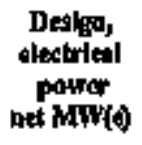 & $\begin{array}{c}\text { Desdgn, } \\
\text { partor } \\
\text { Mtwer }\end{array}$ & $\begin{array}{l}\text { Bsilmated } \\
\text { Iafilal } \\
\text { cilleallity } \\
\text { (jT mo' }\end{array}$ \\
\hline \multicolumn{7}{|l|}{ BXNG DUILT } \\
\hline 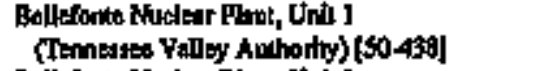 & Solutibora, AL & Bes $\mathbf{W}$ & $\begin{array}{l}\text { Preaturizod } \\
\text { walke: }\end{array}$ & 1235.0 & 3760.0 & Indef. \\
\hline 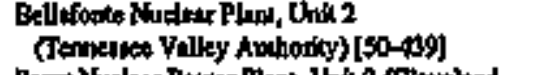 & Spollobotb, AL & BAw & $\begin{array}{l}\text { Prothinined } \\
\text { water }\end{array}$ & 1235.0 & 37600 & Indef. \\
\hline 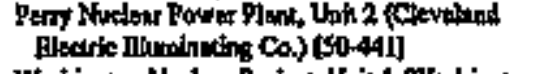 & Pony, 애 & GB & Eojling waltir & 1205.8 & 3759.0 & Inded. \\
\hline $\begin{array}{l}\text { Whatington Nueloar Project, Unit I (Wophingtion } \\
\text { Publlo Power Supply Syumen) [50-460] }\end{array}$ & Rkhlend, WA & BetW & $\begin{array}{l}\text { Poranurizad } \\
\text { onter }\end{array}$ & 12660 & 376010 & Indet. \\
\hline 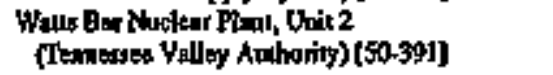 & Spring Chy. IN & Wogl. & $\begin{array}{l}\text { Pressurized } \\
\text { waker }\end{array}$ & 1165.0 & 3411,0 & inder. \\
\hline
\end{tabular}

Recolved low power operating llcense on ILSWs. Recrived foll powar operiling liesnac on 2ht/66. 
B. Dual-Purpose Plants

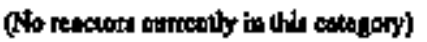

C. Propulgion (Marithme)

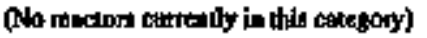

\section{EXPEFIMENTAL POWER-REACTOR SYSTEMS}

A. Electrlo-Power Systems

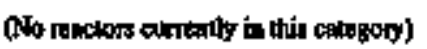

B. Space Nublear Auxillary Power (SNAP)

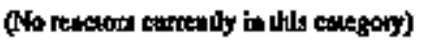

C. Spape Propulsion

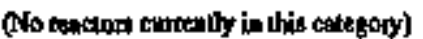

\section{TEST, RESEARCH, AND UNIVERSITY REACTORS}

A. Generat Irradlation Test

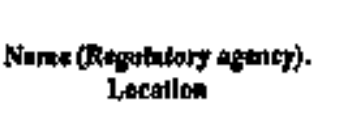

OFERADL

Atransed Tool Heactor (CO6)

NEL, ID

\begin{tabular}{|c|c|c|c|c|c|}
\hline $\begin{array}{l}\text { Rower } \\
\text { espatty } \\
\text { net } k F(e)\end{array}$ & $\begin{array}{l}\text { Power } \\
\text { foensed } \\
\text { KWW(0 }\end{array}$ & $\begin{array}{l}\text { Aultiarlized } \\
\text { power } \\
\text { kW(t) }\end{array}$ & $\begin{array}{c}\text { Pawer } \\
\text { MD capactly } \\
\text { net MWW(t) }\end{array}$ & $\begin{array}{l}\text { Power } \\
\text { licensed } \\
\text { MWt(T) }\end{array}$ & $\begin{array}{c}\text { Infiltal } \\
\text { crikkality } \\
\text { (yrmo) }\end{array}$ \\
\hline
\end{tabular}

Combent
$250,000.0$
6800 ATR, Tank

ATR. Tank
LTCO
Openting. 


\section{B. Hilgh-Power Research and Test}

\begin{tabular}{|c|c|c|c|c|c|c|c|c|}
\hline Name (Regalintory agencs). & $\begin{array}{l}\text { Powny } \\
\text { capedily } \\
\text { netkin(g) }\end{array}$ & $\begin{array}{l}\text { Power } \\
\text { Ureensed } \\
\text { kwoto }\end{array}$ & $\begin{array}{l}\text { Aushorlyed } \\
\text { poiner } \\
\text { kWr(t) }\end{array}$ & 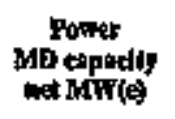 & 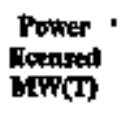 & $\begin{array}{l}\text { Inltial } \\
\text { cr:Ulendlty } \\
\text { (grmo) }\end{array}$ & 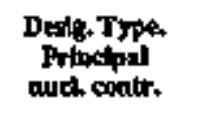 & Cerninent \\
\hline 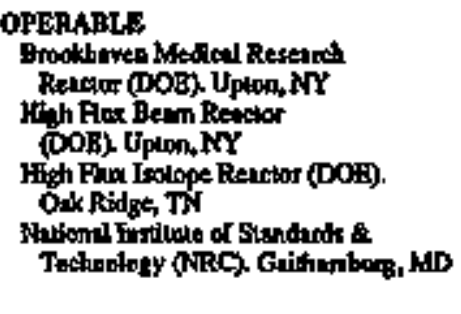 & & & $\begin{array}{r}3,00000 \\
30,00000 \\
85,000,0 \\
20,000,0\end{array}$ & & & $\begin{array}{l}5900 \\
6510 \\
6500 \\
6700\end{array}$ & 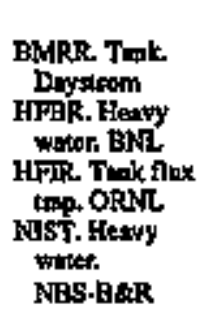 & 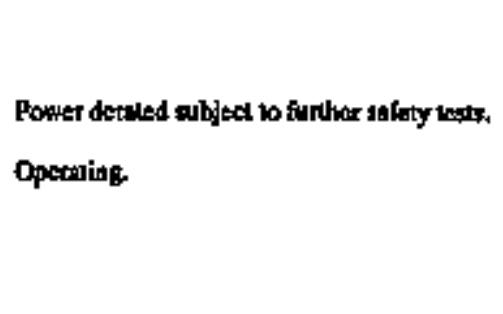 \\
\hline
\end{tabular}

\section{Safety Research and Test}

(Nip roution currently in thit chofory)

\section{General Research}

\begin{tabular}{|c|c|c|c|c|c|}
\hline \multicolumn{6}{|l|}{ OPERABCE } \\
\hline 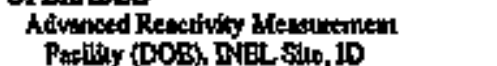 & 100.0 & & & $\begin{array}{l}\text { ARHF, Pool } \\
\text { ITTCO }\end{array}$ & 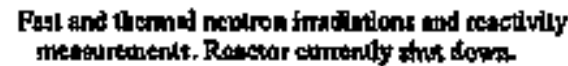 \\
\hline $\begin{array}{l}\text { Aopletit Operations, Inc. (NRC). } \\
\text { Sin Renom, CA }\end{array}$ & 250.0 & & 6500 & $\begin{array}{l}\text { AGTNR. } \\
\text { Pow.TRKGA } \\
\text { cors GA }\end{array}$ & \\
\hline 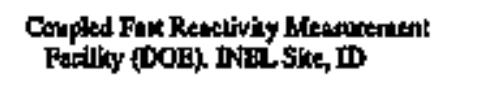 & 1000 & & 6100 & $\begin{array}{l}\text { CPRHF. Pool. } \\
\text { LTTCO }\end{array}$ & 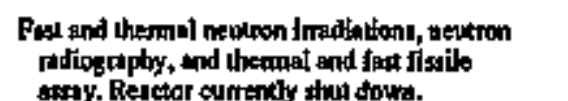 \\
\hline Daw Cimioul Co. (NRC). Mildend, MI & 300,0 & & 6700 & 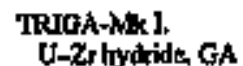 & \\
\hline 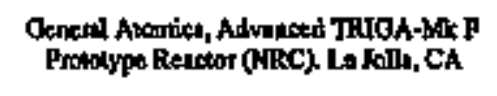 & $1,500,0$ & & 6000 & $\begin{array}{l}\text { TRITh-Mik F, } \\
\text { U-Zrhydridte. }\end{array}$ & Possossion onfy liconiso, 3/22/s5. \\
\hline 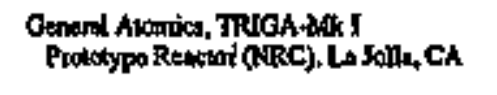 & 250.0 & & 5800 & $\begin{array}{l}\text { TRIGA-Mk I. } \\
\text { U-2R hydide } \\
\text { Owror }\end{array}$ & . \\
\hline 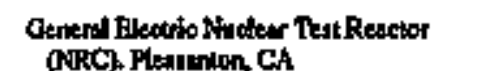 & 500.0 & . & 5700 & 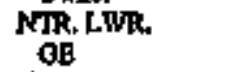 & \\
\hline $\begin{array}{l}\text { Neutioun Redilograpty Fusllity (DOE) } \\
\text { DTED. ID }\end{array}$ & 250,0 & & $\pi \mathbb{0}$ & $\begin{array}{l}\text { NRAD. } \\
\text { POaLIRIOA } \\
\text { DON, ANE. }\end{array}$ & \\
\hline 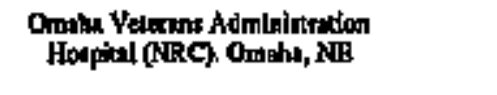 & 180 & & 5900 & $\begin{array}{l}\text { TRIGA-Mikt } \\
\text { J-Zh hydrido, } \\
\text { Gh }\end{array}$ & $\bar{n}$ \\
\hline 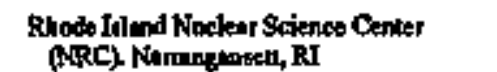 & $2,000.0$ & & 6400 & $\begin{array}{l}\text { RINGC. } \\
\text { Poot. RI }\end{array}$ & $' ?$ \\
\hline
\end{tabular}




\section{TEST, RESEARCH, AND UNIVERSTY REACTORS}

\section{PART I CIVILIAN REACTORS (DOMESTIC)}

D. General Research (Continued)

\begin{tabular}{|c|c|c|c|c|c|c|c|}
\hline $\begin{array}{l}\text { Name (Roguladory ogency). } \\
\text { Locallon }\end{array}$ & $\underset{\substack{\text { Ponper } \\
\text { pewoed }}}{\text { kw(t) }}$ & 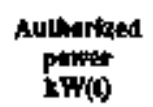 & $\begin{array}{l}\text { Powar } \\
\text { Mo espataly } \\
\text { out MW(e) }\end{array}$ & $\begin{array}{l}\text { Pomer } \\
\text { Deanow } \\
\text { MrW(T) }\end{array}$ & 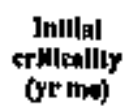 & 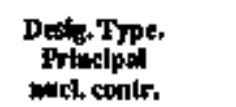 & Copmanent \\
\hline 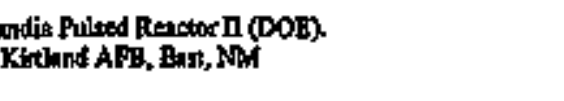 & & 25.0 & & & 6700 & $\begin{array}{l}\text { SFR-II. Bute } \\
\text { meul fast } \\
\text { bent. Sanding }\end{array}$ & Pulse, ulosdy atue. \\
\hline 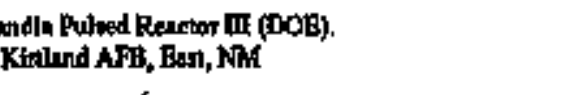 & & 23.0 & & & 7500 & SPR-II. Bars & Puloce, nuscidy ninte. \\
\hline 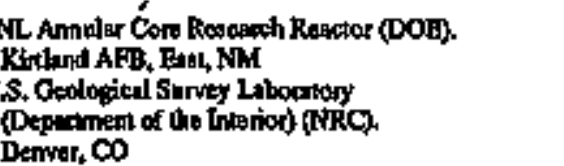 & & $\begin{array}{l}2,000.0 \\
1,000.0\end{array}$ & & & $\begin{array}{l}7800 \\
6900\end{array}$ & 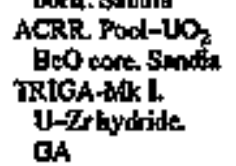 & 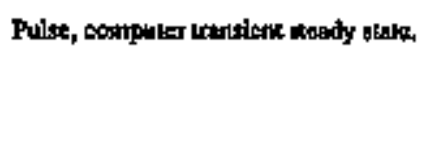 \\
\hline
\end{tabular}

\section{E. Univers/ty Research and Teachlng}

\section{OPRRALE}

- Arwon, Univemhy of (NRC)

atifomid, Irins, Jhwersity of ONRC

Ivine, CA

Comedt Univerity (NRC). It44a, MY

Comell Un'versiky Zevo Power Reackor

(NRC) laturs, NY

Fonida, Uhiversily of (NRC).

Grinesvilit, FL

Ceorgin methele of Technolosy (NRC).

Allania, OA

5,0000

datho Sinto Untrepity (NRC).

Pocetello, In

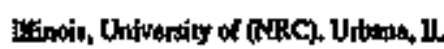

Dilinels, Untwersits of (NRC).

15000

Chanpaigan-U/tana, II.

5800 TRFGA-MkL.

68 of TRAGAMkL

uozr bydits$$
\text { GA }
$$

6200 miga-Hik I.

Uzzhrdide.

OA

6200 ZPR. Tink.

5900 UFTR.

$$
\text { UFTR. }
$$

Gnpting/water.

6400 GTRR

6700 Waler. CNNEC

Homeg. soliti

$A O N$

7100 LOPRA. U.Z

hydride. BA

600 TRtGA dik II.

U-Zx bydido.

ak

5900 UTR-10.

Onphiterwaler.

Onphiteh
As Inc.
Aublofized power in negfingiblo.

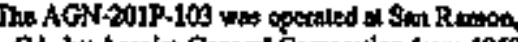

$C A$, by Aerojet-Genend Corportion frem 195

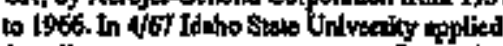

for a licensto is optrate the rasctor al Poodele.

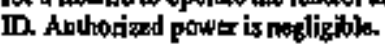


$--\quad$ U-Zrewerion

- Colloge (NRC).

Stew Yosk, NY

Muryind, Universily of (ARC)

2500

Mrenchnetlia, Uniwostily of (NRC)

10wel, MA

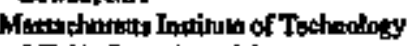

(NRC). Cunbridse, ALA

Mintign, Uniwanlyy of (Food Mtuclear

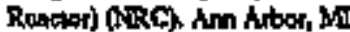

Masourl at Rolla, Uaiventiby of

ARCC, Rollh, HO

Milesouni, thiversily of (MRC)

Columbin, $M 0$

Now Hifterico, Univenily of (NRC).

Albe:querqua NA

6. North Crotina Siato Laiversily

(NRC). Rutigh, NC

Ohio Strte Uhtwerthy (NRC).

Cotombes, of

Oregen Stat Uaiventity (NRC).

Corvalli, OR

Pent Sule TRTOA Retutor (NRC).

Untivenuing Fak, PA

Poniwe Untrerrity (NRC)

West larryetle, IN

Reed Codlose (NRC). Pontingd, on

Soln Utwinerily of Now York

ONRC) Buttio, NY

$1,000,0$

$5,000.0$

$2,000.0$

2000

$10,000.0$

GA

6400 MCZPR. Thik.

Ald?

7400 THIGA.

Tank-'TroA

core GA

7400 ULR, Pool OE

5800 MTIR-II

Henvy walk

reflocked ACP

5700 PNR Pool. B\&W

61 00 UMR+R. POOL. CW

6600 MURR. Tulk:

Gworic

6600 AON-201M-t 12

Hompe. Solid.

ACW

Toxs Aald Universily (MRC),

Collegestubion, TX

Toxia AaM Uhinersily (RRC)

College Sintion, IX

Texal a Amatia, Untwersily of (RRC),

Anolia, TX
1,00630

500.0

1,00000

1,00000

1.8

250.0

2,0000

7200 PULSTAR POOL.

$$
\text { AMF }
$$

61 OSURR. Pool

lockloed

OSTR. U-2.

hyoride, ol

PSIR.

PooltTiOA

core $t A$

6200 PUt-1. Poot.

600 toskheed

totertoride,

$$
\text { th }
$$

6100 PULSTAR, Rool.

AMF

50 AON201M-106 Homeg, sclid. Aar

1,00000

6100 TRIGh U.2

8900

TRIGA-Mk II
U-Zs hystide.

aA
Aunhoriand power is neqfifible.

ACW-20LM-112 wat opentixd an the Undversty of Culifonia, Boikeloy, betinnting to J967. The

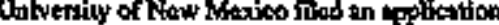
in 466 for triation and resonstruction of the rescter 4 in sice on is campus. The eactor nelitioved critically $\& x$ the Uniterdity of New Meticos on

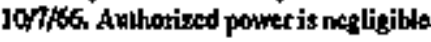

Owner Penniylvanla Stro Uhivotily. From 195\$ 10 1965,

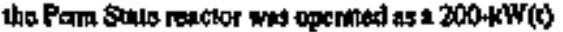
pod-lype macke fucled with bTR-1ypo elements.

The Siatc Univeraly of Now York at Entfílo rebclor

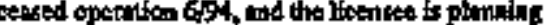
to decommission.

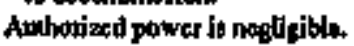

Tho Nerkar Säienco Canker Ronotor al Taxu A\&M Undversiky has been modilied for 1060-kW aleady.

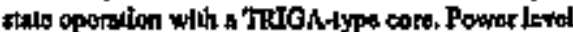
ber $100 \mathrm{XW}$ prior to modifticution in 1968 . 
E. Unversity Research and Teaching (Continued)

\begin{tabular}{|c|c|c|c|c|c|c|c|}
\hline Lat & $\begin{array}{c}\text { Power } \\
\text { copecily } \\
\text { mowity }\end{array}$ & 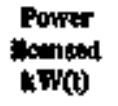 & $\begin{array}{l}\text { Aulhomked } \\
\text { power } \\
\text { kW(b) }\end{array}$ & 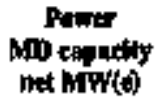 & $\begin{array}{l}\text { Power } \\
\text { Meshod } \\
\text { Mhit }\end{array}$ & 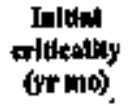 & 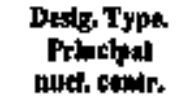 \\
\hline
\end{tabular}

Comment

Uab, Universily of (ARRC). Sult Lake

Cly, IT

Yisinith, Jutvertity of (NRCC).

Ohraloulsinitis, Vh

Washinglon sinto Uhivandy (NiRC).

Pulima, WA

100.0

7500 TRKOA-MK I.

U-Zrinyidide.

2,0000

1,00000

Whaontin, University of (RRC).

Worcenter Polylechnto inntiate (NRC).

Worcouter, Bar

6000 UVAR Pook.

600 OWDLABW

Pool-TRKar

core. GA

60 TRIGA.

Pool-tRrth

corr. GA

$5 \% 00$

No Desg. Poal.

QE
In 1967 the ofiginat MTR-ype core of the

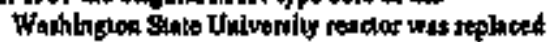
by a modified TRKA-type coro and antrol aynom, apd the Itendy-slate power tavel wal inoremes from 100 vo $1000 \mathrm{~kW}(0)$

The Univentity of Wleconsth reactor has been modited

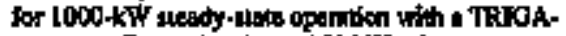
type para. Pow ilowal way $250 \mathrm{~kW}$ pior bo modififealion in $196 \%$.

\title{
1. MAATERIALS PFODUCTION
}

\section{PART II PRODUCTION REACTORS}

\author{
Nuns (OMntr) \\ OPEAHELB
}

Latoina

Noctery

deligner

Alken, SC

Do Ponk Heswy water

Do Pank Heswy water

ladiortat

pown Starlup

Coppluent

KReturtat (DOB)

5490

Cold anowdby.

\section{PROCESS DEVELOPMENT}

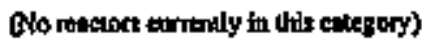




\section{DEFENSE POWER-REACTOA APPLICATIONS}

A. Remote Installations

QNo Teators andendly in this enkesory)

B. Propulston (Naval)

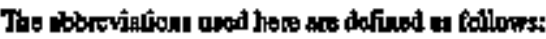

SSN, Subminino (tivelaar Propulision)

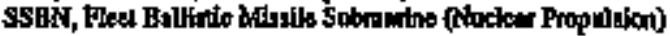

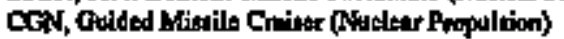

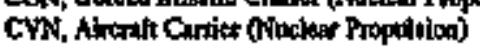

\section{Destganalon}

Shiptulles

Sturtup

\section{OPERADLE}

$\Xi \quad$ DANUAL WEBSTER

SAM RAYBUR

USSS KAMRRAMAREA

USS JAMES K, POLK

USS GRAYINKG

USs POGY

USS SUNFISF

USS SAND LAAFCB

USS EAWKBLL

USS SPADEPIS:

USSS FONEACX

USS NARWHAL

USS PNTADO

USS TRBPANC

USS Ethltest

USS ARCA ERFSE

USS WTLLAM H, BATES

JSS BA'TFIS月

USS TUNNY

USB PARCHB

USSCAVALLA

USS L. MBNDELL RWVER

USS LOS ANAELES

USS PFIIADELIPHLA

USS MELAPE

USS GROTON

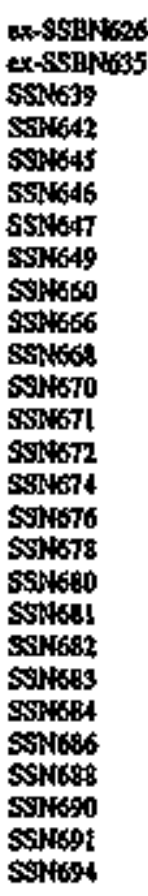

Erecaric Bon (Grolow)

Newport ilew

Ingells

680

Firearic Hoat (Grolow) 6600

kortanowilk $\quad 6900$

NYSCingells $\quad 7000$

GD (Quincy) $\quad 6800$

Portinomit 7100

Harelind 7000

Neupon Kew $\quad 690$

Newpon NEw: $\quad 6900$

Dieciric Bon (Gintom) 6900

Man isind $\quad 7000$

Glestric Bout (Gnoton) $\quad 7000$

Etectrite Host (Grolon) $\quad 7000$

Fileatric Hor (amolon)

infell $\quad 7200$

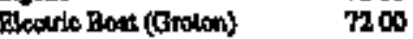

healli $\quad 7300$

ingella $\quad 7400$

Blectric Bast (Growot) $\quad 7200$

Newpon Now! 7400

Newpon New! 7600

Bhertrte Hoal (Omon) $\quad 7600$

Newport Nows 7700

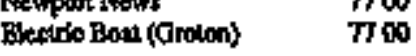

\section{PART III MILITARY REACTORS}

Cornitent

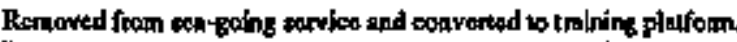

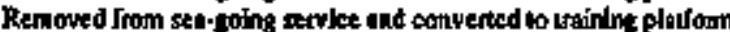




\section{DEFENSE POWER-AEACTOR APPLICATIONS}

\section{B. Propulsion (Navai) (Continuad)}

\begin{tabular}{|c|c|c|c|}
\hline Nane (nll owned by OS. Nary) & Deslgnelene & shpoolder & Bumtup \\
\hline USS BIRMINGHAM & SSNG95 & rlawpont Katud & 7800 \\
\hline USS DNDANAPOLS & S\$4 697 & Election Banl (Groton) & 7900 \\
\hline USS HRRHIRTON & SSWat: & Electric Bont (Groton) & 7900 \\
\hline USS JACKSONVILE & SSN 1698 & Blextric Banl (Grolon) & 7900 \\
\hline USS DAILAS & SSNTo0 & Batesice Bosl (Gmion) & 8000 \\
\hline USS LA XOLLA & Ssingol & Elcetnic Bonl (Groton) & 8100 \\
\hline USS PHOBNIX & SSN7on & Bafortrite Bats (Groton) & 8100 \\
\hline uss bostow & SSNFtos & Brectrde Bant (Groton) & 8100 \\
\hline UBS BALTMMORE & SSNTO4 & Electrio Boun (Groion) & 8200 \\
\hline USS CIIY OP CORPUS CERBSTI & SSNrios & Elecado Bonl (Groton) & 8200 \\
\hline USS AL BUQUERQUB & SSNDA6 & Eledrik Bonl (Omton) & 8200 \\
\hline DSS POAT Sincourt & ssw7ot & Elestrix Boul (Groton) & $\$ 300$ \\
\hline USSMONWBAPOLJS-SAINT PAUL. & ssin708 & Blecorylo Bont (Groton) & 500 \\
\hline USS HYMAN G, RICKOYER & SSTRO9 & Fecertio Boal (Groson) & 8400 \\
\hline USS AUGUSTA & $\operatorname{ses} 70$ & Blectido Bout (Groton) & 8400 \\
\hline USS SAN ERAKCISCO & SSIIII & Nowpont Nows & 8000 \\
\hline UNS ATLANTA & SSN7I2 & Nowpont Nowd & $\$ 100$ \\
\hline USS HOUSTON & ssivis & Newport Now: & 8200 \\
\hline USS NORFOLK & SSNV14 & Newpon Nows & 8300 \\
\hline USS BUFFALO & sSW715 & Newpont Nowd & 8300 \\
\hline USSSALT LAKB CITY & SSWFi6 & Nowrport Newa & 8300 \\
\hline USSOLYATH & SSNTI7 & Nowport Newa & 8400 \\
\hline USS HONOLULU & SSW7/8 & Newport Nowt & 8500 \\
\hline LSS PROVLERTCE & ssivis & Dletric Banl (Orolon) & 8500 \\
\hline Ws iIISgldeok & $\operatorname{ssin} 20$ & Blestric Banl (Grolon) & $\$ \$ \infty 00$ \\
\hline USS CHBCACO & Ssintzl & Nowpon Newi & $\$ 600$ \\
\hline USS KEY WEST & $\operatorname{ssin} 72$ & Netpont Nowt & 8700 \\
\hline USSORLAHOMA CITY & $\sin 723$ & Itwport Newt & 8700 \\
\hline USS LOUSVILIE & SSW724 & Hiectido Bont (Groton) & $\$ 600$ \\
\hline USS HELENA & $\operatorname{ssin} 25$ & Headso Benl (Oroton) & 8700 \\
\hline Uss OHIO & SSBAT26 & Elextrio Bout (Groton) & 5000 \\
\hline USS MKCHIOAN & SSENF2T & Eleotrio Bout (Gtaton) & 1200 \\
\hline USSFLORIDA & SSPN728 & Floctric Bow (Gotion) & $\div 00$ \\
\hline USS GEOROLA & 5SBNT29 & Electrio Bost (Gmion) & 0300 \\
\hline USS HENRY M. JACKSON & SSON730 & Electric Bow (Grotor) & 3400 \\
\hline USS ALABAMA & ssant3t & Eloctir Bow (Gototon) & $\pm+\infty$ \\
\hline USS ALASEA & $5 \sec / 732$ & Eleatrin Boek (Groten) & 8500 \\
\hline USS NBYADA & S5BN733 & Bleculde Bout (Geoton) & .600 \\
\hline USS TENWIESSER & SSBNTt3 & Blpetric Bonl (Grotan) & 8700 \\
\hline USS PEINSYLYANIA & SSEN735 & Electric Bosk (Gootion) & E\&OA \\
\hline USS WEST VERGNLA & ssant36 & Ekeclicic How (Conk:) & 9000 \\
\hline USS KENT & ssant3! & Eltchlis Boen (O, & 9000 \\
\hline UAS MARYLAND & SSEAT38 & Electrito Bont (Groton) & 9100 \\
\hline USS NEBRASKA & SSBA739 & Electrie Bont (Grolon) & 9300 \\
\hline
\end{tabular}




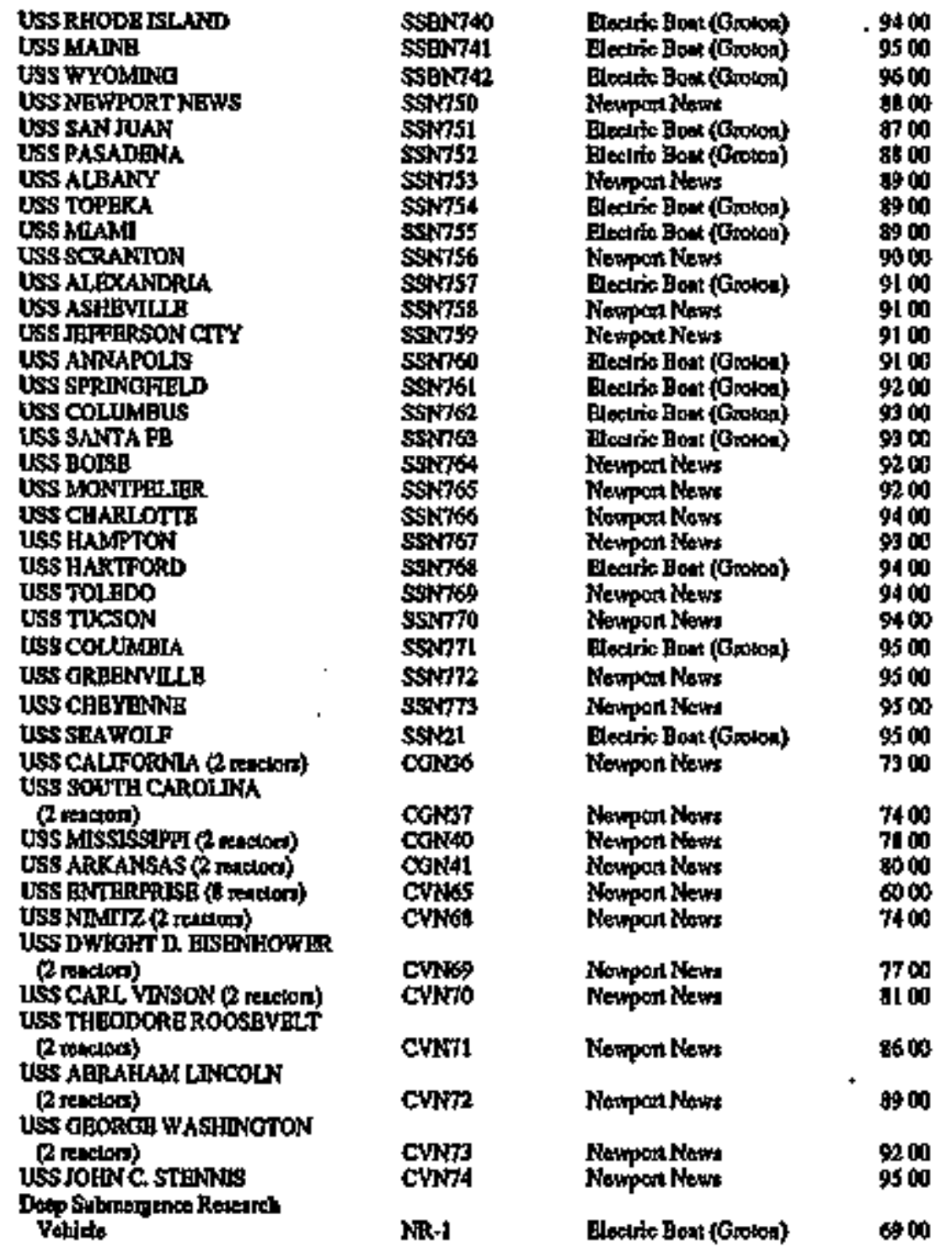




\section{DEFENSE POWERMEACTOR APPLICATIONS}

B. Propulsion (Waval) (Continued)

\begin{tabular}{|c|c|c|c|}
\hline Nune (all owmed by U.S. Navy) & Ilsesiguntion & Shlpballekr & Slartup \\
\hline \multicolumn{4}{|l|}{ DENNO BUIET } \\
\hline LOUISTANA & ssRint43 & Evedric Bon (Grolon) & \\
\hline CONNECTCUT & $\operatorname{sen} 02$ & Boctite Boen (Orolon) & \\
\hline HARRY S. TRLMAN & CVN75 & Newport New & \\
\hline RONALDREATAN & CVN76 & Newpant New1 & \\
\hline Nawe (all owned by U.S. Nory) & Designaltion & Shipbullatur & Slarlup \\
\hline $\begin{array}{l}\text { MANNND } \\
\text { (INRAMED) }\end{array}$ & $\operatorname{ssin} 23$ & Fectric Boar (Grolon) & \\
\hline
\end{tabular}

\section{DEVELOPHENTAL POWER}

\section{A. Electric-Power Experiments and Prototypes}

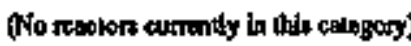

B. Propulsion Experiments and Prototypes
Nene (Omer), Lacutlog

\section{Optestate}

Modifiemions and Ad-iftion to Rescter

Fecllity (DOB), Wenl Mtibon, NY

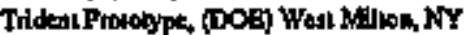

MLRP

SEO

\section{PART III MIILITARY REACTORS}

Iarlup

\begin{tabular}{|c|c|c|c|c|}
\hline Destifillon & $\begin{array}{c}\text { Power } \\
\text { copocdty } \\
\text { Eed } \\
\text { KF(t) }\end{array}$ & $\begin{array}{l}\text { Authorhed } \\
\text { power } \\
\text { in(t) }\end{array}$ & $\begin{array}{l}\text { Intatal } \\
\text { criticaliay } \\
\text { (er mo) }\end{array}$ & 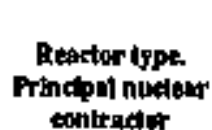 \\
\hline
\end{tabular}

Comment

\section{TEST AND RESEARCH}

A. Test

(No reacion cumedly th this cathory)
Praspluized water. Ladheed Murtin.

(Fonnery) (3).

Prtwinited walex. Loctheed Martin. (Fomety GE). 
B. Research

Namb (Ownar). Lactilun

\section{OPEARABL:}

Amred Fores i Rudiobtology Research tratiluke

DNA (DOD) Reyolined by NRC. Belhesda, MD

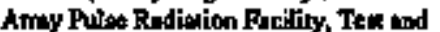

Evilunton Conmund (USK, Abesd:en, MD

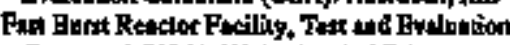

Commond (USA). White Sandr, NM

MeClellan Luclear Rodiation Center

(USAF, MrClellan AFB, $\mathrm{C}$

AFRRI

APRP

FBRF

MNRC

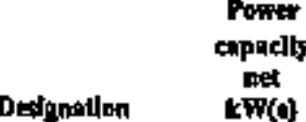

Aathorized Intels
pewer

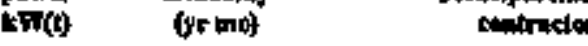

Reactor type.

Iticlpal neclear

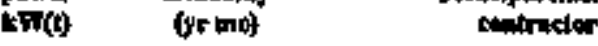

Comment

TRIOA-Mik F. GA

1,100

600

$10.0 \quad 6807 \quad \cdot \quad$ But, fapl, prosiph bara, UNC

$1006408 \quad$ Bart, Itst, poompt burst. Kanden

1,300:0 91 0l TRIOA Mod Mark IL GA

\section{PART N EXPORT REACTORS}

\section{POWER REACTORS}

A. Central-Station Eloctric Power Plants

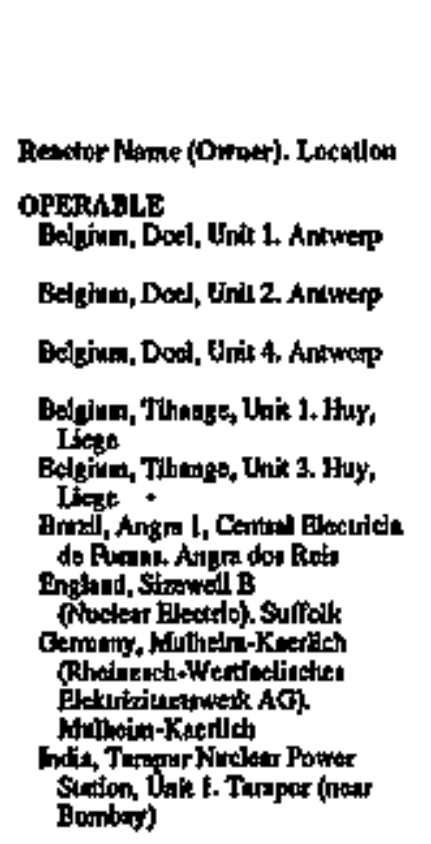

\begin{tabular}{|c|c|}
\hline $\begin{array}{c}\text { NRC } \\
\text { aport } \\
\text { lkewse } \\
\text { No+ and } \\
\text { date }\end{array}$ & 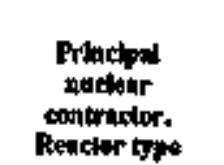 \\
\hline 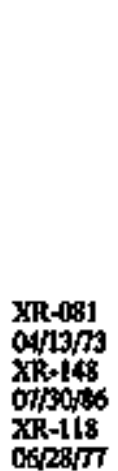 & 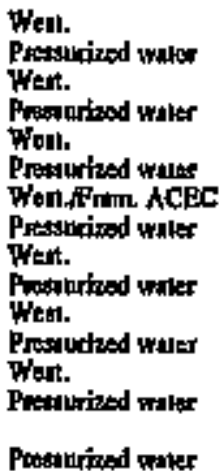 \\
\hline $\begin{array}{l}\text { xR-154 } \\
\text { ORfont64 }\end{array}$ & $\begin{array}{l}\text { GB. } \\
\text { Botling were }\end{array}$ \\
\hline
\end{tabular}

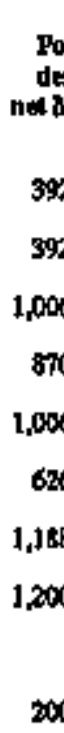

Powter

destm

Power

(0)

Author
bed
perer

Inliliak

anlt)

(yr mo)

Conmenet

$3920 \quad 1,19200$

7500

7500

6500

7500

5500

8500

940

n 10

6900
Fomer owner: Cempll Electidily Generaline Board. 


\section{POWER REACTORS}

\section{A. Centraf-Station Efectrle Power Plants (Continued)}

\begin{tabular}{|c|c|c|c|c|c|c|c|}
\hline Revelos Name (Onuer). Lacellew & 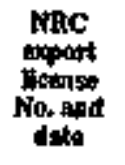 & $\begin{array}{l}\text { Primclyal } \\
\text { natichr } \\
\text { cointrictor. } \\
\text { feecter tope }\end{array}$ & $\begin{array}{c}\text { Poner } \\
\text { iesign } \\
\text { net } \operatorname{MW}(\mathbf{4})\end{array}$ & $\begin{array}{l}\text { Powar } \\
\text { MW(t) }\end{array}$ & $\begin{array}{l}\text { Aathor. } \\
\text { beal } \\
\text { pawe: } \\
\text { rwich }\end{array}$ & $\begin{array}{c}\text { Inlilal } \\
\text { crill- } \\
\text { callity } \\
(\mathbf{m}, \mathrm{no})\end{array}$ & Comanent \\
\hline 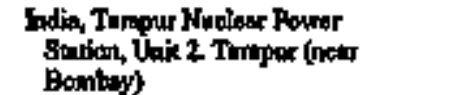 & $\begin{array}{l}\text { XR-0S4 } \\
\text { orpmist }\end{array}$ & $\begin{array}{l}\text { GR. } \\
\text { boiling writer }\end{array}$ & 200,0 & 707.0 & & 9900 & \\
\hline 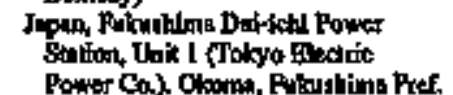 & $\begin{array}{l}\text { XR-006 } \\
\text { OAHLINT }\end{array}$ & $\begin{array}{l}\text { GR } \\
\text { Bolting onter }\end{array}$ & 4390 & 1,98000 & & 7010 & \\
\hline 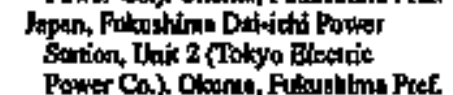 & $\begin{array}{l}\text { XR-072 } \\
04 / 227 \% 0\end{array}$ & $\begin{array}{l}\text { GE, Tosbib. } \\
\text { Bolling water }\end{array}$ & 7600 & 2,3810 & & 7305 & \\
\hline 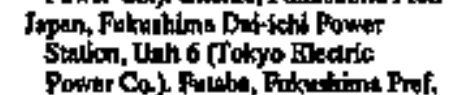 & $\begin{array}{l}\text { XR-0og4 } \\
05 / 5 / 79\end{array}$ & $\begin{array}{l}\text { GE, Toulibe. } \\
\text { Boiling thenter }\end{array}$ & $1,067,0$ & $3,293.0$ & & 7900 & \\
\hline 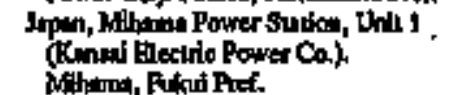 & $\begin{array}{l}\text { XR-0)T } \\
\text { OWLFTST }\end{array}$ & $\begin{array}{l}\text { Wen, Mollabilil. } \\
\text { Prnteminged waver }\end{array}$ & 320.0 & $1,091.0$ & & 3007 & \\
\hline 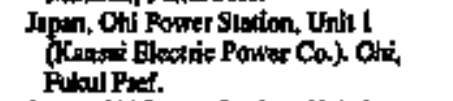 & $\begin{array}{l}\text { XR-018 } \\
\text { OWIT/73 }\end{array}$ & Wesl, Milinditahl. & 1,$120 ; 0$ & $3,420.0$ & & $n 12$ & \\
\hline 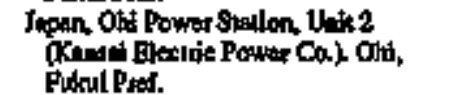 & $\begin{array}{l}\mathrm{XR}-0 \mathrm{~g} 2 \\
\mathrm{OH} / \mathrm{H} / \mathrm{T}\end{array}$ & 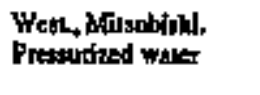 & 1,12000 & $3,423.0$ & & 700 & \\
\hline Japan, Tukghand Powner Sinlion, & $\begin{array}{l}\mathbf{x R}-019 \\
07 / 23 n 1\end{array}$ & $\begin{array}{l}\text { Wex, Misubidti. } \\
\text { Poestarled water }\end{array}$ & 780.0 & 2,4400 & & 7403 & \\
\hline 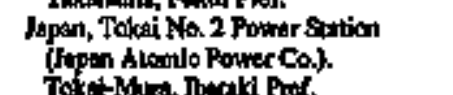 & $\begin{array}{l}\text { XR-0085 } \\
0 \leqslant / 25 / 73\end{array}$ & $\begin{array}{l}\text { OF, Hiachis Shinize. } \\
\text { Boting water }\end{array}$ & $1,0080,0$ & $3,293,0$ & & $7 \$ 01$ & \\
\hline 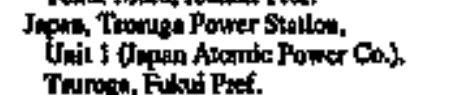 & 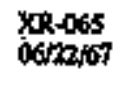 & $\begin{array}{l}\text { GE } \\
\text { Bolling wuer }\end{array}$ & 341.0 & $1,064: 0$ & & 69 is & \\
\hline 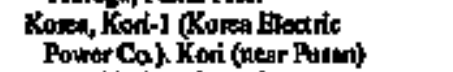 & $\begin{array}{l}\text { XRogy } \\
05,00 / 73\end{array}$ & West. & $\$ 64.0$ & \rfloor, 729.0 & & 7800 & Fonnonty, Koree, Unill 1. \\
\hline 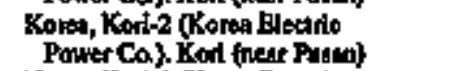 & $\begin{array}{l}\text { XR-119 } \\
0403 / 7\end{array}$ & $\begin{array}{l}\text { Went. } \\
\text { Proanuilized wher }\end{array}$ & 605.0 & $1,876.0$ & & 8300 & Pomworly, Korea, Unit 2. \\
\hline $\begin{array}{l}\text { Xoro, Kort } 3 \text { (Korea Blodric } \\
\text { Power Ca). Kod (a }\end{array}$ & $\begin{array}{l}X_{1}-131 \\
10 j 0478\end{array}$ & $\begin{array}{l}\text { Wen. } \\
\text { Presnurized water }\end{array}$ & 900.6 & $2, n 5,0$ & & 8500 & Formerly, Korra, Unit s. \\
\hline 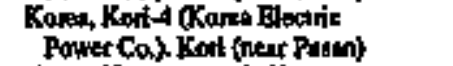 & $\begin{array}{l}\text { XR+13t } \\
\text { Joloans }\end{array}$ & $\begin{array}{l}\text { West. } \\
\text { Presporlsed wher }\end{array}$ & 900.0 & $2,775.0$ & & 6500 & Fomtenty, Kora, Unil 6. \\
\hline 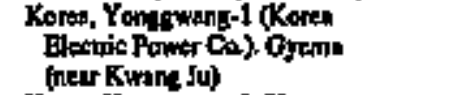 & $\begin{array}{l}\text { XR-133 } \\
\text { O9:24:30 }\end{array}$ & $\begin{array}{l}\text { Want. } \\
\text { Prestoriaed waver }\end{array}$ & 9000 & $2,775,0$ & & 8600 & Fonpenty, Korra, Unik ?. \\
\hline 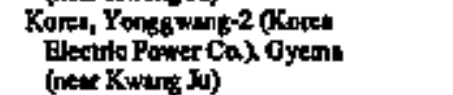 & $\begin{array}{l}\text { XR-133 } \\
0992-180\end{array}$ & $\begin{array}{l}\text { Weat. } \\
\text { Prestariand water }\end{array}$ & 9000 & $2, \pi 7.0$ & & 8700 & Pommenty, Koren, Unk 4. \\
\hline 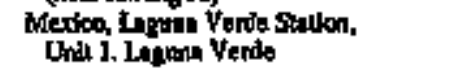 & $\underset{0 \mathrm{XR}-0 \% 5}{0 / 74}$ & 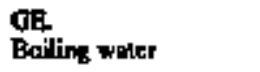 & 6540 & $1,931,010$ & & 部 [I & Contumtarill aperexion, 7 . \\
\hline
\end{tabular}

\section{PAFT IV EXPOFT REACTORS}




\begin{tabular}{|c|c|c|c|c|c|}
\hline 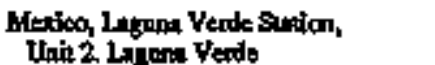 & XR-102 & GB. & GSAD & $1,931.0$ & 9400 \\
\hline $\begin{array}{l}\text { Nethetimdt, Dodewhind (GKO), } \\
\text { Dodenuad, Bemore }\end{array}$ & 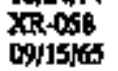 & $\begin{array}{l}\text { GB. } \\
\text { Bniling wutr }\end{array}$ & 55.0 & 183.0 & 6900 \\
\hline 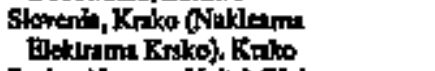 & $\begin{array}{l}x_{R}-107 \\
0 \leq 520 \pi 7\end{array}$ & 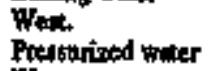 & 615.0 & 1,a52,0 & 2000 \\
\hline 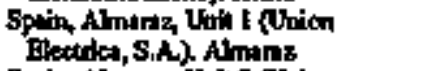 & 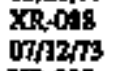 & $\begin{array}{l}\text { Wet. } \\
\text { Fredsurized ontor }\end{array}$ & 9020 & $2,696.0$ & 8100 \\
\hline 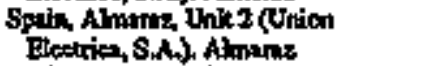 & $\begin{array}{l}\text { XRGBs } \\
\text { Dnt? }\end{array}$ & $\begin{array}{l}\text { Weat } \\
\text { Preapurixed whler }\end{array}$ & 9020 & $2,696,0$ & 6300 \\
\hline Spein, Awo, Unik I (FECSA), Areo & $\begin{array}{l}\text { XR-oso } \\
\text { (07/12/3 }\end{array}$ & $\begin{array}{l}\text { Wet } \\
\text { Presnurized water }\end{array}$ & 9020 & $3,696,0$ & 8300 \\
\hline Spoin, Avo, Untt 2 (FBCSA). Avo & $\begin{array}{l}\text { XR-095 } \\
0,02076\end{array}$ & $\begin{array}{l}\text { Wast. } \\
\text { Preupurized wouter }\end{array}$ & 90120 & 2,0060 & 8500 \\
\hline 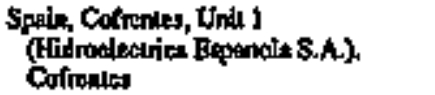 & 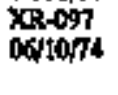 & $\begin{array}{l}\text { Gi } \\
\text { Boiling wakr }\end{array}$ & 975.0 & 2,90000 & 6400 \\
\hline 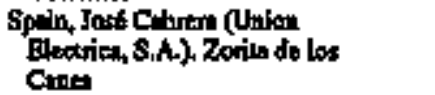 & $\begin{array}{l}\text { XR-059 } \\
\text { lor2er6s }\end{array}$ & $\begin{array}{l}\text { Weat. } \\
\text { Prejurized water }\end{array}$ & 160.0 & 510.0 & 6900 \\
\hline 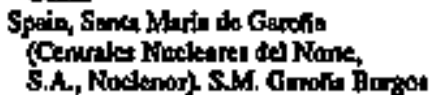 & XGR-064 & $\begin{array}{l}\text { GB. } \\
\text { Boiling wator }\end{array}$ & 40.0 & $1,381.0$ & 7000 \\
\hline 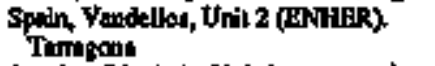 & $\begin{array}{l}\text { XR-122 } \\
09 / 13 / 87\end{array}$ & $\begin{array}{l}\text { Wert. } \\
\text { Prosingend woler }\end{array}$ & 92010 & $2,785,0$ & 6803 \\
\hline 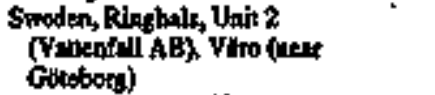 & $\begin{array}{l}\text { Xk1069 } \\
05,09 \% 69\end{array}$ & $\begin{array}{l}\text { Wett } \\
\text { Prosprized wuler }\end{array}$ & 875.0 & $2,600,0$ & 7410 \\
\hline 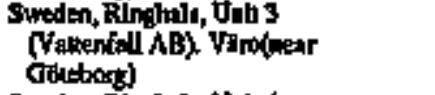 & $\begin{array}{l}\text { XR-09s } \\
\text { 02/02 }\end{array}$ & $\begin{array}{l}\text { West } \\
\text { Pretounized vilur }\end{array}$ & 9350 & $2,783.0$ & B] 00 \\
\hline 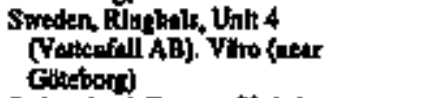 & $\begin{array}{l}\text { XR-103 } \\
\text { 10j21/75 }\end{array}$ & $\begin{array}{l}\text { Weat. } \\
\text { Prestud zed walor }\end{array}$ & 915.0 & $2,783.0$ & 1300 \\
\hline 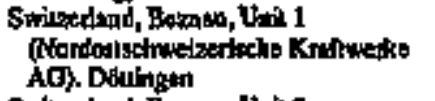 & 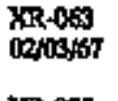 & 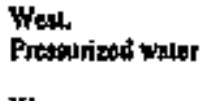 & 950.0 & $1,130.0$ & 6900 \\
\hline 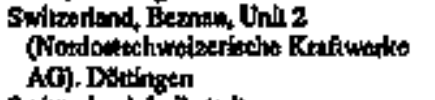 & $\begin{array}{l}\text { XR-070 } \\
11406+69\end{array}$ & $\begin{array}{l}\text { Wedl } \\
\text { Prespondzed wake }\end{array}$ & 350.6 & $5,130,0$ & 7100 \\
\hline 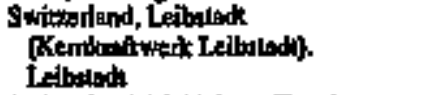 & $\begin{array}{l}\text { KR-50d } \\
\text { L2A175 }\end{array}$ & $\begin{array}{l}\text { dS. } \\
\text { Boiling waver }\end{array}$ & 3085.0 & $3,135.0$ & 8403 \\
\hline 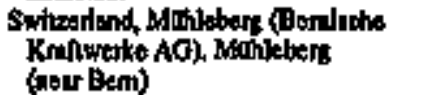 & $\begin{array}{l}\text { XR-06: } \\
\text { 10,04167 }\end{array}$ & GR & 320.0 & 9970 & 7200 \\
\hline 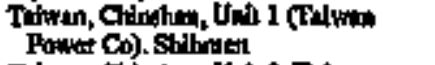 & $\begin{array}{l}\text { XR-10\%0 } \\
07 / 2172\end{array}$ & Be, & 604.0 & $1,7 n s, 0$ & $\pi 70$ \\
\hline 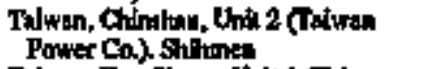 & 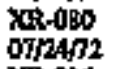 & CER & 604.0 & $1,775.0$ & 7411 \\
\hline 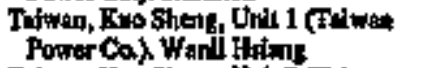 & $\begin{array}{l}\text { XR-096 } \\
041+7 / 4\end{array}$ & Ge. & 948.0 & $2,894,0$ & B1 02 \\
\hline 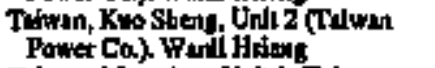 & $\begin{array}{l}\text { XRAS6 } \\
0417 \pi 4\end{array}$ & GF & 948,0 & $2,894,0$ & 0203 \\
\hline 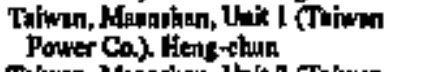 & 060 & $\begin{array}{l}\text { Wenl. } \\
\text { Pustoriced wator }\end{array}$ & 890.0 & $2,785.0$ & $B A 03$ \\
\hline 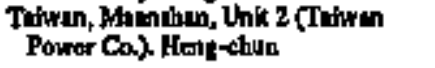 & $\begin{array}{l}\text { XR-113 } \\
\text { OARALA9 }\end{array}$ & $\begin{array}{l}\text { Weal. } \\
\text { Presinurized water }\end{array}$ & 890 & $2,785.0$ & BS 02 \\
\hline
\end{tabular}




\section{POWER REACTORS}

\section{A. Central-Station Elecirlc Power Plants (Continued)}

Reactor Nane (Omber), Latallon

\section{BEDNC BULLT}

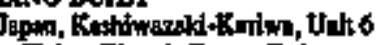
Tokyo Electic Power Co.)

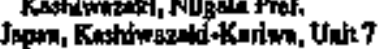

(Tokyo Blectilo Power Ca.).

(Takyo Hectilo Power Ca.

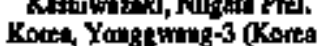

Bectric Power Col Gyomi (atar

Kwang Jo)

Kmpa, Yongavang d (Kores

Hlectric Powrer $C_{0}$ ). Oytme (pear

Kunos ful

Phili, ringes, Raptablic of the

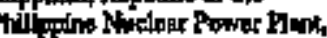

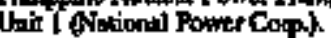

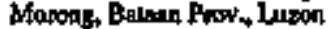

- Spais, Lamonta Unư ].

Lemion's

Spein, 1 mingit, Uht 2

Lemonis

Spein, Vatdecshaileros, Undt 1

(HE: Serillana de Blectrividend).

Volibestalloson, Budajor

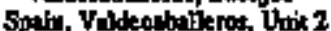

(GE; Soritina de Blectritidad).

Valdocabulloson, Buthajo:

\section{PLANINTI}

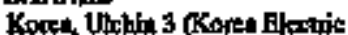

Powtr Ca). Kropumitusk-10

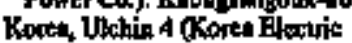

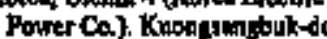

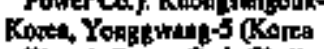

Electric Power Ca) Obollnum

Provinio

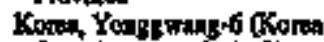

- Biesurte Power Co.), Chollmata

Provinoe

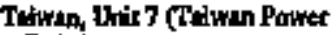

Co.th Langrocen

NAC

expont

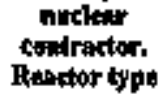

Prluclpat

CR, Toubibe, Huchl $\quad$,311.0 3,930.0

Adwreed boiling

or.

GL. Hitedis, Toahthe

XR-150

a4j6,67

Combor

Comb.

dact war

Odi16/8?

Xat -120

$05,06,80$

Personitixad waler

Warl.

Pressutined water

XR-089

$0 / 1273$

XR -0.99

$01 / 12 \pi$

$X R-110$

os, os

Weal.

Porsonize्d wala

Woul.

Pretibrized wales

Bolling water

XR-110 GE.

CSicosm

Boillne wher

$\mathbf{X R + 3 5 3}$

0400,5

XR.153

caras

XR-162

XR-162

Comb.

Protiunrined water

Comb.

Presulunined watar

Combi, Hisoing

Presonched waker

Comb., Fanjung
Preasubad wilor

02[17\%]

Thime Unls 9 (T)dwan Power

Co.). Inngment
Pawnr

begng rower

Mition

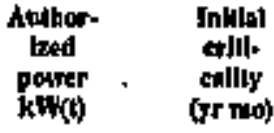

$1,311.0$

$3,930.0$

900.0

9000

6200

1.876 .0

\section{PART IV EXPORT REACTORS}

9500

960

bindef.

indaf.

Indef.

Inder.

Indet.

9500 26250

950.0 26250

1,0000

1,00000

$1,000.0$

9900

$1,000.0$

\section{Crament}

As of 12/95, 94.9\% comploked. Corrparcial oportion in tohedotod for J2/6.

As of 12/95, 10.95 centrilatod.

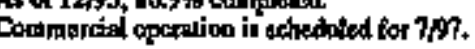

Alsp, Kone, tinil 11.

Also, Korea, Unid 12

\section{Listape penting}

Licinre perpting.

Tre MRC hat lasoed thae Hoenses 10 wendon for thit reteter: XR-13A, -135, tad -136. Oply the vendor who wini the contract with

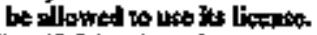

The NRC hes thuned uhese licenses ro wandon for hit ntectot: XR-134, -135, and -136 .

Only the veludor who wind the condrece will be allowed to use its lifernse 


\section{TEST, RESEARCH, AND TEACHING}

\section{c. Unlversity Research and Teaching (Contlmued)}

Rescler Nome (Omer). Locallon

Switamiant (uhiveriky of Baxel. Gessl

\section{CNILIAN}

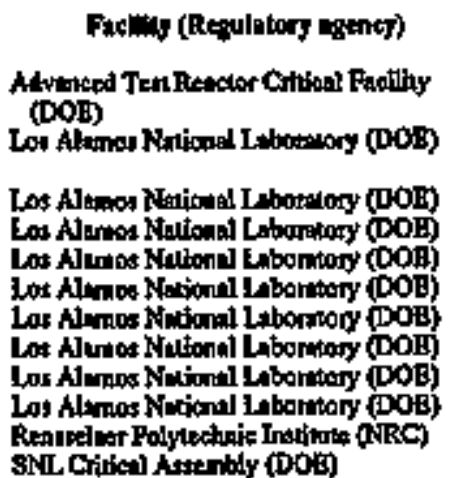

rat

SNL Critical Atrenbly (DOH)
Dratination

ATRC

Big Ton

Compar

Codivity

Hoonjocinb

Mins

SHFBA

SKUA

$\mathbf{x}$

\section{PARTIV EXPORT REACTORS}

$\mathrm{NaC}$

Priacipal

Whand

conlrattor

Resclar bype

desing Pamer

Aulher- loined

bod crill.

RW(y) (Grmo)

AON. 201-110

60 al

$2900 \quad 6500$

GA. TRYOA HE H

AR. UTR-10

GA TRKan-WR II

GA. TRKOA.MK II

AGN 201

AMTF. Pool (MTR)

sid

61 11

1000

6301

1000

6112

8200

2,0000

604

AON. 2]11+100

$\$ 800$
Comment

Nogthotble power. Shut down for menewd

Sil operaint licente.

Negligible patier.

Nopliziblo power.

The Nothediands reseanch reactor onl origindly opented at the Amalosdem Iritenitional

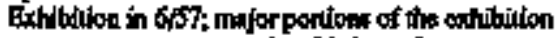

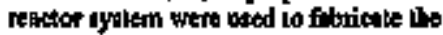
possent reactor.

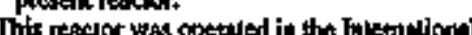

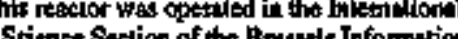

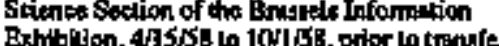

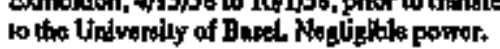

\section{PART V CRITICAL ASSEMELIES}

\begin{tabular}{|c|c|}
\hline $\mathrm{rat}_{\text {of }}$ & 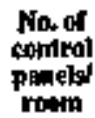 \\
\hline
\end{tabular}

DNAL Sive, II

Pajarito Sile, Los Nhanos, NM

Pajeinto Sito, Los Alumos, ND

Pejowios Stio, Los Alamos, NW

Pulutio Stre, Los Altmos, NDS

Painio Ster, Lot Alsmor, NM

Pajaito Sis, Las Almot, NM

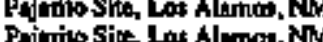

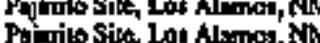

Prow, $1 Y$

Kinland AFB East, NM

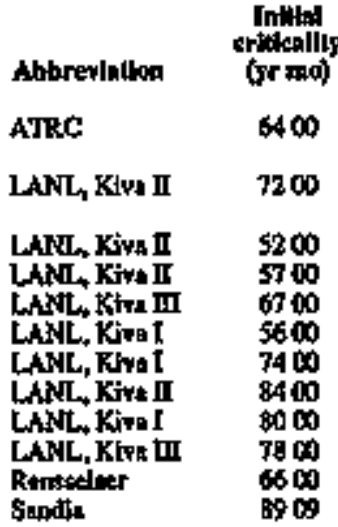

Coiment

ATR phyolict, corotooding and core-donign Housuremeati.

U(10)-raets cylladser in thidk melol ratlector.

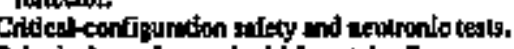

Sphericel meill coms in thick metel tellecios.

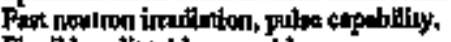

Flexible aplik 1ubles arsembly.

Ventical able absently maditino.

Vestical latike astentily.

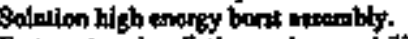

Fan nextron irrodtalion, polas appabitity.

Cilical expetiment sastmbly.

Spece poprer netulrondes; is thundown. 
2. MILITARY

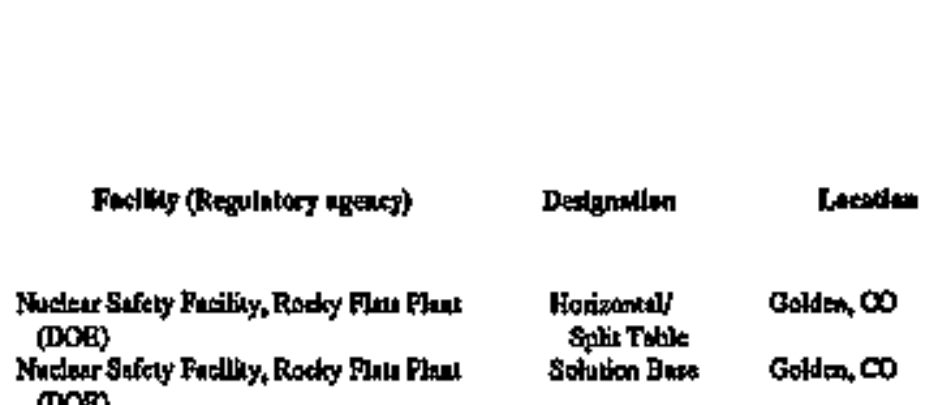

Mudear Sufety Fadillyy, Rocky Puna Phan (DOB)
PART $V$ CAITICAL ASSEMBLIES

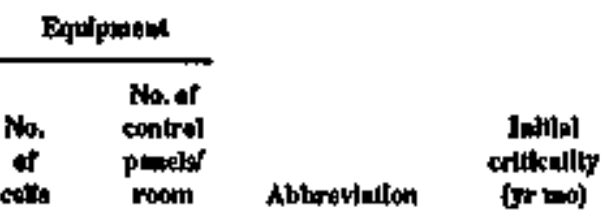

Comanent

\begin{tabular}{|c|c|c|}
\hline EPP-NSP & 6500 & 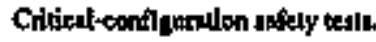 \\
\hline EP. & 6500 & Citlos teonffyration softet \\
\hline
\end{tabular}




\section{REACTORS AND FACILITIES SHUTDOWN OR DISMANTLED}




\section{REACTORS AND FACILITIES SHUTDOWN OR DISMANTLED}

\section{POWER REACTORS}

\section{A. Central-Station Electric Power Plants}

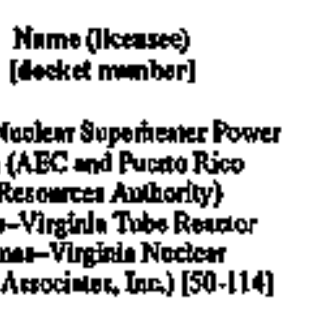

Droodon Nuckear Powtr Standon, Uatil 1 (Commonrealuh Edison Co.) 150-010)

Hik Rivar Reactos (AEC ind Rerol

Coopentive Power Aubodition)

Earicos Permi Alonio Poner Phat, Unit 1

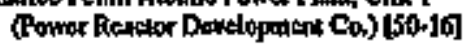

Fon \$1. Yrim Nucken Ceneming

Sintion (Rubitc Sesrike Compeny of

Colocesto) [50-26]

Hallan Muctar Power Fuality,

Sheldes Sulion (AEC) at Coniomers

Pablic Power Dabitits?

Humbolds bay Power Ptant, Unik 3

(Puelfie Ons \& Edectilo Co.] [50-133]

hodtan Polat Skalion, Unie I

(Connoltined Edion Co, of

Now Yol, Ino, $[50-3)$

1. Crope (Cletros) Nocker Geneming

Sintion (Dinlytand Power Coopernitve)

Puhtinder Aionio Plant ONortherd

Sorot Potwer Ca] [50-130]

Peuch Botlorn Atomice Powor Station, Unil I (Philadilphto Fled cic Co.) [50-171]

$\operatorname{mon}(\mathbf{1 0}$

\begin{tabular}{|c|c|c|c|c|c|c|c|}
\hline locallon & $\begin{array}{l}\text { Principal } \\
\text { nuctear } \\
\text { coniractor }\end{array}$ & Typt & $\begin{array}{l}\text { Power } \\
\text { Me capacily } \\
\text { net MW(O) }\end{array}$ & $\begin{array}{l}\text { Power } \\
\text { Wcensed } \\
\text { MW(t) }\end{array}$ & $\begin{array}{l}\text { Inltial } \\
\text { cillealiay } \\
\text { (yr mo) }\end{array}$ & 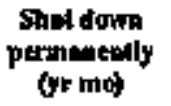 & Cormiatent \\
\hline Punka Hijoen, PR & Comb. & 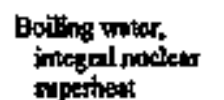 & 16.5 & 50.0 & 6400 & 5800 & \\
\hline Momit, IL & Wont. & 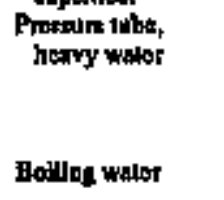 & 2000 & 700.0 & 5900 & 7. 10 & 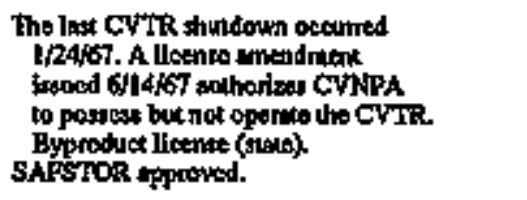 \\
\hline Elk Rives, MON & $A C$ & Boitlng walor & 220 & 58.2 & 6200 & 6.02 & 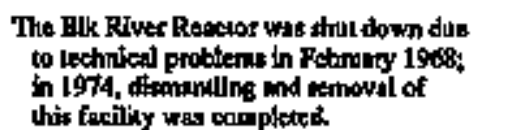 \\
\hline Lagoona Beaph, MI & PRDC & $\begin{array}{l}\text { Soditum cooled, } \\
\text { fasl }\end{array}$ & 60.9 & 200,0 & 600 & 7209 & 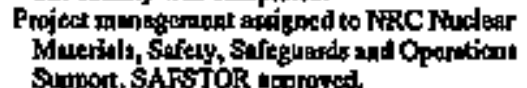 \\
\hline Pulorile, 00 & $\theta$ & $\begin{array}{l}\text { Higle tomperature, } \\
\text { gal cooled }\end{array}$ & 390,0 & 842.0 & 7401 & 8908 & DECOON nsproved. \\
\hline Euroka, CA & GE & Boillng water & 65.0 & 242.0 & $\infty 00$ & 3607 & 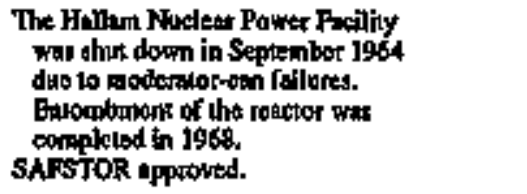 \\
\hline Bochanun, $N Y$ & Bew & $\begin{array}{l}\text { Presauflued } \\
\text { whist }\end{array}$ & 265.0 & 615.0 & 6200 & 7410 & 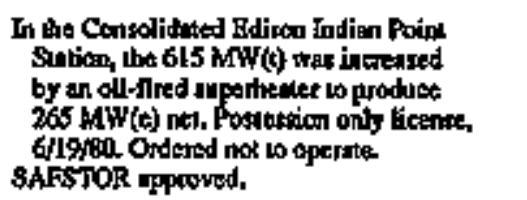 \\
\hline Sowx Fallh, SD & $A C$ & Boillat wals & 58.5 & 190.0 & 6400 & 6709 & 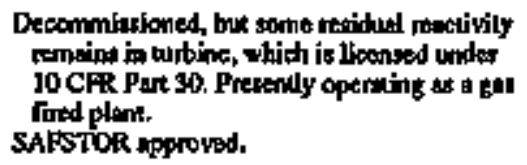 \\
\hline
\end{tabular}

\section{PART I CIVILIAN REACTORS (DOMESTIC)}

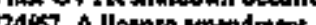

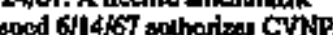

Dosian buc rot opento une CVTR

Byproduct licente (1tent).

The Hlk River Reecior wat thul itown dus

to Lechnteal probtens in Fstaning 19.6\% vis faciliky was canpleter.

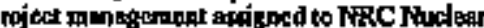
Muenhlo, Sofety, Safteglards and Opontion SUpport. SAFSTOR uprowed.

gat coolod SAESTOR MPPrOVA. 


\section{POWER REACTORS}

\section{A. Centrar-Station Electric Power Plants (Continued)}

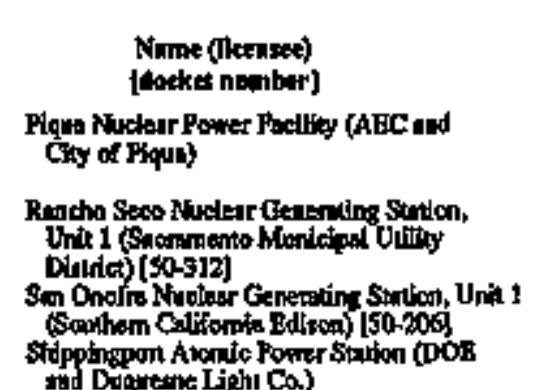

\begin{tabular}{|c|c|}
\hline 1acaldos & $\begin{array}{l}\text { Prhaclpad } \\
\text { ondloar } \\
\text { cowractor }\end{array}$ \\
\hline Ріянв, OH & Al \\
\hline
\end{tabular}

Clay Sucton, CA

Esw

Sen Chanealo, CA

Shlpphroport, PA

wert.

Wonl.

pothon At thd Digutente Lighi Co.

\section{PART I CIVILIAN REACTORS (DOMESTIC)}

Shormban Nurlear Paver Surticm

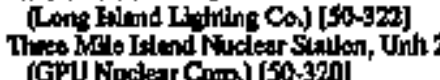

(GPU) Noclear Coop) [50-320]

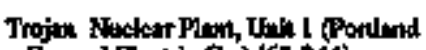

Generil Ractric Co, (SD-544)

Yapkeo Nuratear Powner Silailon (Yanken

Alonis Hectio Ca) [50-029]

\section{B. Dual-Purpose Plants}

Nome (Owner)

N Reteter (DOE)

\section{Brookhaven, $N Y$}

affodewom, PA

Presoon, OR

Rowre, MA

Locentron

Finfort Silin, WA

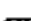

BAW

Heal.

weal.
Power MD capacts bet $M F(0)$

Type Orequitic cosied and notoderated

Proswutized

\section{Pretaunted}

Wates
13.4

873.

4960

6QD
Presinilzed
Fower

45.5

2,720

1,947

2300
Inlin

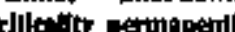

(yr me) (7r mo)

$6300 \quad 6600$

7409

1906

6705

5700
9211

6210
Commant

Iyg digamxienenk prognan for the Piqqun Kudeur Poner Fuotlity wiv.

compictod in. Pebruary 1969,
possegsion only licenss, 3192.

Possestion andy lizenue, 3999.

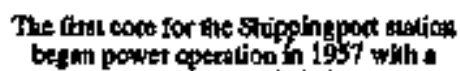
betent powet cpexdion in 1957 wih core becan power operation is ] With a caputily ming of 0 HW( $\mathrm{W}$. The thifu core, ilight witcer breeder reathor (LWBR), beqak power open-

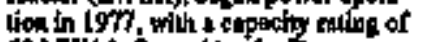

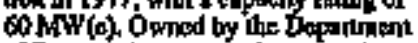
of Bnoridy, the mescior plant wats thent down on Oet. 1, 19:2. Defuelbit wis completed in Saptumbor 1994. Decommitestonting was comploted is Deceraber 1989, und the tile wat riclomed

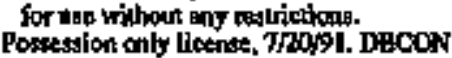
approwed,

\begin{tabular}{|c|c|c|c|}
\hline $2,436,0$ & 8502 & 9l of & 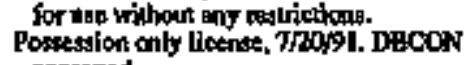 \\
\hline $2, m 2,0$ & 7800 & 7900 & 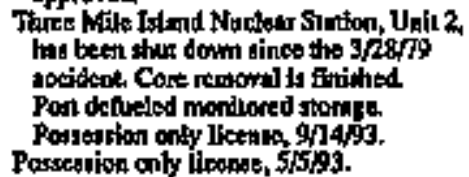 \\
\hline 6000 & 6004 & $9 / 02$ & 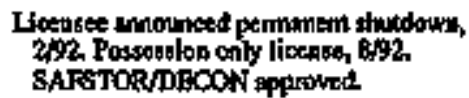 \\
\hline
\end{tabular}

Principal Power Power Inbal

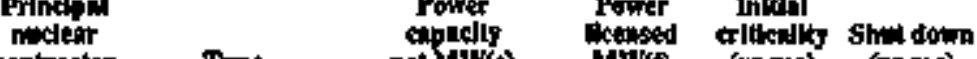

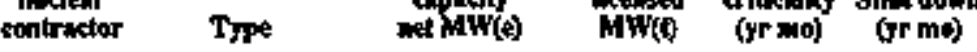

$8600 \quad 4000.0 \quad 612 \quad 9100$

9100
N Ration, \& DOE+otoned tescter for
WHC Craphilite

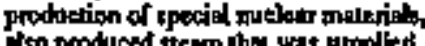
iso produced ttean the wat unplied

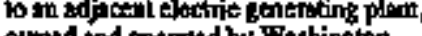
Pubile Power Supply Systens The reale Power Suppiyy stater to unation

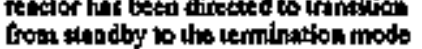
in preparation Jor decommissionipy. 


\section{Propulstion (Marttime)}

\begin{tabular}{|c|c|c|c|c|c|c|c|c|}
\hline $\begin{array}{l}\text { Nome modler owptr } \\
\text { [docket menber] }\end{array}$ & Npdenr desgner & Shlpouterter & Trpe & $\begin{array}{l}\text { Mrouinmm } \\
\text { shan } \\
\text { bordepone. }\end{array}$ & $\begin{array}{l}\text { Lumsed } \\
\text { power } \\
\text { MW(t) }\end{array}$ & $\begin{array}{c}\text { start. } \\
\text { up }\end{array}$ & 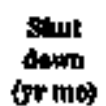 & Cosminent \\
\hline 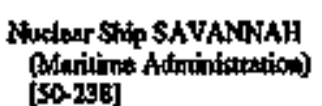 & BAs $\mathbf{W}$ & NYsc & $\begin{array}{l}\text { Protorized } \\
\text { wher }\end{array}$ & 22,000 & 800 & $610 b$ & 7100 & In SAFGTOR, potsession only licente. \\
\hline
\end{tabular}

\section{EXPERMENTAL POWER-REACTOR SYSTEMS}

\section{A. Electrite-Power Systems}

\begin{tabular}{|c|c|c|c|c|c|c|c|c|}
\hline 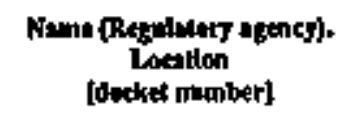 & 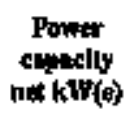 & $\begin{array}{l}\text { Powner } \\
\text { toenged } \\
\text { xhip }\end{array}$ & $\begin{array}{l}\text { Ambinorlzed } \\
\text { pporer } \\
\text { kW(t) }\end{array}$ & 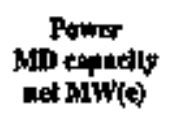 & $\begin{array}{l}\text { Purnat } \\
\text { Ilcenstid } \\
\text { Mibrit) }\end{array}$ & $\begin{array}{c}\text { Inlual } \\
\text { ctitlectly } \\
\text { (rr Jno) }\end{array}$ & $\begin{array}{c}\text { shut } \\
\text { dowim } \\
\text { (jrme) }\end{array}$ & $\begin{array}{l}\text { Deskg. Thpe } \\
\text { Printipul } \\
\text { mel. contr. }\end{array}$ \\
\hline $\begin{array}{l}\text { ollng Reacior Brpotimeal } \\
\text { No. I (DOE). NEIL Sin, ID }\end{array}$ & & $1,400.0$ & & & & 5300 & $\$ 400$ & $\begin{array}{l}\text { Borkx-1 } \\
\text { Boliting } \\
\text { waver ANL }\end{array}$ \\
\hline 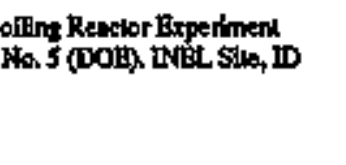 & $2,600,0$ & 20,0000 & & & & 6200 & 6400 & 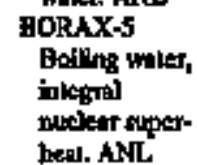 \\
\hline 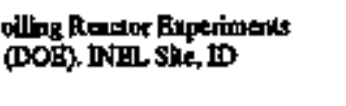 & $2,400: 0$ & $15,500.0$ & & & & 5400 & 5806 & $\begin{array}{l}\text { BORAX-2,-3) } \\
\text { Bolltikg wal } \\
\text { ANL }\end{array}$ \\
\hline
\end{tabular}

\section{ESADA Vullecino Kaperimewol} Siptitem Reuctor (KIRC) Plomanulon, $\mathrm{CA}[50-1 \% 30]$

\section{Experinand Boryllinen Oxids}

\section{Expainent Poiling Waver}

$4,000.0100,0000$

17.0000

$10,000.0$

Reacter (DOD). Argane, IL

\begin{tabular}{|c|c|c|}
\hline 6300 & 5702 & 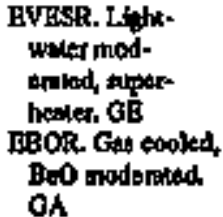 \\
\hline 500 & 5706 & $\begin{array}{c}\text { EBWR. Hoillne } \\
\text { wwer. AML }\end{array}$ \\
\hline
\end{tabular}

This fodlity was ordegindly bult tad opetated in 1954 an the Boling Rescor No. 2 (BORAX 2). What tho wddtiton of a witogemorter, it opented darting 1955 4 GORAX-3 and on 7/hiss producod miftiotent efectricluy lo Ilght wid power Artio, II- U.S, fiat BOR AX-4, \& futher modiffention, operalod from 12/56 to 6/55, when the axperiment wal shut dows.

Ownert Empire Simes Alomlo Developiase

Astodaler and Genoril Bedric Compuny. SAFSTOR, postesion orly liketile.

The BHOR retetor expertanenk wa tefminaled in Dectentor 5966 tuioe to eampletion of$$
\text { onsirution. }
$$

The EBWR achieved $100,000 \mathrm{~kW}(0) \mathrm{t} 1 / 11 / 52$. Oporsition of BBWR in the Boilling Wuter Program was closed ont 12/62. The reseter was used in suppon of the PIfloniun Recycte Progran and allited criliceslicy oting plocosium w Is pilncipal fuel 9/22/6S. In support of that program. it operated as potwor levols at hith as $70,000 \mathrm{~kW}$ (to Operation th thes program was eanpleted 6167. 


\section{EXPERIMENTAL POWER-REACTOR SYSTEMS}

\section{A. Electrlc-Power Systems (Contlnued)}

Name (Regulatory agency). Loctilt:

fankike nutiper

Experimentol Bexter ketacior

Ao, 1 (DOB). DNRL Sila, 10

\section{comactis}

Powar

net KW(s)

Power

Power

Anthorloes

130

$1,4060.0$

RW(0)

Power

at MWest

Powot

MWi()

millat

(Trinos

510

6400

Experinepitel Brroder Ro:artor II

20,0000

62,5000

(DOB), INBL Sice, II

61099409

$21,900,0$

\section{Bxperimencil Onunte Coolsd \\ Howy Wavor Comparends Test}

Resctor (DOB) SRL, Alten, SC

Homogeneous Rewans Frpetionen

No. I (DOE) Onk Ridse, TN

Honogeneciur Reactor Expesinato

Wh 2 (DOB) OAk Ridig, TN

tos Alanos Molien Butonitom

Rerctor Baperinesol (DOE).

Los Ahmol, Nht

Los Allarana Porwer Roactor

Exporinent No. 1 (DOD)

Los Alamoses No

\section{PART I CIVILIAN REACTORS (DOMESTIC)}

5,2000

Degig. Type.

Prindpil

nieci. contr.

tal. ANL

SaR-II. Sotium

conled, Bas. ANL

DOCR, Ga cooted, griphile mod.

artiod KE-AC

BOCR, Orgarite

cooled nind nod-

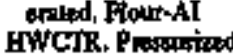

$61,10,00$

62006400

envy weler.

Do tont

HRE-L, Aqweout

bombeneous:

cohution $\left(\mathrm{JU}_{2} \mathrm{SO}_{4}\right)$

ORND

51006100 HाRE-2 Aquonu.

bomogeneoly

tolution (U) SO

ORNL

$1,000.0$

$6100 \quad 6900$

LAMFRE-1. Fas

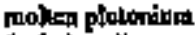

frolod sodiun

coolsd, CANL

howetereos

(photghotio aild)
Cerument

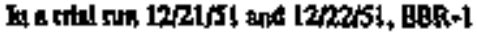

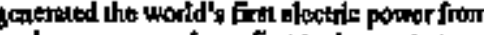
muckear enorgy apdi was first lo demongl mte, in 7/53, the feaslitility of bresing und UTe

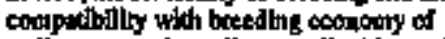

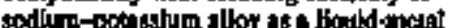
coolne por

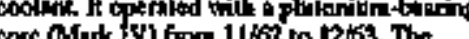
carc busk The for certy in 1564. Bho lanoto lat who 0 mo

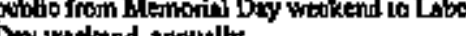
Dy

thous of

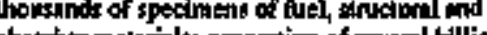

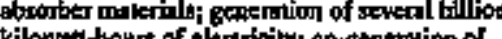
hom 1 the of

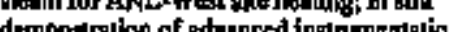

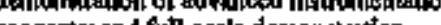

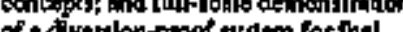
of a d venton- Esoof bystem for thel

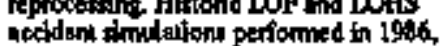

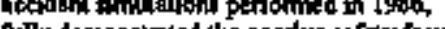

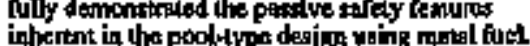
The BCCR proj test wad iaminalod 1/66 prior to becompletion of constrution.

BOCR cossimction was kemineted 12/62. The

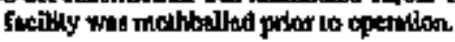




\section{Los Alimal Poure Reador \\ Exparinimt tho. 2 (10OB)}

Molesp Sall Reator Exporinent

(DOD), Onk RJdjo, tN

Onganic Modezented Rearto

Experiuent (DOE), INRL Site, II

Pusonitun Recratio Tes

Resctot (DOS) R3ehlind, WA

Surcom Nutkar Bxporiatanial

Rowetor Projo: (NRC)

Swont, PA [50. ]46]

DOF2 Sents Soment $\mathrm{CA}$

$3,0000 \quad 23,5000$

Southwer Expesintent il Futh Oxide

Rextor (SRC) Sxicklet, A

[00201]

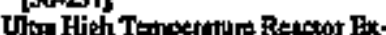
pariment (Dob), Lon Alam On, Non

Gisctos Hoiling Wotar Roactor

4
$5,70000 \quad 20,000,0$

1,0000

s,000

12 , 10015

No, c000

20,0000

20,0000

9,0000

$5,00000 \quad 33,000,0$
5900

5900

$6500 \quad 6900$

51006300

6000600

62001200

$500 \quad 6402$

69001200

com 700

5700 612
LAPRER Aqueou

hambitgeneodi

(phospharic waith.

MSRR. Single moden, groplikis modemied. ORNL OMRR. Ongmin

cooled and

modernivd. AI

waba, Anwy wale

modringed und

cooled. WHX

No Dess.

Presturized

wake. West

staptivio. $A$

SEFOR, Soting

sookod, fast. Ot

CHTREx, Holthw

cooled. LANL

VBWR. Boiltia
watar, $\mathrm{OB}$

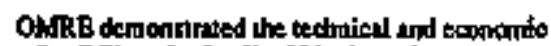

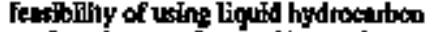
arphenyls as cooint ind fos modecticer.

Omart Suxion Theieur Bxperimendel Corp. In SAFSTOR, positestate oily lietase.

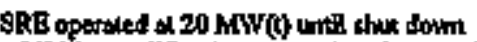

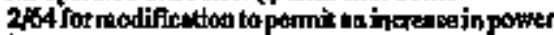
kvel $1030 \mathrm{MW}(0)$ On 12/2/66, detulivetion wos annowned. Owned: DOB and Sowlhem Cellifounls Bdison co.

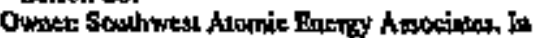

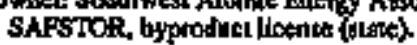

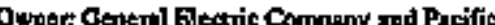
Ool \& Electic Ca. SAFSTOR eppromed

\section{Space Nuglear Auxdllary Power (SNAP)}

\section{Nimat (Hezultodory agencos). [docket nowber]} Rowher
copackly
ned klit(e)

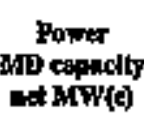

0.5

a.s

\begin{tabular}{|c|c|c|}
\hline $\begin{array}{c}\text { Pawar } \\
\text { Ilespons } \\
\text { RWo }\end{array}$ & $\begin{array}{l}\text { Atwhtbarlzed } \\
\text { power } \\
\text { ktwo }\end{array}$ & $\begin{array}{c}\text { Power } \\
\text { MD capuctly } \\
\text { et MW(e) }\end{array}$ \\
\hline
\end{tabular}

Powe

$\operatorname{Mrn}(\hat{)})$

500

sk.0

10.0

(DOE) Strita Sultin, CA

Eapetineat (DOE). Okk Ridyo, Tt

SyAP-0B Dordoponatal Reartor

(DOE) Santin Suuma, CA

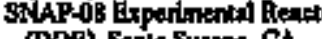

(ODB) Sand Sulan, CA

SXEAP-10A Flightix Syoten (DOB)

In odili, US

$\begin{array}{lll}600.0 & 6000 & 6900 \\ 600.0 & 6200 & 6500 \\ 39.0 & 6500 & 6500\end{array}$

\section{Desichation. \\ Type. Prindpal \\ nuckes}

contrindor

S2DS. Nok-cooted

AI

SER. NaK-sooled.

ANAP-TS:

Nak-cooled

AI-ORNI

SIDR. NaK-cooled. Al

segR Nakroodod

Alops-4.

NaK-cooled. AI
Comatent

Stors-4 openiod in onti Aprit-4ay 1965.

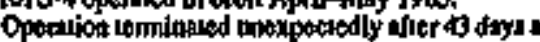

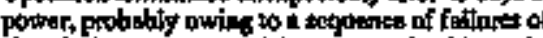
electrical capposends of the spatecrnlt with restl-

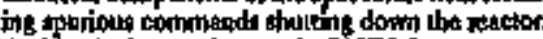
An id antical groond toat unil, stinFs-3,

opented thecestif ly for more then a yoar before being shut down in 1966. 


\section{EXPERWIENTAL POWER-REACTOR SYSTEMS}

B. Space Nuclear Auxillary Power (SNAP) (Continued)

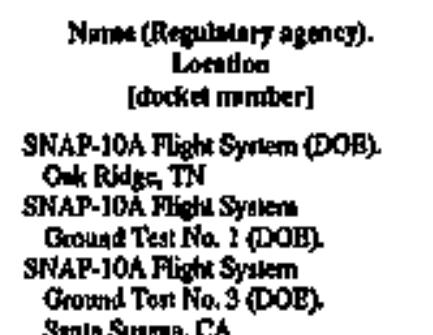

Pawer Paver Authostred Power

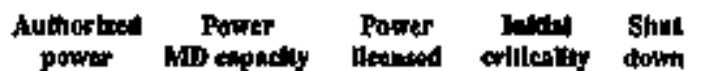

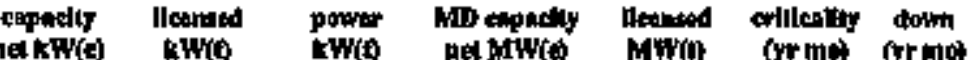

0.5 39.8 (Span)

0.5 350 6400 6400

0.5

390

$6400 \quad 6000$

anta Sumana $\mathrm{CA}$

\section{Space Propulsion}

$$
\begin{aligned}
& \text { Nond (Refolwory abtery). } \\
& \text { Locallon } \\
& \text { [dodkel number] }
\end{aligned}
$$

Puel Heweri Tax Bod (DOB). NRDS, NY

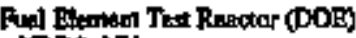 MN}

Fond Flamlex Ten Redutor (DOB) NoDs, NY

Gromd Buperimental Bngine

Buperimtin (DOH). NRDS, NV

Grotud Brpetimetixul Fingint

EMporinten (DOR). NRDS, NV

Nualear Rockea Buplos Reastor gaperimex (NIIRVA) (DOE) $\mathrm{NmDS}, \mathrm{NP}$

Nuelicts Rocken Enpins Reoctor Euprimenk (NRRVA) (COS) Nons, NV

Nuclout Rockal Englne Reaclor Bupriment (NER VA) (DOB) NRTS, Nt

Noctear Rocker Briging Rantor ExpentinenL (NERVA) (DOB)

\begin{tabular}{|c|c|c|c|c|c|c|c|}
\hline \multirow[t]{10}{*}{ 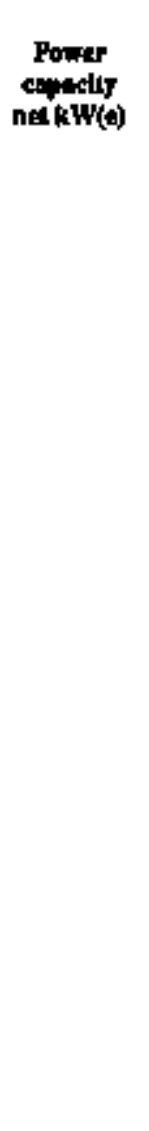 } & $\begin{array}{c}\text { Powar } \\
\text { llowied } \\
\text { RW(0) }\end{array}$ & $\begin{array}{l}\text { Authorbed } \\
\text { papter } \\
\text { Lwity }\end{array}$ & 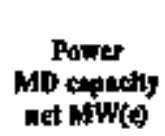 & 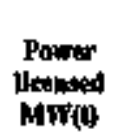 & 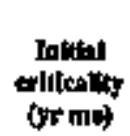 & 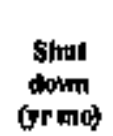 & 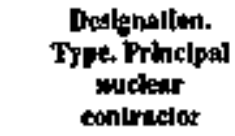 \\
\hline & & 14,00010 & & & 7200 & 7200 & 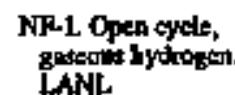 \\
\hline & & 5) 4,000:0 & & & lindef. & 6800 & $\begin{array}{l}\text { Perreg-1. Opea } \\
\text { cycle, liquitd } \\
\text { hydroner. LNNL }\end{array}$ \\
\hline & & S1A,0000 & & & Indef. & 7300 & $\begin{array}{l}\text { Pewres - Open } \\
\text { eyckts, Hoid }\end{array}$ \\
\hline & & 1,100,0600 & & & Indef. & 7300 & 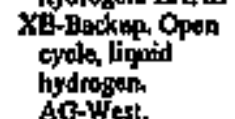 \\
\hline & & $1,100,0000$ & & & 6800 & 600 & $\begin{array}{l}\text { XE-Pine Open } \\
\text { cycto liguid } \\
\text { hydrogen. } \\
\text { AG-Well. }\end{array}$ \\
\hline & & 108,6020 & & & 6400 & 6400 & $\begin{array}{l}\text { NRX+A2 Opand } \\
\text { cycle topoli } \\
\text { hydrogen. } \\
\text { AG-WosL. }\end{array}$ \\
\hline & & $1,100,0000$ & & & 600 & 6500 & $\begin{array}{l}\text { NRX-ks, Open } \\
\text { cyde lopid } \\
\text { hydrogen. } \\
\text { AG+wool. }\end{array}$ \\
\hline & & $1,120,0000$ & & & 6600 & 6500 & $\begin{array}{l}\text { NRX-AS, Open } \\
\text { oycles lquid } \\
\text { hydrogen. } \\
\text { AG-Wos. }\end{array}$ \\
\hline & & $1,199,000,0$ & & & 6700 & 6700 & 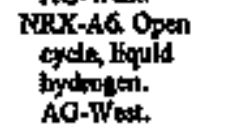 \\
\hline
\end{tabular}
NRDS, $\mathrm{NV}$

Comment

S10FS-4.

\section{S10ES-5.}

NaK-cooded. A

sioss-1.

Nak-cooled: AI

s10.3. 3 .

NaK-cooled. AI 
Nocke- Rockt Reatiox Engine Syum Ten (NERVA) (DOH. NROS, $\mathrm{NV}$

Tocker Rocket Ratedor Expetiliont (DOE), NRDS, NV

Nockes Rocket Rerctoe

Eroperiment (DOE) NRDS, NY

Nurdeur Rodvel Rewetor

Elyperiatienk (DOB), NRDS, NV

Nuctour Rodket Rewor

Expofitientx (DOE). NRDS, NV

Nineleur Rookel Rewcion

Nucleur Rocket Reacior

Experimem (COE). NROS, NV

Noctes RocketRenclor

Expeximenl (DOE), NRDS, NV

Nuctien Fodvel Rsabto

Frperipark (DOE), NRDS, NY

Nutrem Bodkel Regctor

Experimest (DOE). NRDS, NV

Neclear Rockest Ro:ator

Expenimeal (DOES, NADS, NV

Nucklar Rocket Roactor

Experimen (DOE) NRDS, NV
Eaperilaesx (DOG). NRDS, NV

$1,155,00000$

30,0000

85,00000

100,0000

300,0000

900,00000

sot,0000

$1,000,00020$

950,0000

$1,070,000,0$

$1,400,0000$

4,200,0000

\section{TEST, RESEARCH, AND UNIVERSITY REACTORS}

\section{A. General trradlation Test}

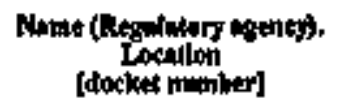

Enginexing tel Rewew (DOB) Net it

Feat Flux Tent Faditly (COB).

thantood Sile, wA

Gement Bitetric Tedind Reactor

(NRC). Ploapanion, Ch [50-70]

Poner
cepuchly
net kwite)

\section{Ponmer}

Aolhortzed
poprer
kW(t)

175, bonat

Power

Rower

$\operatorname{Mw}(0)$

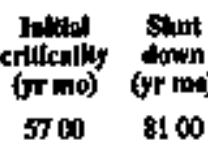

291,00010

sopocap
6600

$6 \infty$

NRX-A4EST.

Open cysles,

Hejietryorege

Aain

syete, gacoons

hydrogen. Lakit.

6000000 Kiwita Prine Open

$6000 \quad 6000 \quad$ Kiwita Print Op

$6000 \quad 6000 \quad$ Kiwiths, Opton

cyclo, gasoous

hydrogen. LANL

$6100 \quad 6100 \quad$ Kiwi-Bih. Open

creto, gaseoves

hysmgen LANL

$600 \quad 600$ KiwtBiB. Open

hydrogen LANL

$6200 \quad 6200 \quad \mathrm{Kiw}$-BdA, Open

ㄷy난, siondid

indeoten. IANL

$6400 \quad 6400$

i-94D. Op

epcis hiquid

bydoction. LANL

$64006400 \quad \mathrm{Kivi+B}$ (B. Open crdas Hequid

hydroges. LAIR

$65006500 \quad$ Phootas 1A. Open

cyele, Hiquild

hydoteter. LANT

Phoches 1B. Opes

cycto, liquild

hodrogen. LANE.

Propobas 2A. Opon

cyde, liquid

hydrogen LANL

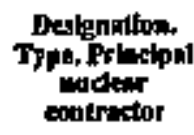

BTR, Tank KE+CR

$8002 \quad 9312$

$5800 \quad 7700$

\section{Cenmenes}

Rewation way uhat down in 1973 for motifices.

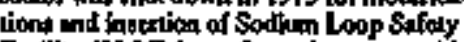

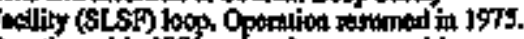

PFTP. Satum cooted The Pag Fin loop, WHC

GE'JR. Tank, OWrar, In SAPSTOR, pouassion only Lleense. 


\section{TEST, RESEARCH, AND UNIVERSTTY REACTOAS}

\section{A. General Irradtation Test (Contimued)}

\begin{tabular}{|c|c|c|c|c|c|c|c|}
\hline 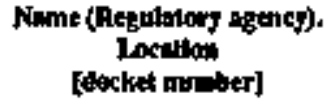 & $\begin{array}{c}\text { Power } \\
\text { espatiny } \\
\text { net kin(e) }\end{array}$ & $\begin{array}{c}\text { Power } \\
\text { liktimed } \\
\text { litit) }\end{array}$ & $\begin{array}{l}\text { Allhorked } \\
\text { powar } \\
\text { LW(s) }\end{array}$ & $\begin{array}{c}\text { Pawar } \\
\text { im topacthy } \\
\text { net } \operatorname{MrT}(e)\end{array}$ & 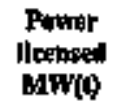 & $\begin{array}{c}\text { Inlilot } \\
\text { erikevligy } \\
\text { (gr mo) }\end{array}$ & $\underset{\text { Shim }}{\text { Shr }}$ \\
\hline 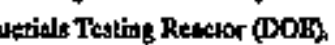 & & & $10,000.0$ & & & 5200 & 7000 \\
\hline
\end{tabular}

Pum Brook Rector Fecility ORRC)

Sintuaky, OH [50-30]

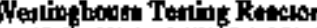

(anc); Watls Btiti, PA [5022]

60,0000

60,0000

\section{B. High-Power Researoh and Test}

Ampit Lentribry Resesrch Reectior$$
\text { (DOE), ADatis, IA }
$$

Bubcock \& Wilcos Nuolenr

Dovelooment Comer Teal Retelar

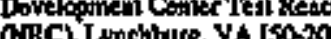

Chanco $\mathrm{PH}$ ito. 5 (DOE)

Argonis, it

Cintichen, Ino. Renctor (NRC)

Skes'lig Fostes, NY [50-5d]

Ine (NRC). Plaintoro, NJ [50-17]

Cak Bjidge Resaychl Rasclor

(DDF). Oak Ridga, TT

Outept Weal Roxtor (DOH), Lo

Aliman, NuH

Sindite Brointering Reorsos

(DOE), Kinland APG, Nb4

\section{Safeby Research and Test}

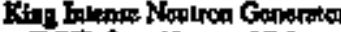

(DOE), Los Alamos, NM

K1w-Thantem Tea kearior

(DOE). NRDS, NV

Loan of Fwid Ten (DOF).

$$
\text { IN⿴囗十, ID }
$$

\section{PART I CIVILIAN HEACTORS (DOMESTIC)}

Destognallow.

Typor Frindipnl

muder

contractor

MIR. Tank

ORNLANL

Bjaw-Knox

$\$, 000,0$

6,0000

$5,000.0$

5,00000

$5,0000,0$

30,060.0

8,000,0

5,0000

$\begin{array}{lll}6100 & 700 & \text { NASA-TR. Tonk. } \\ 5900 & \text { MASA } \\ \text { WiR. THnk. } \\ \text { Owmer }\end{array}$

$6500 \quad 7700$

640070

\section{ALRR. Heany}

Whiter, AMP

BAWTH. Poa

5A 02 T9DS CP-5. Heovy.

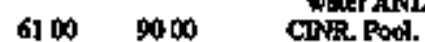

5007500 AMF

IRL. Pool.

AN

ORR. Tunk. ORNL

$5800 \quad 8700$

5600 9400

OWR. Trak

1ANI.

61007000 SER. Tanks Sindik

KGingtor.

Homoretreon

LANL

65006500 Khwi-TIR.

Riwianter Y A

LANT.

$\$ 5,000.0$

$7800 \quad 850$
OFT, Prominad water. HOAO-ID
In Augugt 1958 the MTR was opented with in

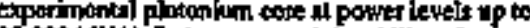
$30,000 \mathrm{~kW}(0)$. I denonalmbed the stilky of

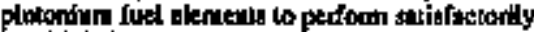
in a high-flux retentrok or ven retector. Operrition

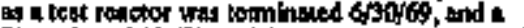
Plucotion-240 (flumenix) core was man In FY 1970 . Retetor was desomalabloned in 1974. In SAFSTOR, powposion Guly licessa.

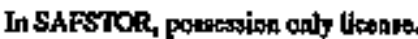

in SAFTTOR, byproduct Heenso (NRC).

DBCON appromed.

Lixenge teminated, $11 \mathrm{~A} / \mathrm{m}$

Shut down-Difueted.

Shut down-Dudnoled.

Transteol.

LOFT covered mont of the ooncerms related to Itathwaker sofuty. The Jirt leste were doone for NRC, and the lati eishin twere fono for a consorting of CECD couphtes and the U.S. The hast Iwo Issios-product releste tets messured reiteste and lrantiport of fission prodacts. There was an intentioned cose dim.

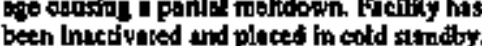


Power-Bom Fecilicy (DOF)

stap-10A Trenlen Teal to. 2

20,0000

(DOE), MEI, I

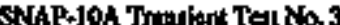

COEX, INEL, tD

Special Power Bxcuriton Reackor

Tra i 10,1 (DOE), Rafl, II)

Spedul Power theoriton Rexcker

To* N0. 2 (DOB), INEL, ID

Spocial PGwer Excortion Retctor

Ton No. 3 (DOE), DVEL, II

Spectal Powwer Bxcumlon Reaclor

Tou No, 4 (DOE), DEE, ID

D. General Research

Acetientor Pulted Fen Civicul

Astembly (NRC). Is Jolla, CA [50-253]

Amerietu Sind ard he. (NRC)

Mouluin Visw, CA

Anolor Cort Ruled Reation

(DOE), Btabind AFO, But, NOM

Argonec CP-3, noruth an

CP-3 (DOH) Pua Pute II

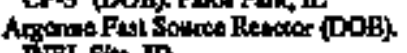

IN EL sith In

Agatue Low Power Reseiris

Rouctor (DOE), Argonur, II

\begin{tabular}{|c|c|c|}
\hline 6400 & 6400 & $\begin{array}{l}\text { SNAPTRANA, } \\
\text { H,O-tedkoted } \\
\text { SNAAP-10A } \\
\text { PPC-AI }\end{array}$ \\
\hline 5500 & 6400 & $\begin{array}{l}\text { SPERT-1. Ope } \\
\text { tunk. PFC }\end{array}$ \\
\hline 6000 & 6540 & $\begin{array}{l}\text { SPERT2. } \\
\text { Presturized } \\
\text { vatec. PRC }\end{array}$ \\
\hline 9840 & 6800 & 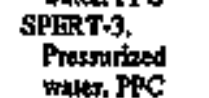 \\
\hline & 7000 & SPERI \\
\hline
\end{tabular}

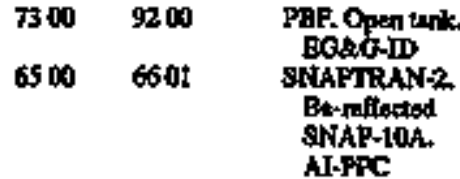

Argune Nationel Laboralary

(DOB), Artonne, IL

Aponos Toctar Aukenbly

Jor Untuerity Tnitrin

(DOA) Argonee, I.

Agsnis Theminl Sames

6700 7300 APFA-III Fan.

5800

UTR-t.

Oraphitite/tunter.
AS, Ine.

3000
$6700 \quad 7700$

ACFR. U-ZX hydride. OA

CP-3, HEWY viter. Mot 1 ab. AFSR. PMA ANL Jogemant Oraph

5900

62007000

$5700 \quad 7200$

$5700 \quad 7200$

$5700 \quad 8800$

ACH-201-108

Argowani (CP-1i).

Graphiteidwer.
Homog. 1olid. AON

ATSR. Taemal ANL
The SNAPIRAN wedes of experimems wet defigned to develop, in a I Inpt-bured

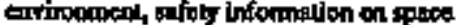
maxiliary power tesesen througl areurtion

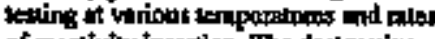
of restivity inserion. The dealnactive axporiments approach lit matimim credible

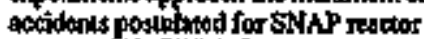

syalemn. SN $A$ PIRAN-1 was costeted in

STAPTRAW 2 jor dostroctive losting $t / 66$.

The APPA-III wat previousty opkeded as itio KUIRLA Prompt Critical Astembly ot

L wrence Livermore Nalional Iaboritory

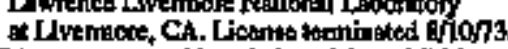

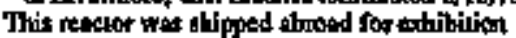

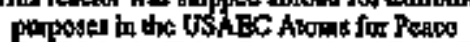

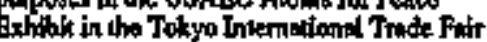
1959, apd in Caling, Elyph, and Latioro.

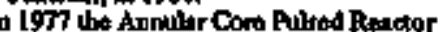
(ACPR) was shul down. ACher aplacernegl of fach

Aher the monbly and opestion of thlt rectior

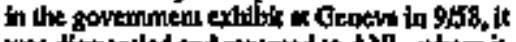

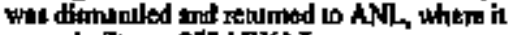

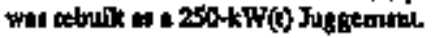

r




\section{TEST, RESEARCH, AND UNIVERSITY REACTORS}

\section{PART I CIVILIAN REACTORS (DOMESTIC)}

\section{General Fesearch (Contlnued)}

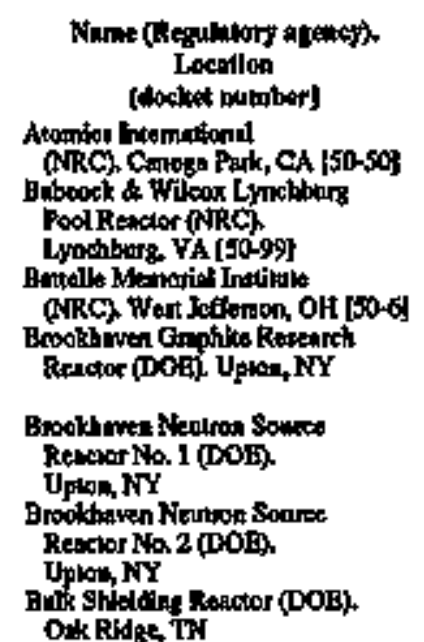

Rower

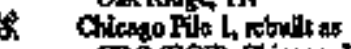

CP. (DOE), Gitago, II

Oertis-Wilght NuclearResesch

Intrarilory of the Commou-

Wealth of Pentyly win (NRC).

Quntanani, PA [S0-310]

DCA Demosumiton Reacior

(DOBh OAk Ritiso, TT

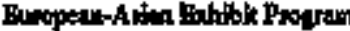

(DOE) OAl Ride, TT

\begin{tabular}{|c|c|c|c|c|c|c|c|}
\hline $\begin{array}{l}\text { Power } \\
\text { Hktesto } \\
\text { kW(t) }\end{array}$ & $\begin{array}{l}\text { Aulwortzed } \\
\text { power } \\
\text { kmon } \\
1,0000\end{array}$ & $\begin{array}{l}\text { Power } \\
\text { MD copacly } \\
\text { met } M W(\theta)\end{array}$ & 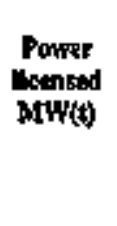 & 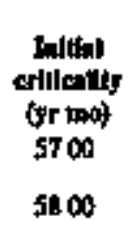 & 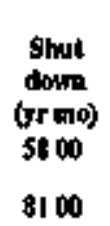 & 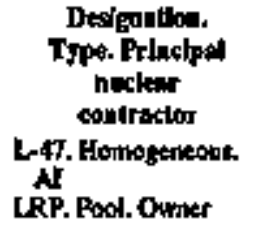 & 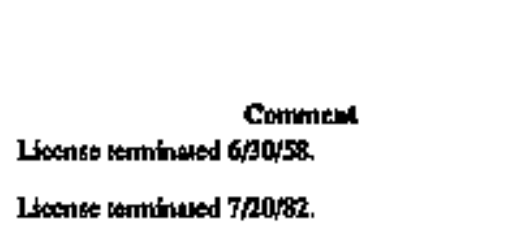 \\
\hline & 2,0000 & & & 5600 & 7400 & BRR, Pool, AMF & 1kcense terminaled $12 / 22 / 87$. \\
\hline & $20,000.0$ & & & 5000 & 6900 & $\begin{array}{l}\text { BGRR. Air cooted, } \\
\text { graphilo mod. }\end{array}$ & ${ }^{\circ}$ \\
\hline & 100.0 & & & 5800 & $70 \mathrm{da}$ & $\begin{array}{l}\text { SCREzo. Tuak AU, } \\
\text { kac }\end{array}$ & \\
\hline & 100,0 & & & 6500 & 7000 & $\begin{array}{l}\text { PHIRENIC, Task, AU, } \\
\text { Ine. }\end{array}$ & \\
\hline & $2,000,0$ & & & 5000 & 9100 & BSR. ROAl. ORRl & Sinn town-10 be defulted. \\
\hline & $1,000,0$ & & & $\$ 200$ & 5400 & $\begin{array}{l}\text { CP.2, Graphile } \\
\text { Met. Let. }\end{array}$ & 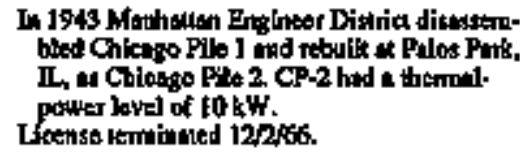 \\
\hline & 100 & & & 6900 & 6900 & $\begin{array}{l}\text { Demin Reac Pcol. } \\
\text { Lackleed }\end{array}$ & 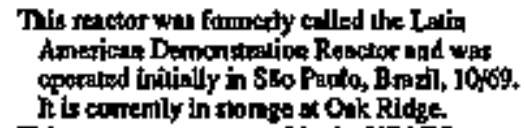 \\
\hline & 10.0 & & & 6300 & 6900 & $\begin{array}{l}\text { EASP. Puol. } \\
\text { Lockheed }\end{array}$ & 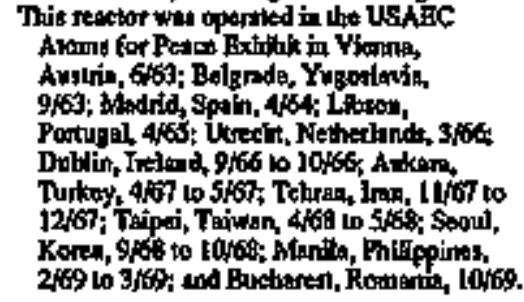 \\
\hline & & & & 670 & 7000 & $\begin{array}{l}\text { BNL/RS-1. FusL } \\
\text { BNL }\end{array}$ & \\
\hline & 300 & & & 60.00 & 5000 & $\begin{array}{l}\text { TRIGA-Bat II. Ut-Zr } \\
\text { hydilde. Owmer }\end{array}$ & 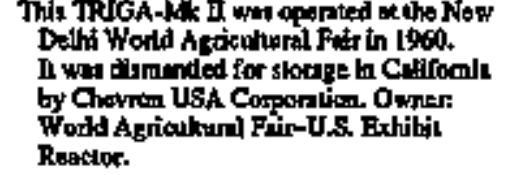 \\
\hline
\end{tabular}

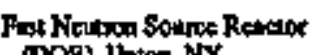
(DOB) U 1pton, NY

Genent Aloosine Tectuologites (DOB) St Dlope, CA 
Hian Fhydan Reanith Fieactor (DOE) Duk Rldye, TN

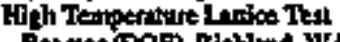

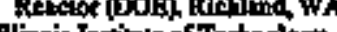

moil iantion of Tertancilosy

Reysarch lorthots (NRC)

Chirago, IL [50.]]

JANUS Reactor (DOD).

Antonite In,

Kineso Hoper'mest on Waler

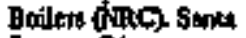

Snande Ch

Livetmose Pool Thpe Retatos

(DOE), Ltwermore, CA

Hvemone Wuer Boller

(DOE) 1 inemeth $C_{A}$

Locthoed Airnd Cop (ARC)

Drasomile, oA \{so-16t)

los Almos Fist Rinckor

DOts los Aluros, tos

Lor Almos LOpO Renctor

(DOB) L, As Alemos, NM

Los Almos Wuter Boliter

Los Alimes Winter Boiter

(DOE) Los Alamos, Nhs

L Lothtinn Stalo Ghiverity

thuoter Sckense Cender

(DOB), Bollom Rouge, 1A

Low Intengly' Tart Renciox

(DOE) OAk Ridge, TN

Imadimpen Faciliky (DOB)

Onk Ridge, TN

NASA Mtook-Up Renctor

Aitec) Stathitky, OH [50-185]

Neutron Radiogn phy Fańlits

(COB) tringond Sin, WA

Nocthrop Corpornte Labonicrie*

(NRC), HLmbome, CA [50-187]

Nucker Erferts Renctor (OOH).

NIS, NY
100.0

1,00000

3,00000

00
6200910

67007100

5600

6700

64009200

560060

5000000

53006100

60006000

$4600 \quad 3500$

$4460 \quad 4400$

44005000

50007460

$6500 \quad 6600$

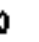

5000 6500 UTR Thenk.

1606 9100 CPNTL

69 7300 MUR. LWR.

Too 9000 Nodkeod

$6300 \quad 8600$

TRIOA-MK P. U.Zr

$6200 \quad 7000$
ORNL

hoditide. WHC hydrite. GA

modariled, PNL

ARR ( $-S A)$.

AI

Hortogemedot, AI

PTR. Tok.FW

B. Homogenesus.

Al Dogg, Pool.

Lockheed

pintonion foil,

merciny tooled.

19po

LANI

LANL

IANT.

SNARR Poral.

Sandin

(1) LLNLPPC
HFRR Fan bars
Shate dowa-Difuted

Ownert Ammoor Reseurch Fourdetion. 1 Sonte thinfund 4p87\%.

The KEWB cactos was opented bo AI from

1956 lo 1967 as the Kinjeto Expetenend etr

Waver Boikst. Owner: Rockwell Intendioank.

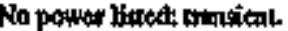

License teminuted 911050.

h 1965 and 1966itis mattor operaled n

Sundls, NML as SNARE, Prior to Lhat time

in wat oporated af DeL as that Shiold Tegt

Atrorm inucles.

Propulsion Progerm from 1959 vo 1962 I

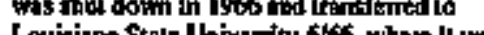

neror sesembled. Ownert Phillips Pelroleum Co.

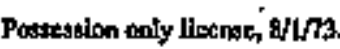

The Neulcon Rutiogneply Fucilly wal uted

to perfonm aerifon raftography of roactor foel

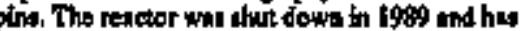

bean converted to a fuel dortoinc bastin.

Owner: Spuce Resintion lebonitory. Thit

TRIGA reaclor was capable or bejng pilied

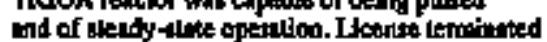

Gill mid-1967 FRAN wat operaled by

LLNL at the Nerwa Tan Silo, and uall

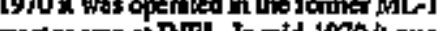

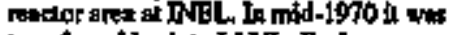

inastemed back to LINL. Fuol was itaroved for prosestitis. 


\section{TEST, RESEARCH, AND UNIVERSITY REACTORS}

\section{General Research (Continued)}

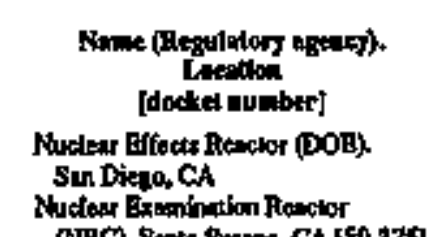

\begin{tabular}{|c|c|c|c|c|c|c|}
\hline $\begin{array}{l}\text { Power } \\
\text { capactif } \\
\text { nek } k W(0)\end{array}$ & 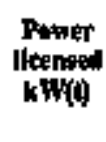 & 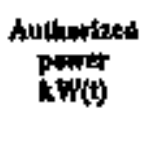 & $\begin{array}{l}\text { Pumer } \\
\text { nd cepecty } \\
\text { net } M \text { (e) }\end{array}$ & 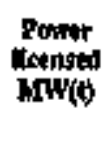 & 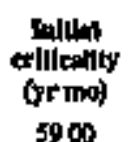 & $\begin{array}{l}\text { Shlow } \\
\text { dom } \\
\text { (5mo) } \\
6400\end{array}$ \\
\hline & & 30 & & & 5200 & $\triangle D O B$ \\
\hline
\end{tabular}

Ouk Ridje Grapthe Reacter

(DOE). Osk Ridge, TN

Prwiling Reternch Reacior

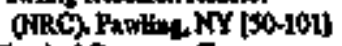

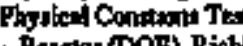

- Hosctor (DOB). Richlent, VA

Rativiton Effects Reactor

(NRC). Duwsogtille, GA [50-172]

3,500:0

3,006:0

1,00000

$1,500,0$

500.0

$1,000.0$

(DOA), Ouk Ridat, TN

Tawer Stielding Reactor

No. If (DOL) Dak Rutar TN

Trmithal Reactor Ten Fuelliy (DOB).

INELSILL, ID

UTR That Reator (NRC)

Mounkin View, CA

Wetinghonet Nacteir Tratuing

Center (NRC).Zion, II, [50 87]

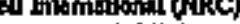

\section{PART I CNIEIAN REACTORS (DOMESTIC)}

$4300 \quad 6300$

58007100

$5500 \quad 7200$

590000

390000 lockhed

PNL

5800740

61006700

AI

SPR. Pritipa bund.

Sindat

STIR. Pool. A

$5500 \quad 200$ TTR-2. GTtphite

PMD.

IRIGA-Min II. 10+2

hydidts. Orwner

TSR, ASRtype in

tont. ORR,

TER-2. Luth

TFRAT.

$5900 \quad 9409$

61006300

7200
TEkAT.

No Dose. Boriphitior

WNIR. Tink Wert.
Cormanent

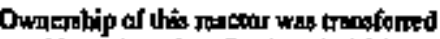

to North Amoricen Rookwell 12fit and wi redesignated the Nucloar Examficullon Rosator tor L 85 rallher thes AB-6. The AB-6, low dedgnuled WHins, was buil and Jima opetated II Downey, CA.II twil rooted io Stut Susma in 1956. licerno tempinated 49887.

Owarr: Lhiter Nuaterar Corp, Licente Lembinued Gnstis.

The RER was previoosly wiod in the vorninued Aircint Nucker Propulion Profram. A lkense andhortzing Lockhted

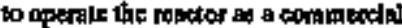

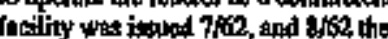
USAF inotiond tho facility to the

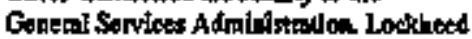
acquired the tuld to tha factily 3,65 . Licterse leminated assm.

Lfento homnintied $2 / 1$ L/82.

Thit westor was previoudy doutgnulod \$TCP Ior SNAF Sticida Twat Rádility.

Omer Gerongl Alonic Lanse tombuled intorts.

Shux down-To be defineled.

Anothorized powre, at, Timatent RX.

Onmer: Americen Rationat \& Simfingd Sinktary Corp.

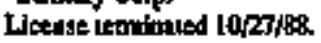




\section{E. Unlversity Feseerch and Teaching}

\begin{tabular}{|c|c|c|c|c|c|}
\hline 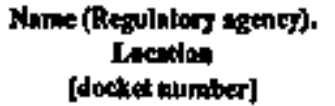 & $\begin{array}{l}\text { Power } \\
\text { copudty } \\
\text { net inW(e) }\end{array}$ & $\begin{array}{l}\text { Power } \\
\text { Jocened } \\
\text { kwigl }\end{array}$ & $\begin{array}{l}\text { Aulhariad } \\
\text { ponwar } \\
\text { kw(o) }\end{array}$ & 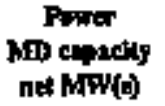 & $\begin{array}{l}\text { Powar } \\
\text { Jleunsod } \\
\text { Mowey }\end{array}$ \\
\hline
\end{tabular}

Bighard Yorag Uaiversily

(NRC), Prow, UT [5026t]

Cillomia Polytechnio

Sime Untuer:is QRRC.

Sap Luir Obinpo, CA [50-39i]]

Catiomtin, Berteter

Iniwertily of (NRC

Bedraky, CA (50-224]

Cillonto, Los Anpeles.

Ualiwertity of, School of

Enpinooring and Applled

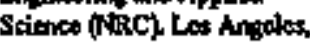

CA [50.5ta]

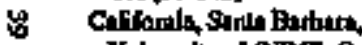

Univenily of (NRC). Sint

Bubura, CA [50-493]

Colholis Uetwentily of

Amerios QRRC.

Wuthing $100, \mathrm{DC}[50-77]$

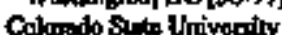

(NRC). Fat Coitin, CO

Cotnotia Untwority (NRC)

New Youk, NY

Delawaro, Uainanity of

(ABC), Rownt, DE [50.98]

Geongin Intidous of

Tectmology (MRC).

Admk, OA [30267]

Kenaus, University of

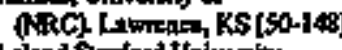

Leland Strutond tontvenity

NRC2 Palo Ako, CA [(6-14)]
1,0000

1000

$5100 \quad 7600$

$6840 \quad 8500$

2500

100

$5900 \quad 74$
Dosingulan.

Trpe, Ptinclpod

inclear

eoptrador

L.7T.

Hombogenocter. AI

AGN401-100.

Hoinog, wotid.

RIGA-Mak III.

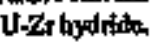

OA

Bdwewce.

Oraphing wallon.

Axpr

$7400 \quad 8600 \quad$ L.77.

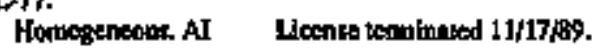

57 00 8600 AOK-201-10l. DECON approved

Horms solid.

ACN

AON-201-10\%.

Flonos, toliti AON

TRIGA-4k II.

U.Zrhydride.

aA

The Colmmbia Uniwextiky Teroh HK D was

ticented to opertito by NRC. Howewor,

Aly of Now Yotk bes not wilhodzed

operusten. Thetefore Cobumbis Univeresty

has nol procused fuel. The lioeste wat

terminules in 1985.

AGN+201-113. Ueenat 10mfinatiod 2/2679

Honot welld

$\triangle G N$

NON+201+104.

Fiomogr solid.

$\Lambda O N$

ACN-201-104 opented ax the Univorainy

of Akron (Ohlo) from 1957 uatil irand ferned

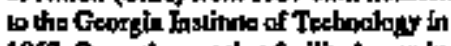

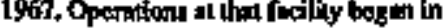

[96. Decorramistionthe of AON-201-108

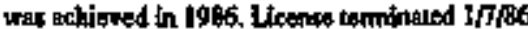

Model 4180.

Paol. BAC

To Deis. Pool

GB

Licror inmonod 10ustigs.

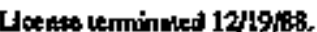




\section{TEST, RESEARCH, AND UNIVERSITY REACTORS}

\section{E. Unlversity Regearch and Teaching (Conilimued)}

\author{
Nine (kpgralutory gyalcy). \\ Locedlon \\ Mempla Sabo Untveraly

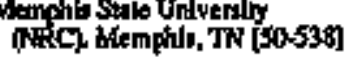 \\ Mictigan Sture Udwenky
}

(NRC) Eat linsing, MI (50.294)

conpardy

Planer.

Authorbo:

Misainstiph Stuk Uniweroidy

(NRC), Sume Collogo, if [50-0]

Novadm, Univanily of (NRC)

Rean NV [ 50.203$]$

North Curotin State

thawertity (NaC).

\& Rntigh, NC[50-11t]

(ARC) Lormat, of [5R-112]

Oreson Siate Uhiveretry

(NRC), Corvilis, Of [50-106)

Pedifechnic Irutinge of New Yoik ORCD. New Yosk, $N Y$ [30-216]

Pueno Rico Rtuolear Centes

Puono Rloo Kivelear Conies

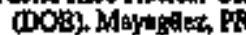

Tetat al Autin, Uniwersily

of (NRC), Aorln, TX [30,192]

Twpkeses Instinto (NRC)

WWo

MowF:

Pawn.

250.0

its

Mintit)

Inliled

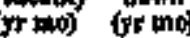

$m 00 \quad 8500$

6900

800

\begin{tabular}{|c|c|c|}
\hline 6360 & 74 的 & LT. \\
\hline 6000 & 7900 & 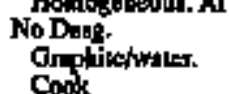 \\
\hline 5800 & 2800 & $\begin{array}{l}\text { MON-2]1-102. } \\
\text { Homos, soltt, } \\
\text { pool, AGN }\end{array}$ \\
\hline 5800 & 7400 & $\begin{array}{l}\text { AGN-201-L14 } \\
\text { BOHOL 10ift. } \\
\text { ACN }\end{array}$ \\
\hline 6700 & 7400 & $\begin{array}{l}\text { AGN-201S-los. } \\
\text { Howos, solld. } \\
\text { AGN }\end{array}$ \\
\hline
\end{tabular}

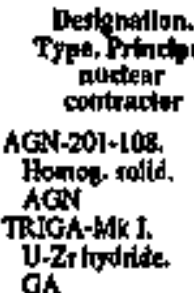

RRR.

Fontonepeous.

Omarish

2,0000

$\mathbf{2 5 0 0}$

$5900 \quad 7900$ L.77.

7007600

TRIOA-FLIP. Pook-TRITA cose $G A$

69009200

TRICA-ME I. tJ-Z/thydride.

$7400 \quad 8412$
OA

AGA-201-102

Howos soitd.

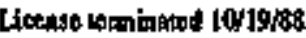

The core of the Michigen Stute Uhtwartily tescter coporiled in the Univarity of nimois

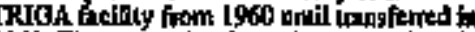
1966. The reactor has been dorommlssionod. The leanse was leminalad 4530 .

Mis Featoe was orjginity openied by Norlh

Carolige Stats University af ollo Raleteh Reagerch

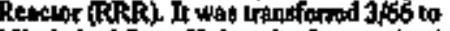

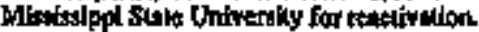
The RRR was ditmentied by NC Stone in 1960. Owlus to fuadints probitom this reteclor was never

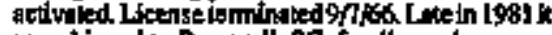

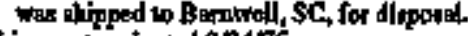
Licease krmitaled 2/24/75.

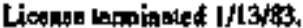

Literse keminatrd 2//4/90.

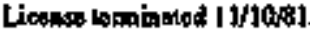

In 1\%57-1962, ACN-201M-[05 wat othacd and operaled by Me Notional Naws hedleal Center, to Now York Uaiveresty sarilyin 1964. A licoule

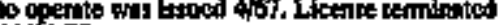
$12 / 21 \mathrm{~m}$.

Owater The Cander for Enviroumanint and

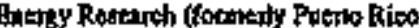
Nockes Center).

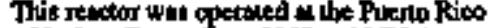
Nuclear Centor trom 1960 to lons it why

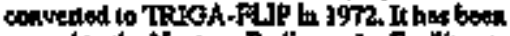

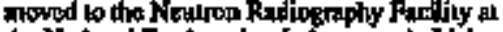
tro Nutional Endintering Laboratory in Idaha. License leminated tori3/s.

AGN-301-102 was opsaled an Okbtonn Stall Univenity, Stllmaler, OX from 1997 mi

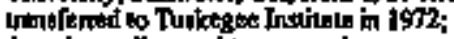
bites is was ficented to opente bin was never sinted up. Tuakegee inktink Itamed ine ficl to the Departunent of Enengy, unit the

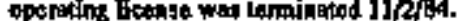


Uwh, Univetiky of (NRC)

Sth Liva Gity, UT [50-72]

Vifinit, Univertly of (NRC)

Chathonerilio, VA [50-396]

\section{Virginta Polyohnte fontiols}

ORRC. Blodshour, VA [50-124]

100.0

Wetinglos, Univerily of (NRC). Sorulo, WA [SD-139]

ImA 0

Wex Vireinla Ualwentity (UARC).

Hogendown, WY [50-129]

Wrilfan Math Rim Uhitwerily (NRC). Heunotil, ']X [50-114

Wyoming Universty of (ARC).

Inninte, WY [S0-122]

\section{MATERIALS PRODUCTION}

\begin{tabular}{|c|c|c|c|}
\hline$\$ \mathbf{m}$ & 9100 & $\begin{array}{l}\text { ACH-20l-107. } \\
\text { Fictinos tolid. }\end{array}$ & DECON approwed. \\
\hline 7400 & 90 & $\begin{array}{l}\text { AGUN } \\
\text { CAYALIER. } \\
\text { Reacioe bypo } \\
\text { not speofifled. } \\
\text { Owner }\end{array}$ & DECON appoved. \\
\hline 5900 & .400 & $\begin{array}{l}\text { UIR-10. } \\
\text { Oruphliefwater. } \\
\text { AS Ing. }\end{array}$ & 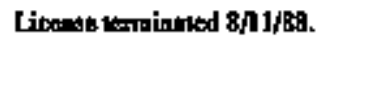 \\
\hline 6100 & 9000 & $\begin{array}{l}\text { Edurator. } \\
\text { Grophilojimilos. } \\
\text { AMP }\end{array}$ & 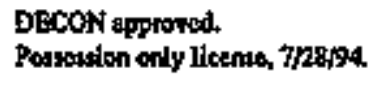 \\
\hline 5900 & 7200 & $\begin{array}{l}\text { AGN-21L-JO3. } \\
\text { Homog, wolid, } \\
\text { pool. AGN }\end{array}$ & Licenst teminaied 9/R84. \\
\hline 590 & 6500 & $\begin{array}{l}\text { ACN-21t-jol, } \\
\text { Homog, wolid, } \\
\text { pool. AGN }\end{array}$ & Lboenis lorminaled 9RSFT. \\
\hline 5900 & 7400 & $\begin{array}{l}\text { L77. } \\
\text { Homgeneoss, AI }\end{array}$ & liconte Iturnindeled $12 / 5 / 75$. \\
\hline
\end{tabular}

\section{PAFT II PRODUCTION REACTORS}

\begin{tabular}{|c|c|c|c|}
\hline Nume (이 semed by DOA) & Datilonaflan & Locellion & $\begin{array}{l}\text { Nudear } \\
\text { dustguer }\end{array}$ \\
\hline 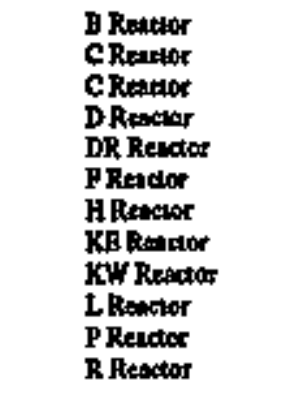 & 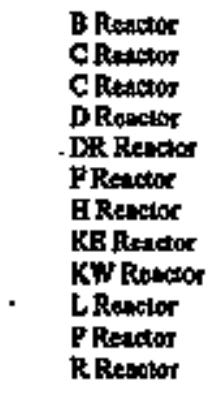 & 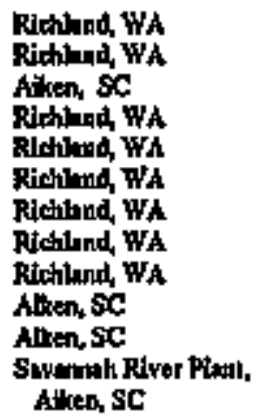 & 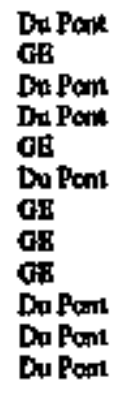 \\
\hline
\end{tabular}

\begin{tabular}{|c|c|c|c|}
\hline$T_{\mathrm{r}}$ & $\begin{array}{l}\text { Aulhorlzed } \\
\text { power } \\
\mathbf{k W ( t )}\end{array}$ & $\begin{array}{c}\text { In'l|a! } \\
\text { exillesilly } \\
\text { Grano) }\end{array}$ & $\begin{array}{c}\text { Shat } \\
\text { (yr mo) }\end{array}$ \\
\hline 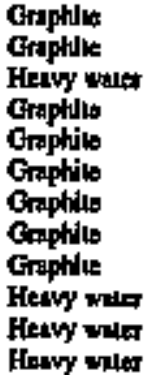 & & $\begin{array}{l}4400 \\
5200 \\
5500 \\
4400 \\
5000 \\
4500 \\
4900 \\
5500 \\
5500 \\
5400 \\
5400 \\
5900\end{array}$ & $\begin{array}{l}6800 \\
6900 \\
9300 \\
6700 \\
6400 \\
6500 \\
6500 \\
7100 \\
7000 \\
9900 \\
9300 \\
6400\end{array}$ \\
\hline
\end{tabular}




\section{PROCESS DEVELOPMENT}

\begin{tabular}{|c|c|c|}
\hline Nome (atl omad by Jos) & Despgantiten & Materitais \\
\hline $\begin{array}{l}\text { Hantord } 305 \text { Tegl Renctor } \\
\text { Lotice Tex Rextor }\end{array}$ & $\begin{array}{l}\text { fTIR } \\
\text { LTR }\end{array}$ & 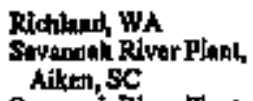 \\
\hline Process Dovelopmient PBo & & 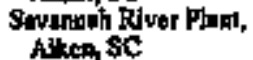 \\
\hline SR 30S-N Teat Pros & Test fille & $\begin{array}{l}\text { Swronmah Rtver Phol, } \\
\text { Aitent, SC }\end{array}$ \\
\hline
\end{tabular}

Sunded Pirof Subeciticel Expedmental Coaplex

\section{PART II PRODUCTION REACTORS}

\begin{tabular}{|c|c|c|c|}
\hline Type & $\begin{array}{c}\text { Authorized } \\
\text { power } \\
\text { kW(0) }\end{array}$ & 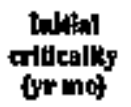 & $\begin{array}{l}\text { Shewl } \\
\text { donm } \\
\text { Grimo }\end{array}$ \\
\hline $\begin{array}{l}\text { Cruphive } \\
\text { Henvy water }\end{array}$ & 10 & $\begin{array}{l}4400 \\
6700\end{array}$ & $\begin{array}{l}7600 \\
7900\end{array}$ \\
\hline Horvy witer & 10 & 5300 & 7900 \\
\hline Gouptive & 1.0 & 5900 & 8300 \\
\hline
\end{tabular}
ratativily offectip of corapootents (ivel lubes,

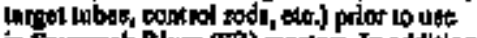

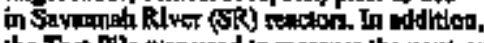
the Teat Pthe wass uned to mensare the neviros abetoption of mistend anowe matcrinils used at

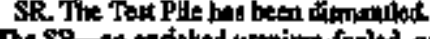

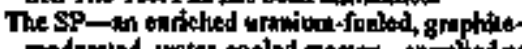

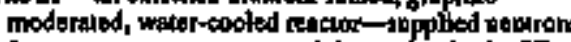
for volioc-component-reactivily testing in the SE, which wat a graphite casmber on rop of the SP.

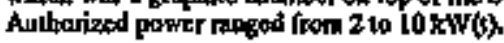

\section{DEFENSE POWER-REACTOR APPLICATIONS}

\section{A. Remote Instalfations}

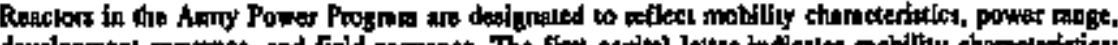

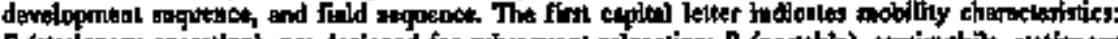

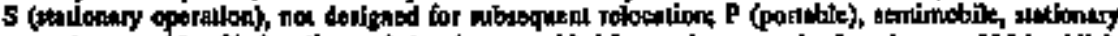

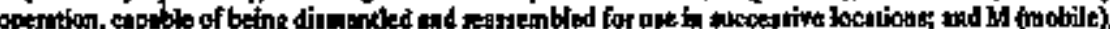

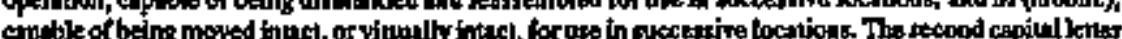

\section{Name (all owned by DOF)}

Lacellon

Fortebto Mediun Fond Plapt.

No. 1, Sumdance, Wr

Ronchbo Hodium Piow or Pint,

No. 3 A. Crmp Contpy,

Gromland

\begin{tabular}{|c|c|c|c|}
\hline Destandion & $\begin{array}{c}\text { Powar } \\
\text { copocity } \\
\text { odkW(o) }\end{array}$ & 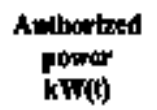 & 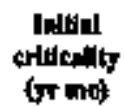 \\
\hline PM-1 & 1,0000 & $9,370.0$ & 6200 \\
\hline MSA & $1,560.0$ & 19,00000 & 6000 \\
\hline
\end{tabular}

PM-2A, 1,50.0, 19,0000
6000

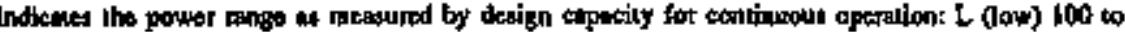

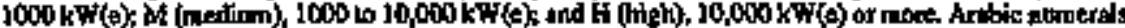

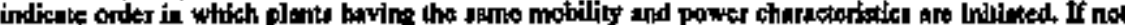

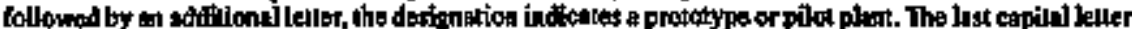

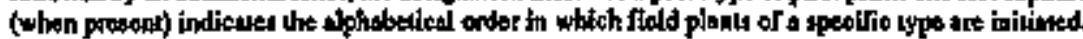

\begin{tabular}{|c|c|}
\hline $\begin{array}{l}\text { shut } \\
\text { domm } \\
\text { Gr me) }\end{array}$ & $\begin{array}{l}\text { Princlatil } \\
\text { mudear } \\
\text { contractor. } \\
\text { Reactor type }\end{array}$ \\
\hline 60 & $\begin{array}{l}\text { Morth. } \\
\text { Prestinni }\end{array}$ \\
\hline $63 \mathrm{mo}$ & Ako, \\
\hline
\end{tabular}

Comont

The PM-2A was abex down T/9/63 mat dlgnentiod

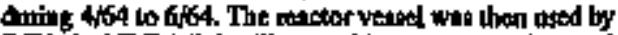

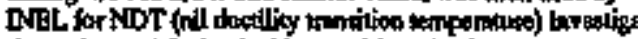

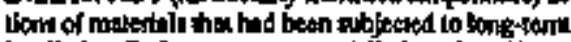

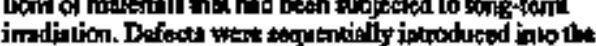

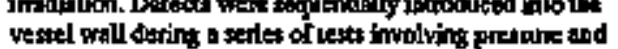

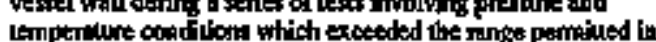

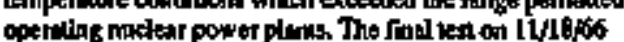

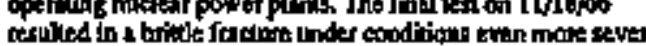

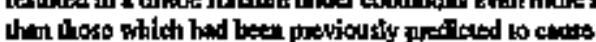

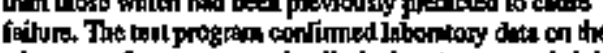

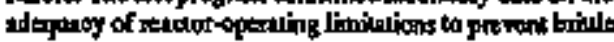
incure of a prespre resel.

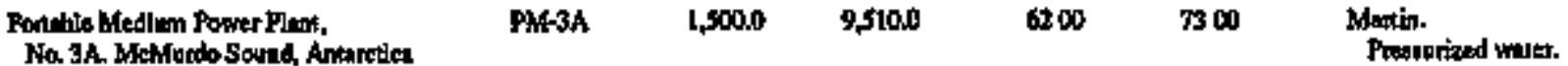




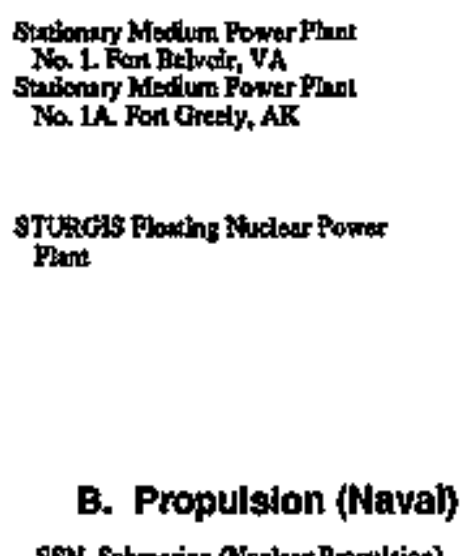

SSN, Sobmaring (Noplant Popullioa).

Namo (all owadod by US. Nayg)

NAUTALUS

SRAWOIF PWR

SHAWOLP Soothm Ravedr

SKATE*

की

SWORDISTH

SARGOH

SPADRAGON"

TrJoN (2R:cion)

HAlJBUT

SCAkA*

SCerriour

SCULPा

shook:

THREXStigR

PERMiT"

PLUR:

BARA"

GEOROS WASHTMGTON"

PATRCK HENRY"

THEODORB AOOSEVELT*

ROBERT E. LEF"

ABRAHAM IINCOLN"

POLIACK*

Back

LACK.

TITOSA*

DAcer

BTHN AL,LEN"

SAM FOUSTON

TFOAAS A BDSON"

JOHN MARSHALL

GUARDFH"

FAASHER*

\begin{tabular}{|c|c|c|c|c|c|}
\hline $\begin{array}{l}\text { SM-1 } \\
\text { SM-1A }\end{array}$ & $\begin{array}{l}1,055,0 \\
t, 650.0\end{array}$ & $\begin{array}{l}10,00000 \\
20,2000\end{array}$ & $\begin{array}{l}5700 \\
600\end{array}$ & $\begin{array}{l}7300 \\
7200\end{array}$ & 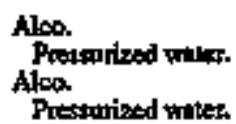 \\
\hline MH-1A & 10,000.0 & 45,00000 & 60 & 7600 & $\begin{array}{l}\text { Montin. } \\
\text { Presarized waler. }\end{array}$ \\
\hline
\end{tabular}

The Amy mado the determinution to slut down the

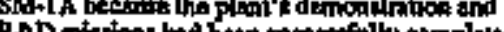

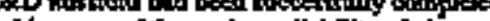
and becanse of the reaty mintilly of cheiper

Tho Mi-1A was inisalled the STYRG (Immerly

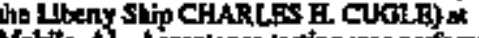

tow

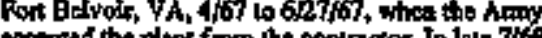

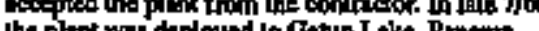

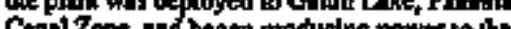

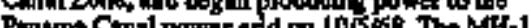

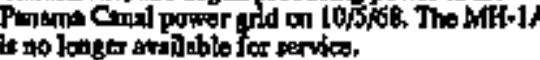

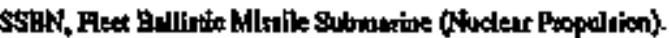

DON, Guided Minla Cruiser ONucloar Propulaion).

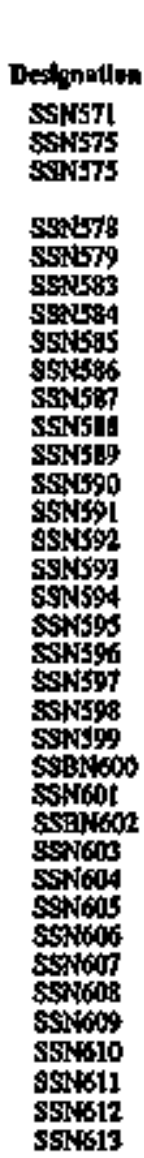

surt-

(بr.to)

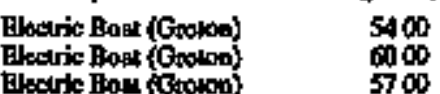

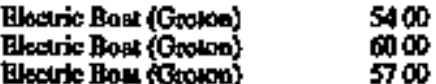

Eledrle Bran (Thown)

Electrio Bont (Grovon)

Partinourh

Hus Istond

ontsinouth

Ectrio Bont (Grotion)

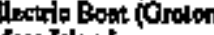

Mate ising

Biectitio Bont (Gromen)

agall:

Newport Ktow1

Pagin

Porlspoult:

Mara biand

Intedla

Clearto Bou (Gronow)

Hectric Boul (Orokm

Eledrio Houl (Grovon)

Arse bind

Elociric Bast (Groton)

Eloemic B

Nys

Prythamowit

Pathemouth

Fint pro

intedicic Bart (Geaton)

Sewpon New

Etectils Bowt (Groton)

Alopont tiews

NYSC

Bectrio Bow (Groven)

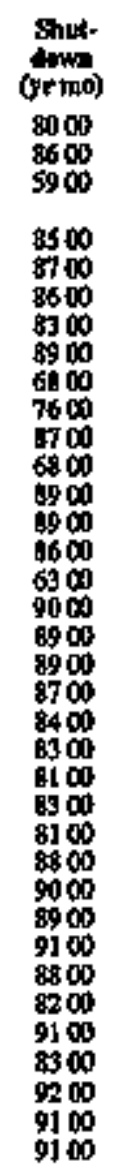

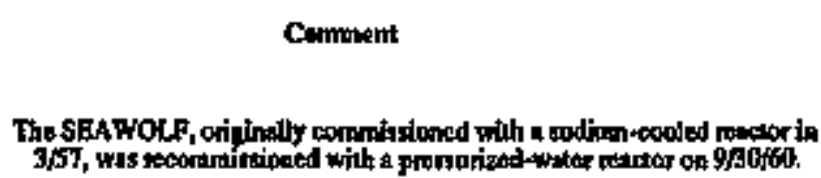

Cenmenent

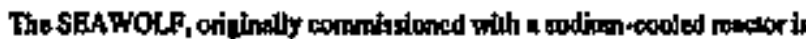

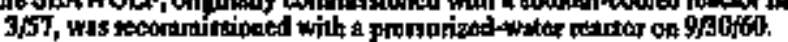

The SCOFPFON wis losk in tho Allartio S/21/36.

The THRESHER was lost in the Athontic 4/10463. 
OMAKA.

CDiconNANI

CoW YORK CITY

Low BeAcH (e patton)

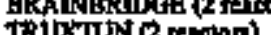

TIX.

THXAS (2 faciont)

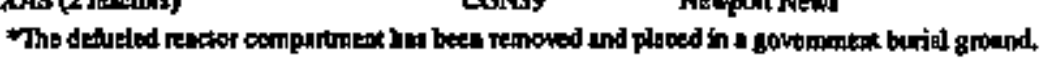

\begin{tabular}{|c|c|c|}
\hline 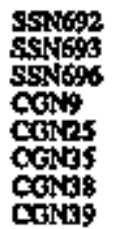 & 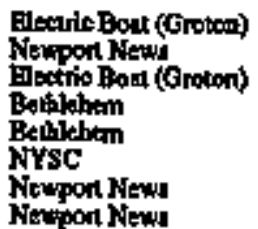 & $\begin{array}{l}700 \\
7700 \\
780 \\
6100 \\
620 \\
7600 \\
7700\end{array}$ \\
\hline
\end{tabular}

$\begin{array}{ll}700 & 9500 \\ 7700 & 9400 \\ 7900 & 9600 \\ 600 & 9400 \\ 6700 & 9400 \\ 7600 & 9400 \\ 7700 & 9300\end{array}$

\section{DEVELOPNENTAL POWER}

\section{A. Electric-Power Experiments and Prototypes}

\begin{tabular}{|c|}
\hline Neme (Opmer). \\
\hline 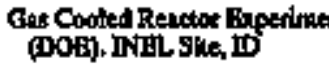 \\
\hline $\begin{array}{l}\text { Matac Low Powtar Finx Na. } \\
\text { (DOB). DIEL, Sine, DD }\end{array}$ \\
\hline
\end{tabular}

Sintionny Low Power Finito. POBH, INEL Stre, ID

\section{B. Propulston Experiments and Prototypes}

\begin{tabular}{|c|}
\hline 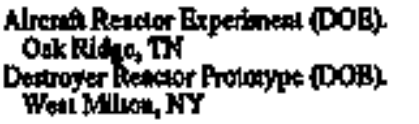 \\
\hline 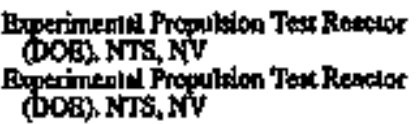 \\
\hline
\end{tabular}

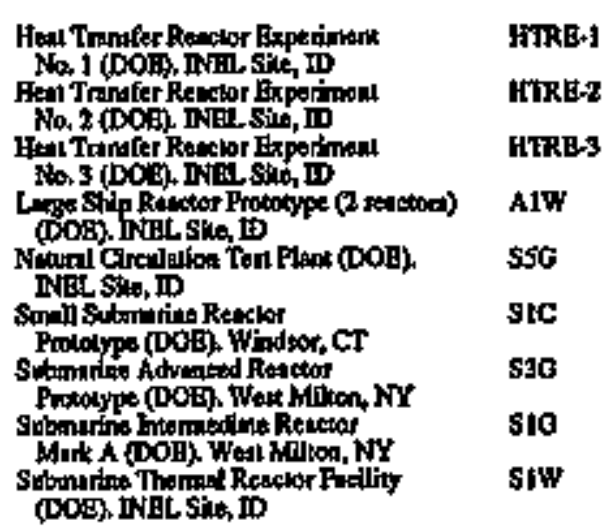




\section{TEST AND RESEARCH}

A. Test

\begin{tabular}{|c|c|}
\hline $\begin{array}{l}\text { Nape (Opmer). } \\
\text { baculton }\end{array}$ & Dedgnaibion \\
\hline 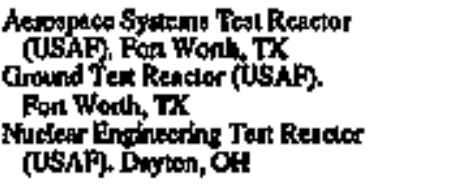 & $\begin{array}{l}\text { ASTR } \\
\text { GTR } \\
\text { NETR }\end{array}$ \\
\hline B. Feseterch & \\
\hline 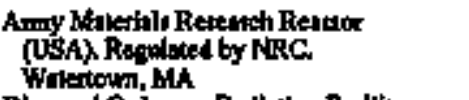 & AMRR \\
\hline 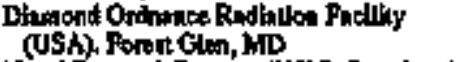 & DORF \\
\hline 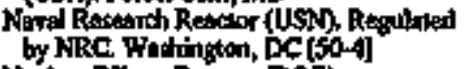 & NRR \\
\hline 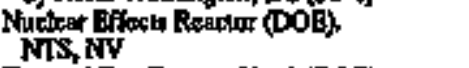 & Spper KUKLA \\
\hline $\begin{array}{l}\text { Therpal Tent Reactar No. } 1 \text { (DOE). } \\
\text { Sthenectody, NY }\end{array}$ & TTR-] \\
\hline 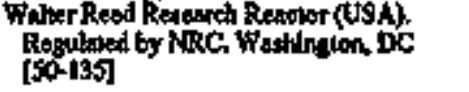 & WRRR \\
\hline
\end{tabular}

\section{POWER REACTORS}

\section{A. CentrakStation Electric Power Plants}

\begin{tabular}{|c|}
\hline 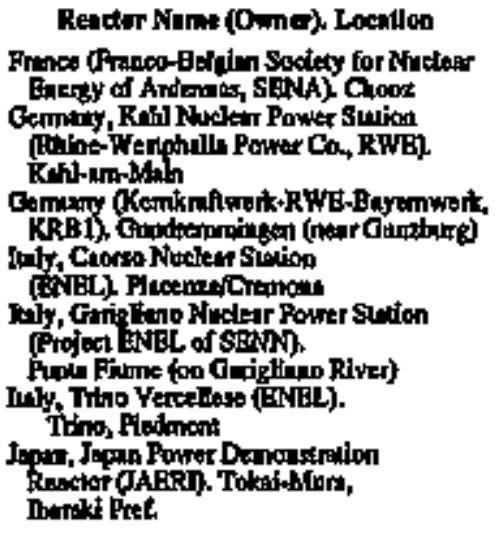 \\
\hline
\end{tabular}

\begin{tabular}{|c|c|}
\hline 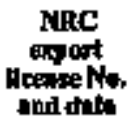 & $\begin{array}{c}\text { Pwinctpal } \\
\text { nucketr } \\
\text { cowractur. } \\
\text { Reactor tope }\end{array}$ \\
\hline $\begin{array}{l}x a+031 \\
0950559\end{array}$ & 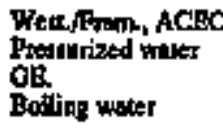 \\
\hline 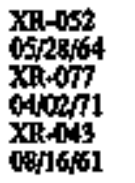 & $\begin{array}{l}\text { GB } \\
\text { Botllog wuler } \\
\text { GE } \\
\text { Boditg waler } \\
\text { GE } \\
\text { Boiling waler }\end{array}$ \\
\hline 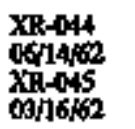 & $\begin{array}{l}\text { West } \\
\text { Prethurited whter } \\
\text { GB. } \\
\text { Botling walls }\end{array}$ \\
\hline
\end{tabular}

\section{PART III INILLITARY REACTORS}

\begin{tabular}{|c|c|c|c|c|c|}
\hline \multirow[t]{4}{*}{ 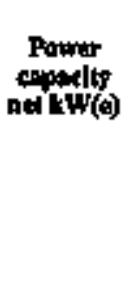 } & $\begin{array}{l}\text { Aclhorlzed } \\
\text { power } \\
\text { ktho( }\end{array}$ & $\begin{array}{c}\text { Inbliat } \\
\text { orlucsiliy } \\
\text { (gr nos) }\end{array}$ & 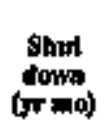 & $\begin{array}{l}\text { Resactor 15pe } \\
\text { Irinclpal } \\
\text { nuclear } \\
\text { contractor }\end{array}$ & \multirow{4}{*}{ 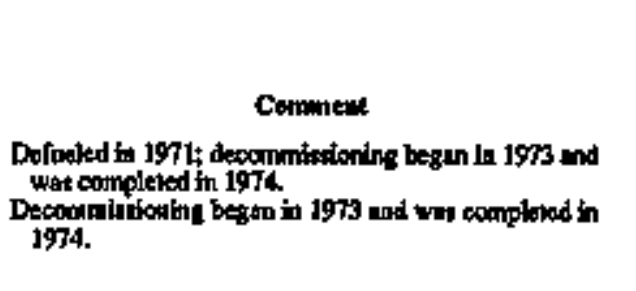 } \\
\hline & 10,0000 & 5400 & 7100 & LWR, Cosyair & \\
\hline & $10,000: 0$ & 5300 & 7300 & Pool Conveir & \\
\hline & 10,0000 & 6500 & 7000 & Tank. Maton-AC & \\
\hline & $5,000.0$ & 6000 & 7000 & Pool BAC & 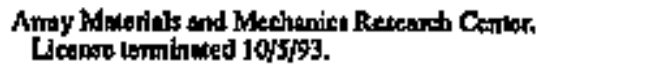 \\
\hline & 250.0 & 6100 & 700 & TRIGA+AKR & Iany Diamond Daboradorios. \\
\hline & 1,0000 & 5600 & 2000 & Pool. NRL, & 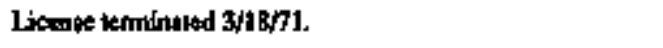 \\
\hline \multirow[t]{3}{*}{$\mathbf{A}$} & & 6400 & 7900 & Pontod bunl. & Standby fuet th storage al OHWL. \\
\hline & 100 & 5100 & 5300 & Gruphitri KAPL & \\
\hline & 500 & 6200 & 7000 & Honoponteun, AI & 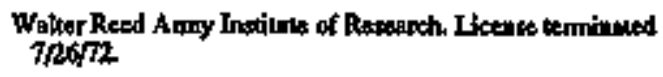 \\
\hline
\end{tabular}

\section{PART IN EXPORT REACTORS}

\begin{tabular}{|c|c|c|c|c|c|}
\hline $\begin{array}{c}\text { Powot } \\
\text { desting } \\
\text { mel hinge }\end{array}$ & $\begin{array}{l}\text { Powts } \\
\text { MW(1) }\end{array}$ & 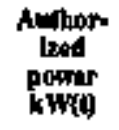 & 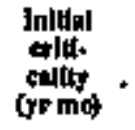 & $\begin{array}{l}\text { Shel- } \\
\text { domin } \\
\text { (trmet }\end{array}$ & Conpinent \\
\hline 305.0 & $1,040,0$ & & 6700 & 9t 10 & Decommisticning is procoses, \\
\hline 15.6 & 60.0 & & 6100 & 1500 & \\
\hline 2970 & $\$ 01.0$ & & 6700 & $\cos$ & \\
\hline 840.0 & 2,6510 & & 790 & trol & Shut down and montlored sinco $\mathrm{J} / 87$. \\
\hline 150,0 & 5060 . & & 6400 & 7808 & \\
\hline 297,0 & 87400 & & 6500 & 8701 & Slivi dows ond nonilopod tinct $1 / 87$. \\
\hline 120 & 9.10 & & 6909 & 801 & \\
\hline
\end{tabular}




\section{CIVILIAN}

Vachily (Repalaws Agews) [dockal number]

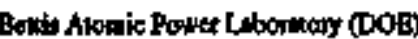

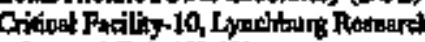
Centher (RTC) [50-13]

Lel Alamot Nalinat Labocalony (DOB)

Los Alemes Notiond Labomiory (DOB)

Onk Ridge Ctkted Engerimealu Faciliky (COOB)

OPWL Pool Cintical Aroterbly, BSF Pool (DOB)

PNL Critic1 Men Lebortiony (DOH)

PNL Criticl Mau latorawy (DOB)

PNL Cridicl Magd Laboratory (DOE

Untied Nockes Comporation, Developanes

Division (ARRC] [50-290]

Tho Potror Fhy ixa Reador

Idwo Division (DOB)

Zewo Power Reector Ho. 6 (DOH)

Zoro Powar Reackor No, \& (DOB)

\section{MILITARY}

Beatis Avonic Pondr Labondory (DOB Heals Atoin's Power Labocalory (DOE)

Kroth Aromilo Power Latormtory (DOE)

Kreils Alonle Power Lobontory (DOH) Kritlls Alontic Power Estormory (DOE)

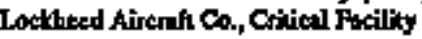
for RER (NRC)

Andear Sufints Fusthy, Rodky Flut

Plant (DOB)

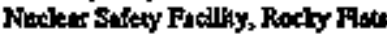

Plint (DOE)
PART V CRITICAL ASSEMELIES

Beptipmont

\begin{tabular}{|c|c|c|c|c|c|c|c|}
\hline \multirow[b]{2}{*}{ Deslgnalin } & \multirow[b]{2}{*}{ Locallon } & \multicolumn{2}{|c|}{ Bepipmont } & & \multirow[b]{2}{*}{ Covment } \\
\hline & & $\begin{array}{c}\text { No. } \\
\text { of } \\
\text { cells }\end{array}$ & $\begin{array}{l}\text { No. } \\
\text { coidrat } \\
\text { pantis } \\
\text { roors }\end{array}$ & Abbrevalon & 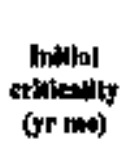 & 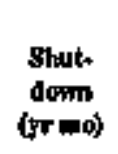 & \\
\hline $\begin{array}{l}\text { LWBCC } \\
\text { SSRP }\end{array}$ & $\begin{array}{l}\text { Pilustrurgh, PA } \\
\text { lynchbuits, VA }\end{array}$ & $\frac{3}{2}$ & 3 & $\begin{array}{l}\text { Betitix } \\
\text { CX-10 }\end{array}$ & $\begin{array}{l}6300 \\
7700\end{array}$ & $\begin{array}{l}8000 \\
800\end{array}$ & $\begin{array}{l}\text { LWB physics. } \\
\text { Close ntonge of apent reacior forl. Licente } \\
\text { teminated } 2 / 88 \text {. }\end{array}$ \\
\hline Parke & 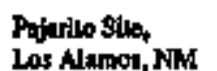 & $\mathbf{g}$ & 3 & LANL, Khm In & 6900 & 8500 & Cold enitieal tor instranentalion lesting Detueled. \\
\hline Veint: & 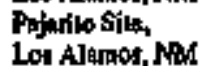 & $\mathbf{I}$ & $\mathbf{t}$ & LANt, Ktrat I & 7600 & 8800 & Ventical babs arsentily mandinus. \\
\hline $\cos$ & Gel Ridge TW & $\mathbf{t}$ & l & OACEF & 5000 & 9200 & Swat down-Defiveded. \\
\hline PCA & Oak Ridga, TN & $\mathbf{E}$ & $\mathbf{J}$ & OANLACA & 5900 & 9200 & Shul down-To be defiolod. \\
\hline EEAS & Jantord Sile, wh & $\mathbf{t}$ & 1 & PNLCAt & 7600 & 9000 & 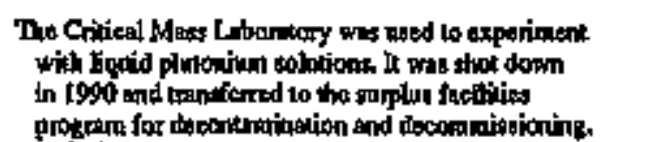 \\
\hline $\begin{array}{l}\text { Hotizonal } \\
\text { RSTM } \\
\text { PTR }\end{array}$ & $\begin{array}{l}\text { Hendord Site, WA } \\
\text { Henford Sitc, WA } \\
\text { Panving, NY }\end{array}$ & $\begin{array}{l}1 \\
1 \\
4\end{array}$ & $\begin{array}{l}1 \\
1 \\
3\end{array}$ & $\begin{array}{l}\text { PNL-CML } \\
\text { PNL-CML } \\
\text { UNC }\end{array}$ & $\begin{array}{l}6100 \\
6300 \\
6700\end{array}$ & $\begin{array}{l}9000 \\
9000 \\
7200\end{array}$ & 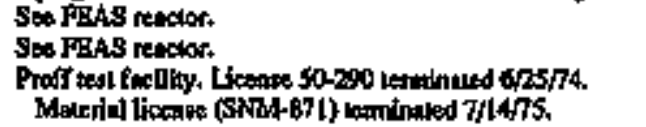 \\
\hline ZPPR & WALt Sins, ID & $\mathbf{t}$ & $\mathbf{3}$ & ANL TDAFO & 6900 & 940 & \\
\hline $\begin{array}{l}\text { ZFiR-6 } \\
\text { ZRR-9 }\end{array}$ & $\begin{array}{l}\text { Artanno, n } \\
\text { Algonit, }\end{array}$ & $\begin{array}{l}2 \\
2\end{array}$ & 2 & $\begin{array}{l}\text { ANL } \\
\text { ANL. }\end{array}$ & $\begin{array}{l}6900 \\
6700\end{array}$ & $\begin{array}{l}8207 \\
8200\end{array}$ & 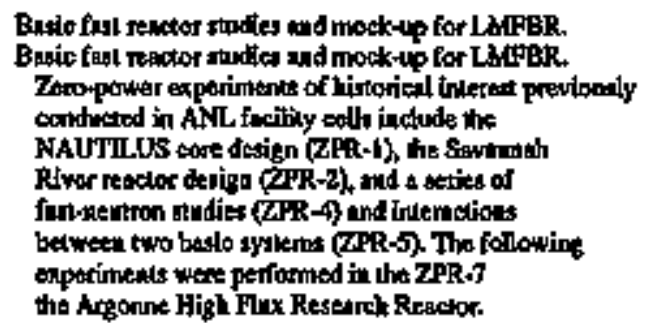 \\
\hline
\end{tabular}

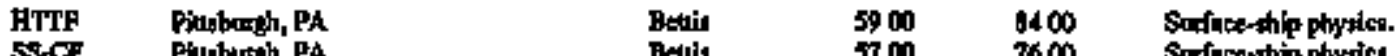

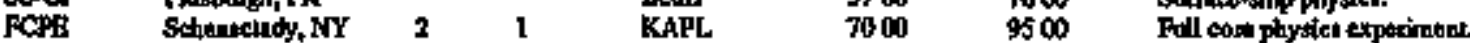

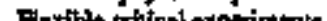

growore phyles wad mock-yp.

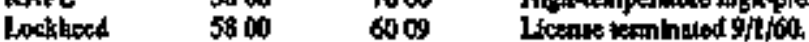

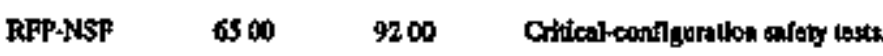

Ventialf Golden, $C 0$

Sylar Table

Wher

RFP-WSF

6500

920

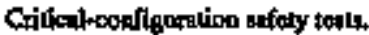




\section{REACTOR INDEX}


AtW, 45

ABRALAM IRYOOLN

CVNT2 (2 ratetorn), 13

SSBN600 45

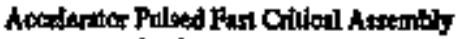

AFPA-II, 35

ACF Indapditin, ho, Manchuserta Intilule of Technotozy, 9

ACPR (Anmular Core Ruthed Rtatior), 35

ACRR (Anmulur Cone Rnsteariti Resutor), B

Advenced Bnergy Resemen Inatiule, Kore.

XR-027, 20

$\mathrm{XR}+073,20$

Alwaed Rectivity Manunement Paoillyy ARMP, 7

Adruced Tou Renton, ATR, 6

Adumoed Tex Restotor Cípital Fucility A'JRC, 2

Aerojet-Gannal Compontion

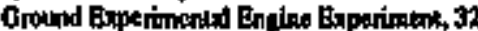

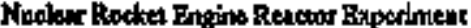
(NERVA), 32

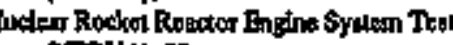
(NERVA), 33

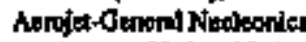

A frante Notional Laboratory, 35

Collforla Polyiechrola Bunte Unhwerily. 39

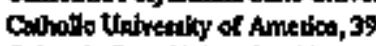

Colondo Stove tiniventy, 39

Delawate, U/tipusitsy of, 39

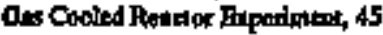

Geocein hotitutes of Tecianology, 39

Who sinte Untwersty, 8

kaly, Univertity or Balemo, 22

Xore, Untveniky of Kyong Hies, 22

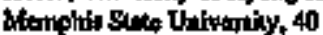

Mobile Low Power Plant Na. 1,45

Stew Mintoo, Univertity of,

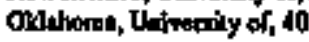

Orogen Suw Univorily, of

Polyodinlo Inotituce of iket York, 40

Swizerhand

Baved, Unlwanity of, 22

Genow, Unherstly of, 47

Teces A A M Untwonlty, 9

Trekesec Intinater, 40

Und, Un'rertily of , A1

Wost Vigialin Uniwenthy, 4

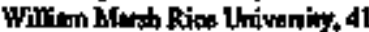

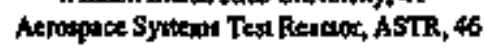

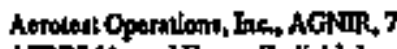

AFRT (Armed Fosece Redioblologs Resend 3pnitulas, 15

APSR (Arganne Fast Somice Reachor), 35

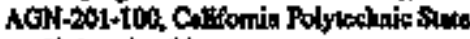

Uabers'ly, 39

A0N-201-101, Cuhollo Untreasty of Ametica, 39

AGN-201-108, Tutkese Instimbe, 40

ACN-201-104, Goorgat Inutiunte of Tochaology, 39

ACN-201-107, Utah, Untwechly of, 41

AON-201-108

Argorint Netional Laboralory, 35

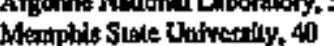

AON-201-109, Colorado Steld Univertity, 39

AClN-201-133, Ditownre, Jniversity of, 39

A(1)-201-114, Ortgon Stan Utiren'ly, 40

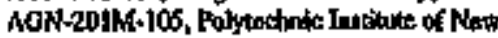
Yosk, 40

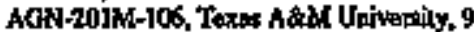

ACAN-201M-112, New Morico, trivenihy of, 9

AGN1201P-103, IS:ho Sicle Uubertily, 6

AON-211-101, Wilim Bintl Rice Unhivenily. 41

AGN-211-102, Okbluonk, tinivaniy of, 40

AON-211-103, Wert Vhrinis Untwentiry, 41

AONIIR 7

Airen'n Rowetor Expetiment, ARB, 45

ALABAMA, SSBNT31, 12

ALASKA, SSBN732, 12

Albomin, Tiann, XR-154, 2

ALAANY, SSNT53, 13

A. EUQUERQUE, SSN706, 12

Aloo Produls ha.

Portstile Medinm Power Flun, Na, 2A, 42

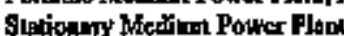

No, 1,43

No. $1,4,4$

ALEXANDIR HAMILTON, SSBAN17, 44

ALEX, NDRLA, SSN75T, I3

Allin-Gialmen Mfr. $\mathrm{Ca}$.

Blok River Re:etor, 27

Bxperintents Ga Cooled Resclor, 30

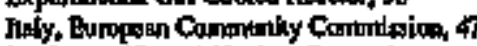

L. Crows (Oenas) Noctar Gemanting Strition, 27

Nockar Fistincering Text Rewaxor, 46

Pauhinder Alomic Piual, 27

South Afiten, Sararit, 19

Sweden, 19
Almune

Jail 1, XR-089, 17

Unil 2, XR-08:, 17

ALRR (Ames Lnboralony Rourarch Reactor) 34

Awin W. Vogule Nueclear Mapal

tintt $]_{\text {. }}$.

Uàt 2,1

Ammoricea Rutiatiox

Auslinia, Monda, 19

lapan, 22

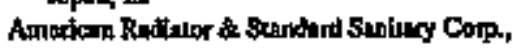
UTR Trat Rescior, 35

Atmexicen SAudard Ino, UTR-1, 35

Ants Laboratory Resenrath Resctor, ALRR, 34

AhF Akontiss, In

Ames Labordory Rereurch Reacior, 34

Antria, Ature 19

Besuello Menoral Intilute, 36

Colifomit, los Angoles, 99

Canda, 2]

Gabidiem, Ino. Resctor, 34

Germsuy, FPeM Garscking, 21

Greece, Denocrilos, 20

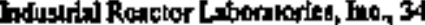

Inot Uhiventily of Tehrich 21

hrecl, Atormic Euergy Commission, 20

Jaly

Gitucit, 47

Silogigia, 4

Japan, JRR-2, 19

Marhattan Colinge, 9

Notheriments, 22

Sonh Caralina Stak Univorily, 9

Pasiotan, PARR, 20

Portugat, RP.1, 20

Suto Univerity of Now Yode, 9

Turkey, 2:

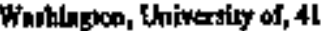

AMITAS, Betgonn, BR-3, Mol, XR-124, 47

AMRR (Amry Materials Reterthe Rescbor), 46

ANDREW JACKSON, SSBN6t9, 4

ANNAPOLE, SSKYHSO, 13

Anmoler Cort Pulsed Reactor, ACPR, 35

Annular Cors Researal Reactor o

APFA-IIT (Accelemor Pulved Far Critletel Astembly), 35

APRP (AmLy Polso Radilmion Fecilily), 15

ARCEETRELS, SNG II
ARE, 45

AFonumL (CP-1]), 35

Arzonns CP-3, retuili a CP-3', 35

AFonne Fal Soumse Reweloc, AFsR, 35

Argonun Low Power Resentch Rewelor.

Jug estamnt, 35

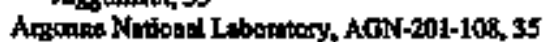

Argoane Hodeur Astonbly for Utiwedily

Thining, Argontal (CP-1I), 35

Amonod Tanmul Sow Re Reactor, ATSR, 35

Atrzons Pablie Servios Co.

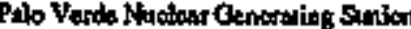

Unth 1, 3

tinin 2,3

Unit 3, 3

Artzonat Univertlyy of, 8

ARKANSAS (2 RaAlon), CoNA1, IJ

Athanua Nuckar Oxe

Unis 1, 1

thisitis

Amed Fencen Radiobiology Rogearch Inalinere, DNA, AFRT, is

ARMF (Aduanced Reactivily Mewarement

Faroltiky 7

Ammour Reveunch Poondation, mincia Inttiule of Teelmology Revesth hathutio, In

Amy Moterialt und hechanica Reasurch Conter, 16.

Amny Malertalu Reverom Reaclor, AMRR, 46

Anny Polso Radiction Prelliny, APRP, 15

ARR (l-54) (hrowor Restanth Reactor), 37

AS Inc.

Amorican Sixndard Ino, 35

iown Stoto Univerily,

United $\mathrm{K}$ :igdom, A?

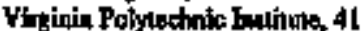

ASHEVItLIE, SSNTSt, Is

ASHRO, SSN6AB, 44

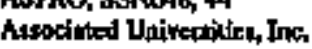

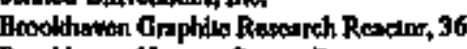

Brooldamon Neulron Source Reactor

No. 1,36

No. 236

Atucintion for Radlation Resenrch, Gommany. $X R-075,47$

ASIR (herowate Syalents Tent Reenor), 46

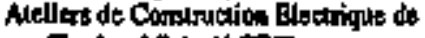

G) mitaros S.A. (ACBC)

Belglim, Thange, Und 1, is

Ictinco, 46

ATLANTA, SSTVII, I2 


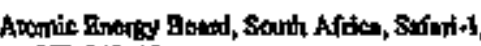
XR-042, 19

Atomito Intemalion:

Alowited Imemulionts, 36

Berli, Cxy of, 47

Brighans Yourg Ualyonily, 39

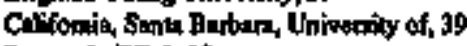

Denmatik, DR-1, 19

Buprimentel Ongapia Cooled Resesp, 30

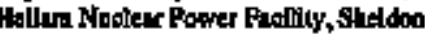

$$
\text { Stration, } 27
$$

Thoil Bntilme of Tredinolosy Restarch Insaltutio, 37

Ialy, it

Jong, 4

Xinetio Broseiment on Waler Bolitert, 37

Vivtruore Wist Botter, 3 ?

Neviday Utulwerahy of, 40

Nucilear Ex mationion Reachor, 38

Onjenls Moderated Renclor Bxperimenl, 91

Pique Nitclear Potwor Farilíy, 28

PuonoRlco Necker Conles, 40

Rocktell Intertetionen, 31

Shistd Toxt and Iradtalion Reactor 38

SNAP-GR Davdopmtarlol Sythen, 31

SNAP-02 Brperimetitel Re:cior, 3]

SNAAP-OS Devetopmantri Reattor, 31

SNAP-08 Exporimental Reactor, 31

StAAP-10A Flight Sygton

$$
\text { s10.5 }-4,31
$$

S10RS-5, 32

SNAP-10A Flight Symen Ground Tes No. 1, 32 Na 3,37

SNAP-1BA Thenteen Tesl Na, 3, 36

Sodthm Reacior Buporinen, 31

Weluer Reed Resturel Rewetor, 4

Wyoriog, Univenity of, 41

ATR (Advinced Teat Reteloc), 6

ATRC (Adrunoed Tetr Reackor Critleal Fuchily),

ATSR (Mcosane Thom it Source Reatior), 35

AUCUSTA, SSNT10, 12

Anterilia, Monu, XR-099, 19

Auntrit

Atran, XR-02s, 19

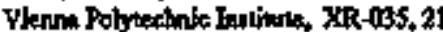

B Rearbor, Ridilind, 41

Buboock \& Wiloos Co

Antinges Nuctetr One, Unit 1, 1
Bobeod \& Willoor Ca, (Continded)

Bellefonta Nuoker Plank

Uant 1,5

$$
\text { Uall 2,5 }
$$

Bratil, Uaiveniky of Swo Pasla, 2 )

Ciynul River Nhaloar PJant, Unk 3, 2

Denti-Bene Nucketr Power Stalion, Unit 1, 2

Gemany. FRor-1, A7

Inition Po'nt Stallow, Unil 1,27

Inly. Cemer for Miliury Appilestion of Nudear Energy, of ?

Lymathoust Jool Reoctor, LRP, 36

Michigan, Uhivently of (Ford Nucket Retatoj) 9

Nucter Dewelopananl Conter Ton Renctor, 34

Oeonee Nuckenr Sation

bitt 1,3

Unk 2,3

Un' 9,9

Runtho Seco Nudenr Ceatraing Station. Uhit 1, 28

Throe diflo hind Nucclone Stotion Whit I, S

Uhik 2,28

Virginis, Universtly of, 10

Washinaton Nooket Pmizer, Unit 1,5

BAINERIDGE (2 mactort), OTN25, 45

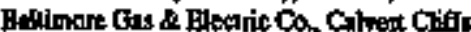

Nuder Powat Plont

Un'l 1, 1

Unit 2, 1

ALLTD4OAR, SStrio4, 12

Hendedesh, hitintes of Nodoar Technotosy. XR-125, 19

EARB, SSNF56, 43

Hesed, Unlveniry of, Swhtzorland, 2

BATFSH, 5 W681, 11

BATON ROUUGE, SSNE89, 44

Butelle Manorial Intilute, BRR, 36

BAWTR (Eabeock \& Wikg)x Teit Renctor), 34

Besvor Valley Power Sumion

Jaht L, I

Uais 2

Belying

Ex-3, Mol of

Doel

Unik $t, 15$

Uni 2 , Is

Uhik 4, is
Bewer Yalley Power Siuloe (Conimued) Thange

Unik I, Is

gellefoale Noctear Pian:

Uhis 1,5

Lnit 2,5

Bendix Aviution Corp.

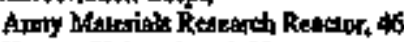
Kunsts, University of, 39

BENUAMTIN FRANKIIN, SSEN640, 4A

BEROALL, SSNG67, 44

Berlin, CKy of, XR-A12, 47

Bemitche Kratwelke AG, Swilzedard, Moldeberg, XR-06t, I

Bedtehen Sxecl Co, Shipbailding Divition BAINRRIDCE (2 nictors), 45 LONG BEACH' (2 reactors), 4

Benti Atonic Rower Laboratory

HTTF, 48

LWBCC, 48

Btc Rock Point Mudes Plant,

Bto Tw, 22

BiL.FISH, SSN676, 11

BIRMIINGHAM, SSNVSSS, 12

BLUEसISB, SSN675, 44

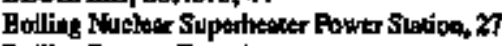

Bolling Rejcter Experiment

DORAX $-2,-3,4,29$

No. I, BORAX-1, 29

ISE, senti, 13

BORAX R actore 2A

Bosion Editer Co. Pilgrin Nocteen Parrer

sution, Uaid to 4

BCSTON, SSN703, 12

Brituinood Sisulon

Unil 1. 1

Unt 2

Bagat

Anari 1, Condrid Electricta do Furdas, $\mathrm{XR}-08 \mathrm{~s}, 15$

- Mriss Geril, Univertity of, 2 Sus Prilo, Univerrity of, 2

EREMLIRTON, SSNG9E, I

Brigh:m Youns Uniweniky, 1-77, 39

Beookhaven Graphtico Research Rewtor, 36

Bnotblaver Metkeal Raventeh Reaclot, 7

Erootbarved Nationtal Laboinany

로 Nebiren Sounce Reiclor, 36

IFth Fux Betat Reculor, 7

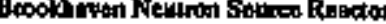
No. 1, SCHTO0, 30

No. 2, PHREISIC, 35

Bown Boverilk roop, Gemana, XR-AKR, 4

Browns Berry Nooletri Powtr Simion

Unil 1, 1

Unil 2, ]

Unlt 3.

GRR (Ealtello Retears Rector), 36

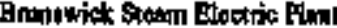

Unit 1, 1

bing

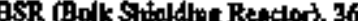

BUTHALO, SST715, I2

Buftalo Materiula Retenth Conke, State Uaiveraly of New Yort, 9

Bubk Shielding Retctor, BSR, 36

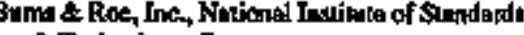
\& Tecturology, 7

Bymot Station

Unin 1, 1

Uuh 2 i

CReackar

Aken, 41

Nochinot, 4

C.H.,., Mnemora, Marocto, XR-158, 21

Callformb

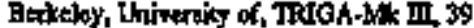

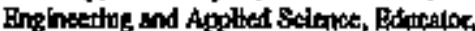
39

Ifvine, Univensily of, TRTG A +MI I, 8

Los Anpoles, Untvartity of, Solvool of Santa Bntara, Uhituertily of, L-77, 3

CALIFORNLA (2 reacton), CON36, 13

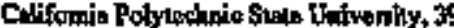

Crtifomia Potylectrio Siato Uhivenhy. ACR -201-100, 30

Crinum Plwk, Un' 1, 1

Cotvent Chifis Nocketr Power Ptan Uail 1, ]

Unit2 1

Canda, Mabdater Untverdty, XR-011, 21

CARL VINSON (2 matort), CVNTO 13

Conolian Pown \& 1 toht $C_{3}$

Brudwitek Stean Eloctric Fint

Unit 1, 1

Unit 2 ,

H.B. Robtonon Plan, Unit 2,

Sheumon Hintris Nodour Rower Planh. Unit 1,4 
Curolinn-Virgetrit Nocker Power Aspochect,

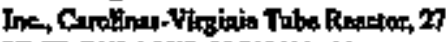
CASRIII PULASKI, SSBNOES, 4

Colawbo Noclear Station

Un'll 1, 1

Unil 2, 2

Cavolic Univerily of Anertica, AOrt2Jl-101.

\section{CAVALIFR 41}

CAVALLA, SST68\%, 11

CFF(Crilleal Hopetimeall Fuellity), 48

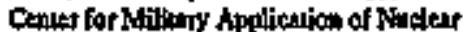
Finents, July, XR-0e6, 47

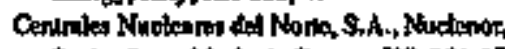
Spuln, Sinte Marta de Gavon, X月-064, 17 CERP (Crilloal Expedimedil Rerarch Paotiny,

Loctheat Airerfit), dt.

Corkup (Coupled Futt Rewatohy Mounuranent Fabliky) $?$

CHARLOTTE, SENTE6, I3

CHEVENNE, SSFV73, 13

CEICAG0, 55Nm21, 12

3 Chicugo PIo 1, terill es CP-2,36

Oisugo Pile Na, 5, CP-5, 34

China, Repablin of, XR -020, 21

CINCLNNATJ, 35N693, 45

Cindiden, inc. Revar, CIMR, 34

GTY OR CORPUS CIRISI, SSNTS, 12

Chanenina, 37

Cloveland Btectite Mouninutiog $C_{0}$

Penty Luckear Powar Ploot

U.h 1, 4

1hin 25

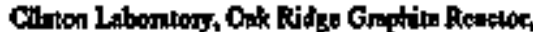

Cinon Pownor Sinton, Unat 1,2

Cotombio, LNAR1, XR-053, 19

Colocado Stm Unlventhy, A(TN-20I-109, 39

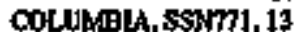

Colombin Uhiverrity, TRICA-MK II, 39

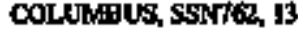

Comancho Peak Steant Eletrile Stalkan

Unil-1, 2

thit 2

Combrialon Engtrateing loc.

Arkanan Mudour One, Unat 2,1

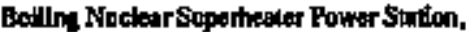

Conbualoc Fingineeding Iner (Contraed)

Calver Cliffs Wpoken Power Phnl thinh 1, ]

Uhis 21

Font Cathoun Siution, Unit 1, 2

Koren

Udhin 3,18

Ukatin: 4 , I8

Yongewint-3, 18

Yangewang +4 , ]

Yougewent 5 , 19

Yongrwant-6, 18

Mhino Yakee Alomis Powor Plant,

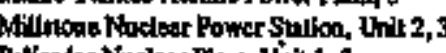

Paliandea Nockonr Pint, Uhti 1,3

Pato Verda Nurlusar Ganemiting Simtion

Unit I, 3

Unit 2,3

Unil 3,3

S. Lucis Pent

Untit 1,4

Unit 2, 1

San Chodfe Kuder Geremting Stwito

Unii 24

Un't 3, 4

Wuled ond Generating Sution, Uril 3,5 Comel, 22

Commontralkal Edison Co

Batidwood Station

Unit 1, I

Unta 2, 1

Byron Sintion

Unill 1, 1

Unil 2, 1

Droitan itucleur Power Simlon

Unil 1,27

Uni12.2

Unta 3,2

Lo Sollo Counly Siction

Unil 1, 3

Unil 2,3

Quadicluse Sintion

Unit 1,4

Unil 2,4

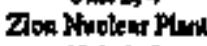

Unir 1,5

Untit 2

CONRECTICUT, SSN22, 16

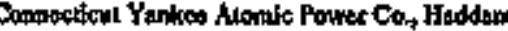

Next Pmin, 2
Convoldated Etison Co. of New Yout, Ine Indin Pain Sirtion

$$
\text { Unit } 1,27
$$

Conmuser Power Co

Bit Rock Poinl Nuclest Pient, 1

Pallandes Noclent PTut, Dhit L,

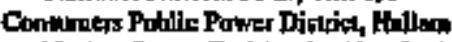

Ninalan Powar Fadilicy, Sheliton Sintion, 27

Conmir Divition, Gentepil Dyamias Cor

hopospoco Syjtom! Teal Resotor, 46

Ground Tou Renction, 46

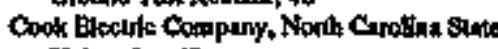
Universily. 40

Cook Nuckow Powrer Plap

tinh 1,2

Unt 2

Cooper Nuclest Sulion, 2

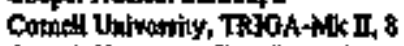

Comets tamonily Zoro Power Renctor, ZPR, E

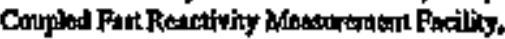
CFRMF, 7

CP-2 (Chicaso Plat), 35

CP-3r:35

CP-5,34

Cthled Expedimenta Feciliky, Ouk Ridge, 4t

Critical Fabiby-10, Linchbers, Rexourch Center. SSRP, 48

Cyyblel River Nurdear PJanl, UnL 3,2

Cumit-Wigln Cotporstion, Mincowi ax Rolla, University of, 9

Cunlewitin Nuoker Reieard Labortany af 1he Conmonwenth of Pennsytvenia, CWRR.

D Reactor, Bichtinnd, 41

DACE, SSNEOT, 43

Daloyland Ponver Coopertitve, La Cromse (Gevou) Nochar Generating Stalian, 27

DAIJAS, SSN760, 12

DANIILL BOONE, SSBN629,44

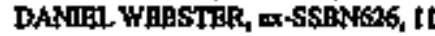

Dacontad, University of Oenminy, 47

Davir-Beano Nuclear Poner Sintion, Un't ], 2

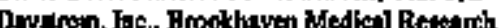
Rowctor, $T$

Desp Sobmetyence Rerench Vethicle, NR-1, 13

Deinwere, Uhivesily of, AGN-20L-1 13, 3 ?

Delife Techaied Unitertily, Nelhatenda, XR-000,
Demknutralinn Reartor, 36

Detrintit:

DR-1, XR-006, 19

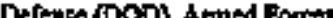

Redioblology Reseursh thitiout 15

Deperment of the Interior, U.S. Geotopicel Survey Lboritory, a

De:iroyer Reactor Protolspt, DtG, 45

Detroit Edison Co., Enico Fenmi Alsmie Powt Plnix, Undi 2, 2

Diable Compros Nueloar Powter Plan:

Unit 1,2

Unit 2,2

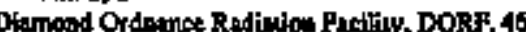

DOB Demonsiralion Reecior, O4l Ridgo, 36

Donald C, Cook Nodear Fower Finn

thit 1.2

Unit 2,2

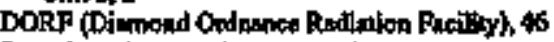

Dow Chemical Ca, 'JPIGA-Mld, 7

DR Restion, Rtalland, 4L

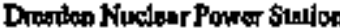

Uhit 1,27

Unil 22

thil 3,2

DRUM, șist, 4

Do Poal de Nemours \& Conpany, 3a, R.I. B Ronetor, 41

CRoatiter, A1

$D$ Rositor, 41

PReactor, 41

Henifed 305 Tetr Reacos 42

Hetvy Want Contponewte Tost Renctor, 30

KRecctor, 10

1. Reacioc, 41

Lalloe Tea Rewior, 42

P Rotetes, 41

Procesa Davaloparant Pato, 12

R Regecot, Al

SR 395-M Ten File, 42

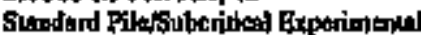
Conjaplax, 42

Dome Amold Eneroy Center, Uall 1,2

Doke Power $\mathrm{Co}$.

Crawbe Nordear Salion

Und 1, 1

Ocones Nuctear Sietion

Unit 1,3

Und 2,3

Unt 3,3 


\section{REACTOR WDEX (Continued)}

Duthe Powrer Ca. (Contiued)

Willon B. MeCriter Nualen Stulow What 1,5

Uhin 2 s

Ducquence Ijghi Co, Onto Ediman Co.

Basver Villoy Powtor Sulloa

Unil 1,1

Unil 2,

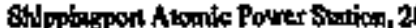

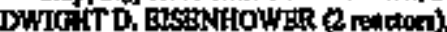
CN469, 19

RAEP, 36

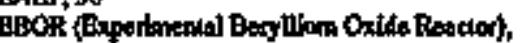
29

BBR-1 (Buperimental Breder Beartor) 30 BeR-II (Exponimontal B resior Reaclor), 30 BBWR (Expertmental Bellins War Resclor), 29 Bdecular

Cilifomla, Lou Angeles, Univora'ky of Ingineering tord Afplled Stionco, 39

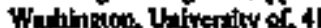

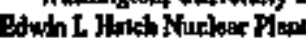

thit 1,2

Unis 2,2

4 EGQG limina, InF

Los of Puld Tan, is

Power-Burnt Fuelliky, 35

EBCR (thopstruental Gat Cootod Resotot), 30

Fik Bwyt Reiclot, 27

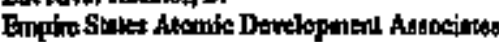
and GE, ESADA Valleciks Exparinamil Sopedieat Retoror, 29

ENFit, Inly

Caran Nocicar Staine, XR-077, 46

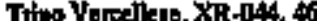

Finergy Comer, Ltelherlonde, XR-017, 19

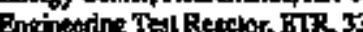

England

inperin Chandical Indutsiot, XR+OR, to Sizowell B, XR-14t, 15

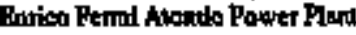

trin 1,27$$
\text { Uath }
$$

Bntergy Opentikus tho.

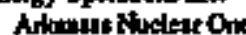

Uhit 1.1

1hail 2,1

Gand Giv thetar Staika, Unil 1, 2

Biret Best Sumion, Utik 1,4

Wakefond Generatiog Staich, Ǵnit 3,5

ENTERPA15B (on recton), CVN65, 13

BOCR, 3D
BsADA Vallectios Bxperimenjul Spopiteat Reeclor, BVESR, 20

ETHAN AZLUEN, SSN604, 4

ETR (Enytucaring Tost Reackoh, 33

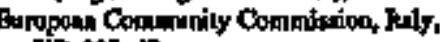
XR $-007,47$

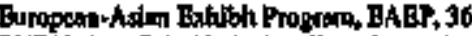

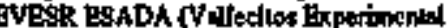

Suptemeal Restor), 29

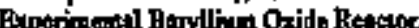
$\mathrm{E}$ EOCOR, 29

Experantend Boiling Woue Reatot, EBWR, 29

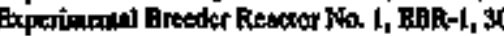
Experínental Broeder Rosator II, EBR-IL 30

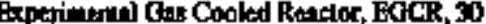

Enporimented Orgugic Cooled Reustoz, BOCR, 30

Experimental Propulsion Teet Reacior TORY IIA, 45

TORY IIC, 45

FRetacir, Richland, 41

Fiarley Nouteat Plant

U. 1 in 13

Unik 2, 3

Pat Burm Renctor Paclliky, PBRF, Is

Fus Fiv Ted Foilly, FrT, 33

Fanl Neutom Soprwe Rractor, BNd/R-1,36

PBRP (Fan Burd Reator Fadiliy), 15

PCPH (Foll Core Fhyales Exporiandi), 4

Funn Atonio Powtr Para

Unh t, 2t?

1hin 2,2

PFIF (Fin Ftox Tert Fuciliky) 33

Fin TTG, Roly, XR-0l6, 48

PTNBACK, SSHSTO, It

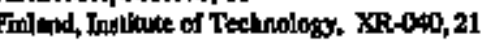

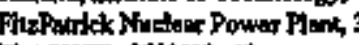

FLASHERR, SSN613, \&3

Flolog, 22

FLONJDA, SSBN728, 12

Fleridh, Univeratty of, UFTR, 8

Poride Poner \& is ist Co.

St Luck Flon

Unit 1. 4

Unil 24

Torkey Poink Piant

Unit 3,5

Unil 4,5

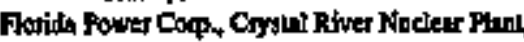
Uhik 3,2

PLYMNG PISH, SSYW673, 44
Pord Nuctear Roactoc, Mistriogn, Untversity of Ford Hacios Reaclor, in

Poot Colkoun Stution, Uhlk 3, ?

Poot St. Yrim Nickear Gencrallag Siclion, 27

Foster Whocter Corp.

Denturk, DR-2, की

Livardon Rod Type Reacer, 37

PPR, 48

Brarentome

Tibtorge, Uhit $I_{2}, 15$

Finkes, 46

ThAN, 3

Frace, Franco-Belgian Sociesy for Nuclear Enerty of Ardennes, SENA, 45

FRANCIS SCOTT KBY, SSBN 657,14

Fronco-Balgan Society for Nucleor Enorgy of Addennes, SEKA, Froes, 46

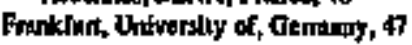

Fukurifma Dai-jebj Power Siation

Unit 1, 17?

tinit 2, 17

tint 6,17

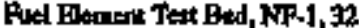

Puel Filemand Test Rentor

Powe-1,32

Peprog-2,32

Ges Cooled Renctor Bxpeximitent, GORE, 45

GATO. SSI'615, 44

Ceneral Aromice Tochnologies, 36

Acceleavor Pulsed Pat Ctilical Alatembly, 35

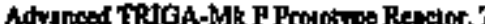
Atrovent Operations, fots,

Abatain, 2 '

Annular Core Polsed Reactor, 35

Atizosten Univertity of, 8

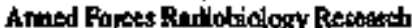
hnilale, DNA, 15

Austit, 2

Bungludeh, 19

Brater 25:

Coniforni

Bokekcy, Jalvertiny of, 39

Ivine, Uhivertily of, 8

Cofumbin Unfueraiky, 39

Cotnell Univeralky, 8

Ditnton: Orduance Radiption Farilky, 46

Dow Comberl Co, 7

Finglined, 19

Exparianenled Beryiliman 0xids Rencios, 29
Geweral Alontion Tectmolasies (Conthuent

Finland, 21

Font St. VIain Nuokear Bancrating S184ion, 27

Demany

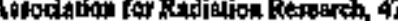

Alunowe Wedicel Collete = m, 21

Ictidelbers, Inathune for Nueles

Motheine, 21

Meinz, Johwites Gulenbars Univantily $\alpha, 21$

IItinals, thiverity of, 8

midontegia

Bendang, 20

lu1y

Yogyateonte, 20

Indifo Atency for New Todmolog:

* Energy und the Enwirenmont, Rome. 20

Japsin

Pria, Uniwertily of, 22

Masuht Cobeto of Technotony, 2 NSRR, 19

Rikkto Univertily, 22

Kanan Sinco Uhivanlay, 9

Korga

TRIGAAMk II, 20

TRIOA-Ak III, 20

Malaysia, 20

Maryland, Univentity of, 9

Moclettrin Naderer Radialion Conker, is

Mexico, Salazar, 20

Michigan Stote Untwertily, 40

Moweco, 21

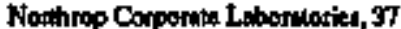

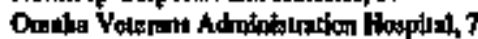

Oretion Stove Uniwersiky, 9

Pach Datom Awomic Pouer Sation, Uhit 1,27

Pern Stere TRITA Reaclot,

Ptriopter, Republis of thb, FRR-1, 20

Puerts Rico Nonbutr Canter, 40

Roed Colloge, 9

Ronntini, 19

Sin Diepo, 'ERIGA-Hk 11, 36

skowenia, 20

Toxill A AMU Univerity, 9

Texas it Austin. Unfwerdty of

TRIOA-Mk J, 40

TRIOA-AMk II, 
Cokend Avaice Totarologles (Continned) Thithend, TRR-1, 21

Turkey, 21

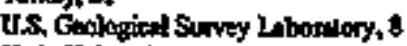

Uhu, Univentiy of, 10

Vietnom, 4t

Wathingos Sute Unirentity, 10

Wreconein, Univertiry of 10

7 Nitro 21

General Dymentes Conp.

Electric Boot Division

ARRAHAM LNCOLN, 43

ALABAMA, 12

AENSKA, 12

ALBUQUERQUE, 12

AIEXANDER HAMILTON, 44

AIBXAFIDLA, IS

ANRAPOUS, 13

ABCHERFISH, 1

AuOtust, 12

BALTMSOtRB, I

BATPSH, il

BUWTAKIN FRANRI.JN, 44
Capap1 D, mamier Corp.

Flectric Bow Divition (Continued) HERRY L STIMSON, 44 HEERY M JACKSON, 12 EYMAN O. AICKOVER, 12 DDAANAPOLS 12 JACKSONVILLE, 12 JAMTS K. POLK, II

KERTUCKY, 12

LAPAYETTB, 4

LA FOALA, 12

LOUDSTNA, 14

iOUISVILt 12

MATHE, 13

UAAYIAND, 12

MANM, 13

MCHIIGAK, 12

OHNNBAPOIJS-SAINT PAOL, 12

NARWHAL 11

NATEAN HAEB, 4A

NAETILUS, 43

NEIBRASTA, 12

NBVADA, 12

NEF YORK CTIY, 45

OHIO, 12

OMHHIA, 45

PARGO, 44

PASADERA, 13

PA'TJCK HENRY, 43

PENWSYLVANIA, 12

PHTLADELPHA, 11

PEOERIX, L2

PTTSBUURGH, 1

PORTSMOUTH, 12

PROVIDENCH, 12

RHHODE ISI,AND, 13

SAN JUAN 13

SANTA FE, 13

SCORPION, 43

SEA HORSB, 44

SEAWOLP, 13

SEAWOLPPWR, 43

SEAWOLP Sodium Reador, 43

SILVERSDES, 44

SKATE, 19

SRJPIACK, 48

SPRANCFIELD, I3

STURGEON, 4

STURGET, 44

TECUMSES, 44

THOHAS A. BOISON, 4
Genetri Drnemis Corp

Flextric Bon Dtwision (Contimued)

TOPEKA, 13

IREPANFG, 11

TRTrow (R Recelon), 4

THIIIEER, 43

ULYSSES S. GRANT, 44

WTST VIROINIA, 12

MLL ROGERS, 49

WYOMMNG, 13

Gencent Election Corpthy

ANPD, Hetl Tinntior Reatator Frperiment

No. 1.45

No. 2, 45

$\mathrm{No.3}, 4$

Hote Rock Point Nuclear Piani,

Beowms Feny Nuckear Power Sintion Unh L, I

thin 2.

Whit 3 ,

Bownulck Stem Ikectrite Mapl

Unik I, Unik 2 .

C Reector d1

Gtin, Reputilo of, 21

Cinwon Power Sintion, Unit 1,

Cooper Nuclatar Sution, 2

DR Rpaptor, AI

Dentroyer Rouctor Pototypo, 45

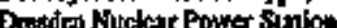

thinit 1,23

Unit 2,2

Unl: 3,2

Dunn Armald Bneray Center, Unit 1,2

Buth L. Halch Nuolear Plum

$$
\begin{aligned}
& \text { Ưpi 1, } 2 \\
& \text { Unil 2, } 2
\end{aligned}
$$

Engtincering Tasi Retctor, 33

Botios Petml Akomlo Row or Pland Unt 22

BSADA Yalledios Buporlogentel Supertot Renciot, 29

Genenil Elecurlo Nuctear Test Reactor, 7

Gempuny

Kalil Nuclear Powter Sution, 46

Kemkratwork-RWB-Bayennerk,

$$
\mathrm{KRHI}, 46
$$

Grend Git Nuctoes sinton, Unil 1,2

B Rejedor, 41

Howdi Nudear Pant

Unt 1, 2

Unin 2,2
Gep:onl Blectrio Compeny (Contined)

Hope Creck Nuskater fonteming sintion, Upit 1,2

Hombold! Bsy Powner Planx, Uni1 3, 2I

Inda, Tazapur Nuclear Power Siuten

$$
\text { Unit 1, } 15
$$

Inty

thin 2,16

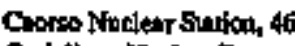

Gadgltano Stuclean Power Station, 46 Imes A. Fitepalrick Nuctear Popper Plant, Iopm

Fukuthina Dutisti Power Stution

Uhit 1, 16

Unit 216

Uhut 16

Jnpan Powt Demonarration Resctor, 46

Kethiwazaki-Karitm

Uhil 6, 18

Unit 7, 18

Tokai Na. 2 Power Sistion, 16

Tonuraga Power Sistion, Un't I, 16

KE Reaclor, 4]

KW Rescior, 41

La Salle Cowny Siction

Unit 1, 3

Unit 2,3

1olend Stanford Ualverdily, 39

Limerick Generuing Stalko

Undt t, 3

Unit 2,3

Mossachinsexi, Univertiky of, 9

Mexico, topun Verios Sinlon

Unit I, 16

Unik 2, 17

Miflesoma Nuekent Power Starion, Unti 1,3

ModIIlcations and Addillows to Readoc Farilits, 14

Montiostlo Nuclear Gearning Pinu, 3

Neiherimedic, Dodowand, 17

Nine Mile Poin Kutctese Suction Uail 1,3

Uall 2,3

Oyster Creak Nuctedr Powes Fiant, Inth 1,3

Peach Bumom Atomit Powror Sumion Ualt 2, 3

Uail 3, 3

Peny Thestoar Hower Elant

Uall 1, 4

thil 2 s

HARTFORD

HEL BNA, 12 
Genend Elextutic Compeny Cominues

Pignier Nocler Power Suliog, Uhk 1, 6 Qnendoviles Sintion

Unik 1,4

1hit 24

Rtver Bend Stallion, Until 1,4

Sharthm Notloar Ponver Sintion, 28

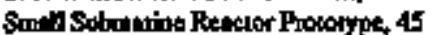

Sondhwert Bxporinental Part Cxidto Reacter. 31

Spin

Cofienke, Unik I, IT

Nucker Baed Boation, 20

Sinti Maria de Gurone, 17

Yoldecabaliores

Utit 1, 18

unt 2,18

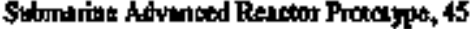

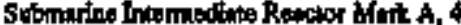

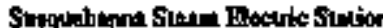

Unh 1, 5

Unings

Sirtzondand

Leibsuds, IT

Timb

fulitaber: 17

Chinukan

Uhia 1, 17

Uni 12,17

Krosteng

Unh 1, 17?

Unit2, 17

Thdem Prolotype, 14

Valberiton Boiling Waler Reactor, 31

Ventrouthe, 21

Vamoont Yarlike Noclest Power Sution, 5

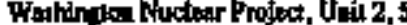

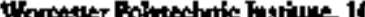

Generil Electro Nhdear Tart Reaclor, NTR.?

Oentelal Electric Teal'g Reactor, GKTR, 33

Genenat Nodear Bnpineering Corp.

Fionith, Uaviverity of 8

Atomt it Favitinle of Tecinolosy, 8

Genewa, Utiventily of, Swilzedind, 47

GEOROE BANCROFT, SSBN6 3, 44

OBOACB C.MARSHALI, SSBNW54,44

GEORGE WASHIHNGION (2 Tatton) I3

GEORCB WASHINGTON, SSN598, 49

CDORG WASHTITTON CARYRR.

5 Shanso 44

CSORGIA, SSHN729, 12
Georlis Intithe of Texhnology ACN-201-104,39

GIRR, :

Georala Powwer Co.

Alven W. Yogike Nocilear Plank, Unit 1.1 Uth 2 I

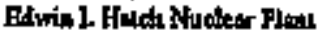
Unit I, 2

Grintany Whin 2,2

Asocilution for Radtation Rerrarth XR-OPS, 47

Anown Boverilivulep, XR-062, 47

Dinnstadl, Utilwetiky of, XR-009, 47

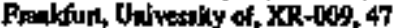

ERG-1, XR A08, 47

ERM Goweting, XR-004, 21

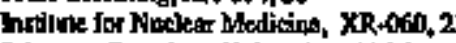

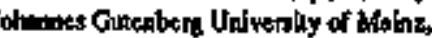
XR 0 OSO, 21

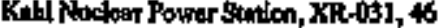

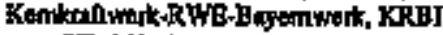
Xp.06\%, 46

Sedical College of Fanover, XR-0X, 2

Molhastan-Kretteri, XR-11s, 15

Minitch, Technfeti Untivertiky of, 21

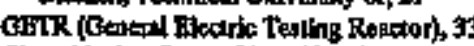

Ginna Nuctear Power Pint, Uah t, 4

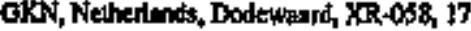

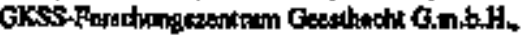

Gomang, FRO-1, XR-008, 4

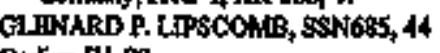

Codivn.F, 22

GPU Nirdeor Corp.

Oynter Creek Nockent Rosrer Planh, Otrit 1, 3

Thres Mile Islend Fitclaar Sytion

Unt 1,5

Unt 2,2

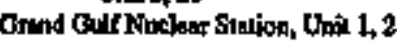

GRAYLDVE, SNN646, 11

Oreal Bricin, S5W for HMS DREADNOUGHT, S5W, 47

Gnece, Democillos, XR-GH4, 20

ORBENIDFG, SSNG14, 44

GRFENVI LE, SNFT2, 13

GROTON, SSHE94, 11

Ground Bxpedinemal Fingine Experiment

XE-Eackin, 32

XE-Prine, 32
Ground Teal Reactor, GIth, 46

GTRR (Goorgin Torh. Rusasen Reador). 8

GSARDFEH, SSNGI2, 43

GUTARRO, SSNG6, 14

GURWARD, SSNG62, 44

HRerctor, Rimhnd, 41

Hoddan Nook Plan1, 2

HADDO, SSNGOA, 43

HADDOCK, SSF621, 44

HALXBUT, SSN587, 43

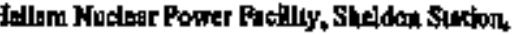
27

HAMMERHEAD, SSNG63, 44

HAMPTON, SSNTST, 13

Hentond 305 Tox Reator, HITR, 12

Hextin Nhucloar Powex Plant, Dalk 1,4

Harty Diamond Labomitariox, USA, Ditanond

Ondunes Rediulon Fucity, A6

HLARRY S. TRLMAAN, CVN75, 14

HARTFORD, SSN768, I3

HAWXRIL, SSNG6, 11

H.B. Robinion Plunk, Und1 2, 2

Horl't Phyjics Rusench Rearcor, HPRR, 37

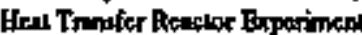

No. $1_{+} 45$

Na. 2,45

$\mathrm{Na} 3,45$

Beavy Worer Componends 'Teit Reictor,

HWCTR, 30

GEE ENA, SSTMS, 12

BENRY CLAY, SSBN6Z, 44

HENRY L. STIBHSON, SSON655, 14

HENEY H, JACKSON SSENt30,12

APER (H)ten Finx Bearo Roactor)?

EFIR (Aligh Fhe Inotope Reacolor), ?

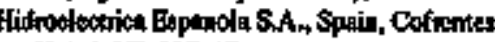

$$
\text { Unit 1, XR-097, } 17
$$

Figh Faux Beant Rotockor, HapBR, 7

Hitgh: Finx Jeotope Roantor, tifR, 7

Fight Tempopingra Iatice Tan Reactor, HJLTR, 37

Hombigneous Reschor Frperimanl

Na. 1, HRE $+1,30$

No, 2, HRE-2, 30

Ktonsyecrnb, 2

HOHOLULd, SSN718, 12

thops Ceeck Nockear Genscillug Station, Uhit 1, 2
Hadrome1, 48

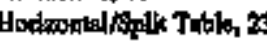

HOISTON, SSN713, 12

Honslon Llshalng \& Powor Ca, Sount Texa

Projeot

Unil 1.4

$t_{n+12,4}$

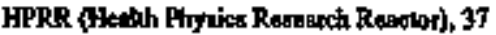

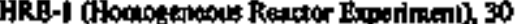

HRE-2 (Homotomeons Rauctor Exporimeni), so

HTL,TR (High Tenperalupe Labtoc 'TeH Retoctar. 37

HIR (thuford Test Reactord \$2

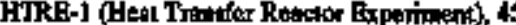

HTRE-2 (Hetl Tramiler Regctor Expetinenk), 45

HTRE-3 (Hast Trunfar Reactor Exporimeni), is

HTTF (Bellir), 48

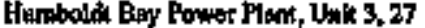

HWCTR (Henvy Wake Componends Toat Resctor), 30

HYMAN G. RICKOVER, SSN709, I2

HYPO (Los Alimos Waver Hoiks), 37

Idabo Nwoker Corporatiost, Spectal Powrer

Bxerarion Retclos Tast No. 4, 35

Idabo Sinte Llaiweraly, AON-201P-103, B

Illbots, Undversiby of

LOPRA, :

TRUOA-N4K II, 8

Illinois Intitnte of Tocbrodosy Rosaurch

Indiluk, ARR (L-S4), I7)

Illmota Rower Ca, Clnton Pawer Sution, Unil 1 ,

Inperial Chepderl bidoentries, Englond, XR-074,

Inden, Tanapor thuodear Poprer suction

Unit 1, XR-054, 15

Unit 2, XR-054, 16

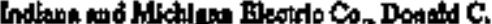
Cock Nuclear Power Plont

Unil 1,2

Unil 2, 2

TNDANAFOUS, SSTNG97, 12

bodiun Poim Sution

Uaik 1,27

Unir 2,2

Jaik 3.3 
Inderation, Notional Avortio Enetoy Atenct Bundeng 20

Yogfotictits, 20

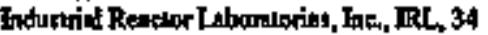

Ingelle Shipboildng Cor

ASPRO, 44

BARB, 43

$\mathrm{DACB}, 43$

HADDOCK, 4

PARCHB, 11

PUFFR, 4

SCIULPN, 49

SNDOK, 13

TAUTOG, 1$]$

TUNDY, 11

HIJLAM H. BATES, 11

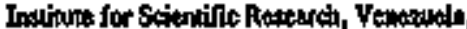
XR-018,21

Intibut of Fucleat Afrins, Colombla, IANRI, XR-05s, 19

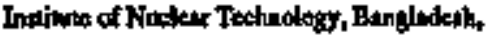
XR-125, 19

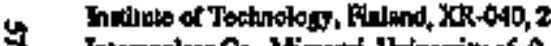

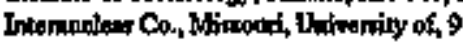

Iowa Electic Lijo \& Potite Ca, Ditate Arrold Inersy Cenuex, Unil I, 2

Jowa Stav Lriveriky, UtrR-10,

In, Uawenly of Tehrat, XR+029, 2 !

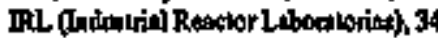

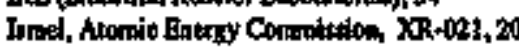

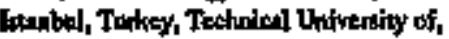
XR-108, 21

inty

Cono Norlaur Station, XR+OMT, 46

Cemine for anllary Application of Nuclen Energy, XR-016, 4?

Europen Commbrily Conurioston, XR-007.

Fin TT, XR-016 of

Gadglines Shdear Powar Simtion, XR-043, 45

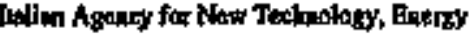
and the Envinominenl,

Cunocin, XR-1051,47 Roune, XR-126, 20

Milin, Untwenity of, XR-42, $\$ 7$ Psitimo, Univetiky of, XR-025, 22

Pavin, Ualirertily of, XR-056, 22

Trino Vereellese, XR-044, 45
IACR, Ssitoss, 43

JACKSONYILLE, S\$N 599,12

JAERI, Jngun, Jypen Power Dementuntion Resctor, XR-1045, 46

Jmitich, Kingtoos, XR-094, 20

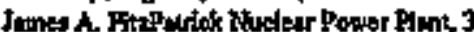

JAMES $\mathrm{X}$ POLX, SSIt6AS, II

JAMES MADISON, SSEN627, 44

JAMES MONROR, SSEN622, 44

JANUS Reacior, 3!

Japm

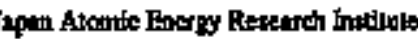

Itapan Poprer Depnoantedion Reactor, 46 JRR-2, XR-015, 19 NSRR, XR-101, 19

Toket Afum, Ibarnki Pref. XX8001,47 $X R-045,46$

Iaped Axortite Pownor Co

Toket Na. 2 Powor Simion, XR-OAS, 16

Tuumga Power Stoniog, Unil 1, XR-065. 16

Kapded Electric Powrer Co

Litame Powrer S1wion, Ualt I, XR-067. 16

Oht Powar Station

Itul 1, XR-1082, 16 Uhtik 2, XR-0.02, 15

Tikshame Power Stalion, Indil 1 , $\mathrm{XR}+079,16$

Xtakd Untivaniny, XR-041, 22

Mutuliti Conleze of Technolosy, XR-03T, 20

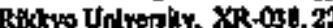

Tokno Ellotric Fomer Co.

Flaknglime Dri-ichi Power Stution Ut: 1, XR-065, 16 Jak 2, XR2,072, 16 Unik 6, XR-094, 16

Kechlungret-Eonten

tulk 6, 18

Uhat, 18

JEFEHRSON CITY, SSYYt59, Is

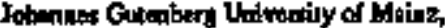

Gecon Iny, XR-050 21

JOHN ADAMS, SSBN 620,4

JOHN C. CALHOUN, SSBHW630, 44

JOHN C. STENNDS, CVN74, 13

JOHN MAAESHAL, SSN6II,

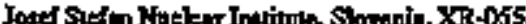

Josth id Furky Nockour Pax Unis 1,3

Lhit 2,3

Jugetmmi, 35

K Reackor, Swannah Rtwar, 10

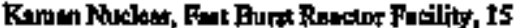

KAMERAMEHA, SSN642, t1

Kanatis Bectrio Power Co, Japen

Mihatine Powar Statinn, Innit 1, XR-057, 16

Chi Powt Simion

Uaik 1, XR-082, 16

Uthit 2 XR-0A2, 16

Thloheme Power Station, Unin 1, XR-079, 16

Kindis, Univertity of, Model 41:0, 39

Kanow Stals Unlwomity, TRTta hik D, \$

KE Retector, Breinend, 41

UCKY, SSBNT37,

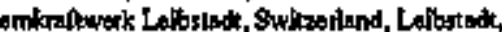
XR-104, I7

Kawannes Nucloar Power Mlank, 3

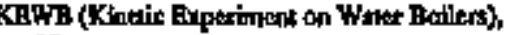
37

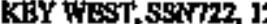

Krnetic Expetsueal on Wutor Boilent, KEBWB, 37

Khing intetest Newron Gentrmos, Kinglet, 34

Kingloh, 34

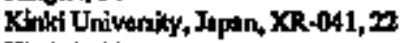

Kindah

Kiwi-A Prione, 39

KHWil-k3, 33

Xiwi-B1A, 33

XWw-Bits, 93

KWWITA4, 33

KivitgdD, 33

Kiw1-945, 33

Kiwt-Trantient Teat Reactor, KSwi-TTR, 34

Kiw-TTR, 34

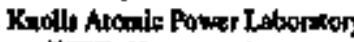

$\mathrm{ECFE}_{4} 48$

PPR, 48

PaR, 48

Themal Tet Reactor ito. 1, 46

Kore:

Atruneed Energy Reserech Inalumie

XR-027, 20

XR-ois, 20
Kopat (Comtimed)

Korea Eledric Porror Co.

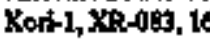

Xori-2, XR-119, 16

Rori-3, XR-131, 16

Kort4, XR-131, 16

Jlchin 3, XR -159, 18

ujctin $4, X_{2}-153,18$

Yonsfwang -1, XR-133, 16

Yonsgumeng, XR-193, 16

Yor 19 , wats -3, XR-15D, 18

Yongtwang-4, XR-150, 18

Yonygwang $5, \times R-167,18$

Yonugwang 6, XR-162, 18

KUKL,A, 38

KW Rowerer Richland, 41

Kyung Hea, Uniweoly of, Koren, XR-105, 22

L Renctoc, Alket, il

L-47

Atomites Helectentionu, 35

Brigham Yourg Un'wettky, 39

Caliomin, Sapic Britan, Univeratsy of, 39

Nevadn, Universily of, 40

Promio Rico Neolent Cealer, 40

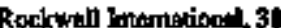

Wromtis, Unlverity of, 41

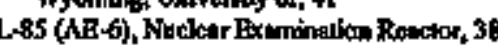

L. MENDELL RTYERS, SSN686, 12

Lo Crope (Genon) Nhedgur Geverating Siallon.

LFAYETTE, SSBN6:6, 4

LA JOLLA, SSANOL, I2

CAMPRE-I AOs Alamos Nokes Photiontum Rescior Eaperimen'), 30

LAPON, SSN661, 44

LAPRA+1 (Los Mindos Power Renctor

Experiment, 30

LAPRH-2 (Los Alumod Power Roactor

Exparimant), 31

Large Shtp Rusctor Polotype (2 reactors), AlW.

La \$̦Do Counly Stotion

Unith 1,3

Until 2,3

Lentice Teat Reaclor, LTR, 4

Lawrence L fivemore Notional Laborwory

Experimenicl Propution Tart Retetor TORY IIA, 45 TORY IC, 45 


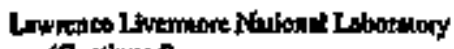
(Continued)

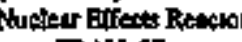

FRAN, 37

KUKL 1,38

Super XUKLL 46

1C) and 5 Sinford Utwenthy 39

LEWIS AND CIARK, SSAN644, 44

Imentik Gereraling Stalio

Uwin 1,3

Init 2 ,

LrrR (Low Invenity Ton Reactor), 97

livemore Pool Typs Resoter, LFTR, 37

1 livemore Woer Boiker, IIWB, 37

LITH (Livennore Wator Bo:1erh, 37

Lockheod Airch fi Com

Colombila, LN-RJ, ig

Cintod Fecllyy tor RFR, 4.

DOR Demoritintiton Remetos, 36

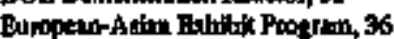

Lockheed Airmifi Cop, 37

SASA Hock-11, Reador, 3 ?

Ohio Sinte Unfuettity,

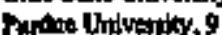

Ratitation Effocti Reactor, 38

Lockhosd Idilo Teshnologita Conpany Advanced Reacivity Neatilitial Facitity, 7 Mvanced Tear Retesor, 6

Cospled Pat Rewetinily Manturemeal

$$
\text { Facilits, } 7
$$

10FT (ios of Fhaid Tush 34

LONO BBACH (2 reacton), CON 49

lang Island Ligheing $C_{0}$, Shoretum Pfucker Powat Souben, 28

LOPO, 37

LOPRA (Low Power Reacior Astembity)

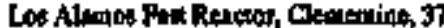

Los A tarest LOPO Reart ox, LOFO, 37

Los Alantor Wollen Mintonitun Reuctor

ExpesianenL, LANPPRE-1, 30

Los Alamos itulional labocilory

Bis Ten, 22

Cowlox 22

Flallop, 22

Godiw-ST, 22

Honasoomb, 22

Marn, 22

Pukn, 4B

Pinct, 22

SHFBA, 27

SKUA, 22

Vonus, 48
Lor Alanios Power Reactor Buporimenl

No, 1,30

Na. 2,31

Los Almos Wake Bollor

HYPO, 3T

SUPO, 37

LOS AMCELES, SSN6838, I

Lost of Fald Ter, LOFT, 34

LOUISAANA, SSBNO43, 14

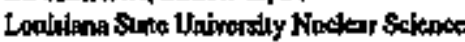
Center, SNARE, \$7

LOUSVILE, SSW724, 12

Low Intality Tent Rencior, IItR, 3 ?

Low Tesuperalure Nentoot Imodimiton Fudility. 1,TNIP, 37

1.PTR (Livesnore Pool TYpe Rencter), 37

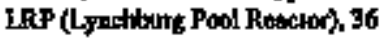

LTR (Litioe Tet Rewarory, $\$ 2$

LWBCC, 48

Lynchborg Reseanch Gonlen, 48

MANA, SSBN741, 13

Maine Yonlese Atortit Powsr Pmonl, 3

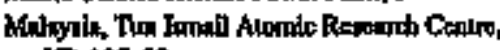
$X R-125,20$

Mmhilin Colless, MCXRR,

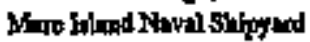

ANDRES JACKSON, 4

DANIEL BOONE, 44

DRUW, 44

OUTTARRO, 44

GURWARD, 44

HALABUT, 43

HAWXBחJ, 11

KAM텨 AMBDitA, I

MARIANOO O, VALLEOO, 4

PBRATr, 49

PINTADO, Is

PLUNGER, \&\$

SARGO, 43

SCAMP, 43

STONBWWALI_JACKSON, 4

THEODORB ROOSEVELT, 43

WOODROW WT SON, 14

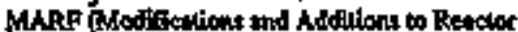
Patulityly, 14

MARLAND G. VALLEO, SSEN658, 44

Man, 28
Martin Mordesin Corp.

Pontule Motian Power Ptum No, 1, 42$$
\text { No. } 9 \text { A. } 42
$$

STUROIS Plowing Nuclear Power Phan, 43

MARYLAND, 35ANO36, I2

Maryland, Ualwoanhy of, TRIGA, 9

Mantucheaseds, Upiveryily of, UIR, $\$$

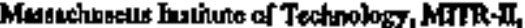
9

Matarials Teting Resetor, MTR, 34

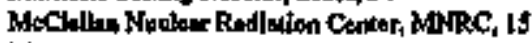

MeOinire Nudsar Staliba

Uait 1,5

Unit 2,5

MaMtorler Unlwershy, Conado, XR-011, 21

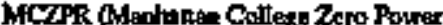

Rowctor), 9

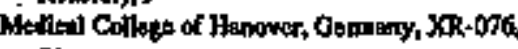
21

MEMPFIS, SSNOD1, 11

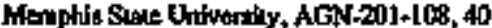

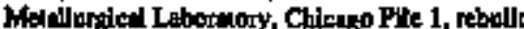
af 6 , 2, 36

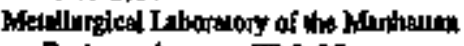
Enginser, Argouno CP-3,35 Mexiso

Loginat Vado Stallon Uait 1, XR-098, 16 Uait 2 XR-10R ]?

Notional Comanlation bor Nockar Bnersy. XR-057, 20

Silazar, 21

Mrt-1A, 43

MIAMI, SSFTSS, 13

MKCHUAN, SsENm, 12

Michigan, Uniwetrily of (Ford Nuclear Reactar), FNR,9

Mlediem Stave Untvertily, TRIGA-Mik I 40

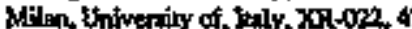

Millenoce Nudtear Power Stution

Unit 1, 3

Unt 2, 3

Until 3,3

Minat Otemla, Uhiwersixy of, Boril, XR-028, 21

MDNEAPOLIS-SADVT PACL, S\$N708, 12

MISSISSIPPI (2 reactors), DON 40,13

Mërintip pi Sate Universily, RRR, 40

Matururi, Untwority of, MURR, 9
Hhowit at Rola, Univerity of, UMR-R,9

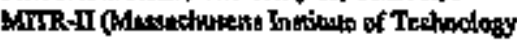
Reastark 9

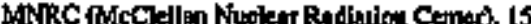

Mothlo Low Porwer PIun No. L, ML-1, 45

Model 4130, 39

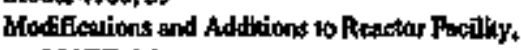
MARF, Jd

Molven Sall Renctor Erperingat, MSRE, 31

Mentoedlo Nuckent Oeneraing Plenl, 3

MONTPELIFR, SSN765, is

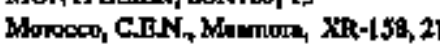

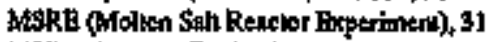

ATR (Materisls Tonting Rescter), 34

Hhrich, Gomany, Todnikat thiveratly of, XR-004, 21

MUR (NASA Mock-Up Roctor h 37

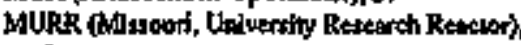
9

Mostadi Colloga of Technolosy, Japen, XR-037. 22

NRtiaton, 28

NARWHAL4 SSNGTH, 1]

NASA Mock-Up Reactor, MUR, 31

NASh-TR, 34

NATEANAEL OREENE, SSBN636, 44

NATHAN HALLE SSBWH23, 44

Kedional Aonopapliss and Spece Administration.

Pron Brook Rosctor Parillity, 34

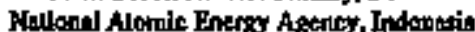

Bindung 20

Yogyakmea, 20

Nutional Commistion for Nockar Entroy, Mrrico, XR-0ST, 20

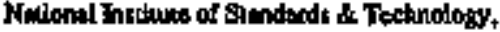
NBST, 7

Netional Laboralory of Fnginesicrs and

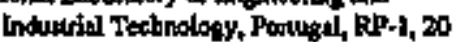
Nedionel Power Corp, Philippirica, Reputitic of

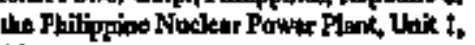
18

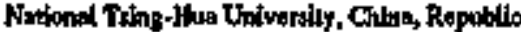
of, XR-620, 21

Ninuni Circulation Teat Phom, sso, 45

NA1FTILUS, SSN57t, 43

Novd Reyewith Rorclot, NRR, 46

NHBRASK A,SSBPTS9, 12

Nebyanka Probtle Powar Digtrict, Cooper Hioclear Sulion, 2 
Nowheringion

Delfi Testried Univerity, XR-0,03, 2 Dodewand, XR-D58, 17

Eneryy Cerive, XR-013, 19

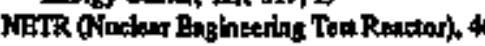

Newnos Rutiogniphy Pucitis:

NRAD, 7

$$
\text { NRF, } 37
$$

NEVADA, SSBNT3, 12

Novedn, Univanity of, L 77 , 40

Bur Merica, Univenity of, AGN-2011M-1 12, 9

Ketport News Shipboilding \& Dry Dock Co.

ABRAHAM IINCOLN (2 rescors), 13

AtBANY, 13

ARKAWSAS (2 tetuors), IS

ASHEVLLE, IS

ATLANTH, 12

BATON ROUtGe, 44

BERMIIFCHAM, 12

BOISE, 13

BUEPALO, 12

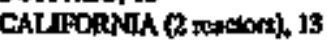

CARL YINSON (2 resctosi), ls

CHARIOTTE, 13

Y) CABYYINNE, 13

CHICAGO, 12

CINCMNATH, 45

DWIGIT D. ELSBWHOWFR (nactors), 13

DRTFRFRISB (8 tecion), 3

FNBACK, 11

GEOROB C MARSHALI, 44

GEOROE WASHINGTON (2 reston), 13

GEORGE WASFIINGTON CARVER, 4

GREFNYILCE, 13

HAMLERHBAD, 4

HAMAPTON 13

HARRY Y. TRUUAAN, 14

HERTY CLAY,A4

MONOLOLU, 12

HOUSTON, 12

JAMES MADLSON, 44

JANES MONROE, i4

JEPF FiRSON CITY, 13

OAW C. CALFOUN, 4

MOHNC. STENDLS, 13

JOAN MARSBAIL,

EEY WEST, 12

LAPON, 4

LEWTS AND CLARK, 4

L. MENDELL NTFER, 11

LOS ANGEL IBS, 11
Newpon How Stipbailding \& Dry Dook Co.

(Conlinuet)

MEHPUIS, $\mathrm{t1}$

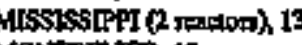

MONTPES ER, is

NEWEOHT NBWS, 13

NEWPORT KBW', SSMTO, 13

NTMTIZ (2 resctors), ts

NORFOLX, 12

OKLAHOMA CTIY, 12

OLYMIJA, 12

QUERTAFSL, 4A

RAY, 44

RICHAARD B. RUSSELI

ROBERT H. LEH, 43

RONALO RBAGAN, 14

SAIT LAKE CITY, 12

SAM HOUSTON, 13

SAMBAYEUIN, 11

SAY FEANCTSCO, 12

SCRANTON, 13

SHA DEVL, 44

SHARK, is

STHON BOLYYAR, 4

SOUTH CAROLINA (2 Detotots), I3

SPAOBFSth, 1]

THXAS (2 Exectota), 45

THEODORB ROOSEVELT (2 ructors), 13

THOMAS JBFFERSON, 44

TOLEDO, I3

Tocson, 13

VIRCIINIA (2 mation), 45

VON STEXIBAN, 44

NE" YORK CITY, SSNE\%, 45

Nisw Yoik Portot Authority

Indtan Roint Stalion, Unil 3,3

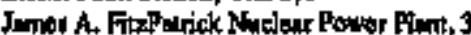

Now Yonk Shipbotildiag Corps

CUARDEISH, 43

HADDO, 43

Nudour Ship SAYANDAH, 29

POLLACK, 43

TRUXTUN (2 neactor:) 4

POQY, 11

Angean Bhownk Power Coos

Nine Mlo Point Nuclear Sution

$$
\text { Unte 1, } 3
$$

1 it?

NIMITZ (2 newcot), CVN68, 13
Mine Ailt Poin KhelearSinton

Uhit 1,3

Unition

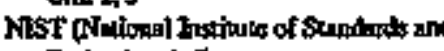
Techeolowy) 7

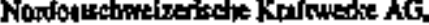

Swlizinand, Berouap

$$
\text { Lish 1, XR-063, } 17
$$

thit 2 XR 0170,17

MORFOLK, SSN714, 12

North American Rockiwell, Nuster Eranination Rencis, LA65 (AE-6), 38

Nonl Amat Power Sirtion

Unil 1, 3

$$
\text { Unils 2,3 }
$$

Norik Aluoto Enery Servico Corp., Sombroot

Nuclear Stution, Unt 1, 4

Nonth Canotina Stats Univtrsily

Graphic/wriker, 40

PUISTAR, 9

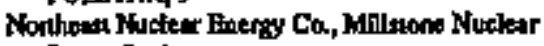
Pow Station

Unit 1,3

Unit 2,3

thitt 39

Northem Siletes Rowarco.

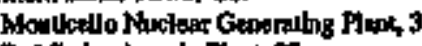

Pnlfindser A10otic Plank, 27

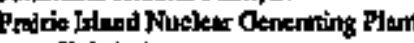

Unik $t_{4} 4$

Unir 24

Nothrop Corponat Lebontories, TRIOA A-Mk F, 37

NRAD (Nen'ron Ratiogndry Facility, INEL) ? NRF (Neworon Rediograply Fuciky, Hotlotd).

NFR QNimal Rerearch Renctor), 46

NRX-A2 (Noviear Rocket Engine Ropenot, 32

NRX-A3 (Noolear Rocket Enoine Reaclor), 38

NRX-A4,EST (Noclear Rocker Bngine Reactor) 33

NRX-A5 Ructear Rocked Fingin Reacior) 52

RRX-A6 Ohudear Rockol Burior Ro:acloc), 32

MTR Oincleur Teal Reaclor), 7

Nurdear Bffect Reacior

FRAN,37

KUXLA, 38

Sopor KUKLA, 46

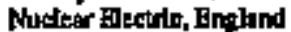

strewn: B, XR-148, 15

Mudtar Entery Bowed-JEN, Spain, XR-010, 20

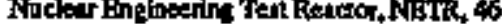

Nuclear Bxumination Rerctor, 1-BS (AR-6),

Nuckear Meditine, In tilute tor, Oemeng, XRto, 0,21

Nuckes Rekench, inatume for, Romein TRUGA-ACPR, 19

THOAA (MPR 16), 19

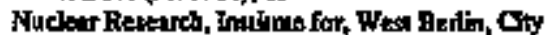
of, XR-012, 4

Noder Rocket Engine Retstor Bxpsingox QNERVA

$$
\begin{aligned}
& N R X-k 2,32 \\
& N R X-k 3,32 \\
& N R X-k 5,32 \\
& N R X-k 6,32
\end{aligned}
$$

Nincluar Rockel Reudor Engino Sysien Tell (NERVA), NRX-A4EST, 33

Noclear Rocket Reiclot Frpetiunow'

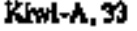

Kiwl-A Pinime, 33

KJwi-A3, 33

Klwi-BIA, צy

Kivi-BIB, 33

Kisi-B4A, 33

KJwt-B4D, 33

Niwi-14E, 33

Phosbur 1h, 35

Phoobuo 1B. 3

Phocbun 2A, 33

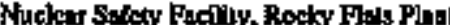

Horizonleatspiti Tabio, 23

Solution Buse, 28

Verlcolispill Table, 48

Waler Reflectar Tmok, 48

Nucketr Stip SAVANTAH, 29

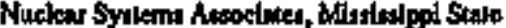
4 niwerdty, 40

Nuklenme Ellchapme Rrdko, Skownin, Rrsko, XR-107, 17

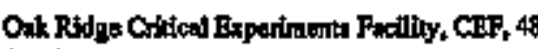
Onk Ridge Gntphilt Retotr, ORO, 38

Oak Radige Naxlonal is lborstory

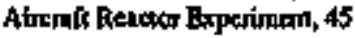
Buly Shidding Retector, 36 IFOB Denanulntion Renclax, 36 Heglin Physics Rexcmch Reactor, 37

High Flux Itrotopo Rescloc?

Homeseneoul Beachar Bxperimenu No. 1, 30 
Oak Rididge Nutianel Lebontiogy (Condrued)

Homosonecau Revetor Brpetimew No. 2, 30

Low Intenulty Test Reacter, 37

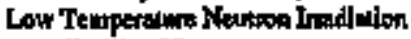

$$
\text { Fan'tity, 37 }
$$

Atolien Salt Reactor Experinal, 31

Oak Rilito Renemdi Reictor, 34

SNAP-02jOA TSFShichding Experiment, 31

Stribktend, 20

Tower Shlialditis Rewast

TSR, 29

TSR-2, No. II, 3:

Oat Rid gu Rereonch Reacior, ORR, 34

Oxones ituctorar Stulon

Ualt 1, 3

Unit 2,3

Uat 3, 3

Ofice of Alomic Bergy for Peace, Thailand.

TRR-1, XR-112,21

OrFo, SSBA7 26,12

Obia Sun Un'vethis, OSURA, 9

Olalintone, Upivertixy of, ACN-21 1-102, 40

OREAHOMA CITY, SSNT23, 12

OLYMFIA, SSNFI7, 12

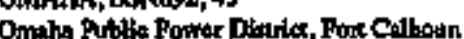
Stetos, Uhit 1,2

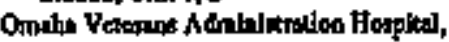
TRICA-MIKI, ?

Onemp Wost Reaciar, OWR, 34

OMRB (Orgenk Moderiad Reantor Experipent) 31

Oregoes State 1hutwerithy

AON-201-114, 40

OSTR, 9

Orgunic Moderuled Resctor Foppetiment, OMRE, 31

ORG (Oat Ridise Ginthe Reactoc), 38

ORNL Pool Cidiod Astenbly, BSP Poot PCA, 4I

ORR (Oak Ridos Reseurch Reuctor), 3 CSTR (Ortoon SAre Univeratly Restcon) 9 OSURR (Ohio Sinl: Letwansily Reverech Rentitot), 9

OWV (Omena Wost Reacert, 34

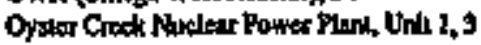

PReadior, Aiven, 41

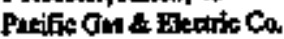

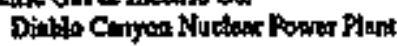

Unit 1,2

Uhik 2,2 .
Puntio Powrer Gin a Elearic Co. (Continned) Hurbaitk Boy Power Plont, Jan 3, 27 Vullesilos Bodling Wons Reretor, VBWR, 31

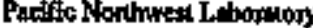

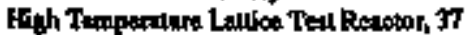

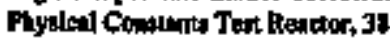

Themen1 Ten Reactor No, 2, 38

Pakizan, PARR, XR-046, 20

Pilesno, Unwerdiy of, Ituty, XR-025, 22

Pelintda Noctear IJand, Unta L, 3

Palo Verdo Neclear Gemeralint Suntion.

Units 1, 3

Uhit 2,3

Unis 3, 3

PARCAHE, SSN601, 11

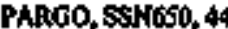

Puth 48

ASADENA, SSN752, 13

Puhfindor Alonitc Phat, 27

PATRICK HERTRY, SBN599, 43

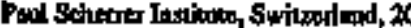

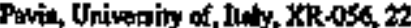

Powing Restarch Retactor, PRR, 36

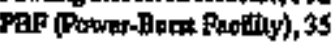

PCA (Pool Cotikal Atstmily), 48

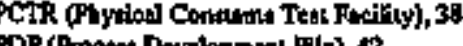

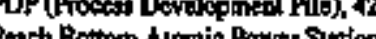

Lhit 1,27

Ditr 2,3

Uinest 3,3

Penn Stale TRIOA Retelor, PSTR,

PENNSYL,VANIA, SSPNT35, 12

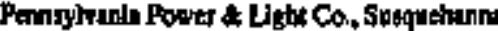

Sugan Bloordo Sumtion

Utuit 1,5

Unt 2,5

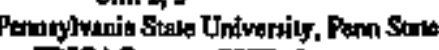

TRHA Reaclor, PSTR 9

PHRLLT, SSNS94, \$3

Penty Mucloar Power Plan:

Unit 1, 4

Unit 2,5

Pewet-1,32

Pewte-2, 32

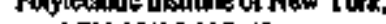

Fhiladebpia Blactric Co.

Linterkt Oneraling Stwlon

Utill 1,3 .

Unil 2,3
Fhllwdelphin सhestric Co. (Conlinoed)

exch Bottom Aluondo Powrer Sution Unit 1,27

Unil 2,3

reipining

$$
\text { Unil 3,3 }
$$

Eepratice of dh, PRR-1, XR-034, 20

Reputing of the Phillippines Nuclear Pow Pland, lint $1, X R-120,18$

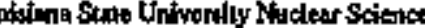
Center, SNARB, 37

SNAP-104 Thasticnt Tat No, 2, 35

Special Power Buenrition Reator Teat No. 1,35

Non 2, 35

Prowber

IA, 33

IA, 39

PHOEND, SSKOTO2 L

PAREINIC, 36

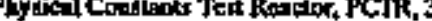

PIFTAD Nockatr Power

Piquin Noclear Powror Facilliy, 28

FTTSBURCH, SsFro, 12

Plannt, 22

Whm Brock Reactor Faclliky, NASA-IR, 34

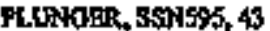

Pirtenium Recyck Text Rewckr, PRTR, 31

PM-1, 42

PU-2A, 42

PAL-3h, 12

FNLC Critical Mast Laboestocy

FEAS, 48

Horizomial, 48

RSTM, 48

POOYY, SST647, II

Point Heach Nuclesr Flin

Uak I, 4

Unit 24

POLLACK, SSNGSO3, 4

ACN-20134-105, 40

Portable Mediont Power Pient

No. I, PM+1, 42

No. $2 A, P B 2 A, 42$

Wa. 3A, PMSA, क

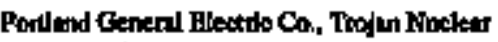
Pint, Utwil 1,28

PORTSMDUTH, SSM707, 12

Porlsonouth Novil Stipgad

GRAYLAVO, !1

JACK, 43

JOFIN ADAMS, 44

NATHANABL GRHENH, 44

SAND LANCE, 11

SRADEAGON, 13

SWORDFISH, 49

TTRRESHER, 43

TINOSA, 43

Portngal, RP+1, XR-013, 20

Pontr-Aurat Inoiliny, FBP, 35

Poprer Reacior Developopent Company, Earileo Penti Akmis Power Plex, Unil 1,27

Prairí Iotand Noctcar Genoraling Plmt

Inite 1, 4

Unit 24

Procean Devalopgemtex Pids, PDP, 42

Prajest BNEL of SENN, Kaly, Garigliano

Nucketr Power Siation, XR-143, 46

PROVIDIENCE, SSN7 I9, 12

PRR (Puwling Restegrch Rewclor), 38

FRTR (Finicnian Rocyato Toal Reaclory, 31

PSTR (Pent Stal TRIGA Fucility), 9

PTF (Prolf Test Facitily), AB

PJR (Pool Test Ro:ator), 48

Public Servica Co, of Colonado, Port SL Vais Noclear Genorming Stution, 27

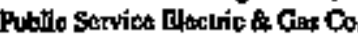

Hope Coekk Nuclear Gentating Stuion

$$
\text { Int 1, } 2
$$

Sulem Muclagr Genorating Stulon

Unit I, 4

Unit 2, 4

Puerto Rica Nuclear Center

L.T.40

TRIGA-FLIP, 40

Puerto Rico Walce Resourees Authorty, Bolling

Nocker Supenteder Powar Stetion, 27

PUEFR, SSNGS2, 44

PUL,STAR * Nonil Comolia Stose Uhtwertily, 9

Pundue Univentily, PUR-1,9

\section{Quad-Cinios Stalion}

Inik 1,4

Unin 2,4 


\section{REACTOR INDEX (ContInued)}

QUEENASH, SSN65I, 44

Qeen Bary College, London Uhưvenily, Uriled XIngdan, XR-04, 47

Quinct Divilion, Gevenl Dynuries Corp. GATO, 44

GRBENIING, 44

SUNFSH, 11

WHALL: 44

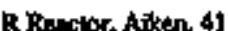

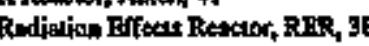

Renchos Stoo Nuclew Geserting Salion, Unil 1, 28

RAY, SSH657, 4

Reod Collasa, THIOA Al I, 9

Rogiond Ceniter for Noclear Studies, Z,ite, 21

Retussliver Polyzedulio Inalinute, Troy, NY, 22 RER (Redloulon EItocts Reackor), 30

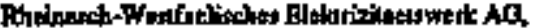
Gomastr, Mulluein-Kaefida, 15

Rhino-Wenthulie Parter Co. RWE, Osonany,

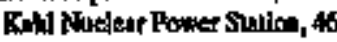

RHODB ISLAND, SSENT40, 13

Rhods Inland Nactear Science Cenine, RINSC, 3

Bice Un'ventily, AOTH-2]I-10L, 41

RICELARD B. RUSSBII, SSN687, 44

Ridkyo Uawedily. Japun, XR-09, 22

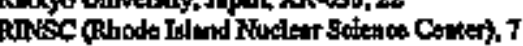

Rito Eniond Labomitory, Dennark

DR-1, XR-AN, 19

DR-2, XR-006, 4

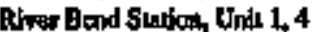

ROAFrT E t.5t, gSNo01, 4

Roben Enmer Ginin Siclear Power Flast, Unlt $I_{1} 4$

Robirston Pan, Unit 2, 2

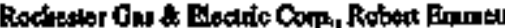

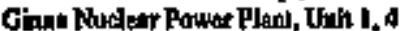

Rockwall Interinationt

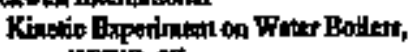
KSWB, 37

L77,3!

Ptrods Ifland Whelese Selences Cenler, 7

Rominin, Insthaxe for Nactour Reseanch

TRIG $\lambda$-ACPR, 19

TRECA (HIR 16), 19

RONALD REAOAN, CWN6, 14

FRR (Etaloigh Berearth Reacion), 40

RSTM, 4 .

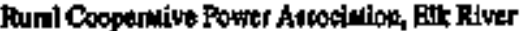
Beator, 27
S10RS-1, 32

S10FS-9, 32

$\$ 0 A-4,31$

S10F-5,32

919. 45

SIO. 45

$\$ 1 w, 45$

SiOS, 31

596,45

550,45

StDR, 31

SgER, 31

$\$ 00,14$

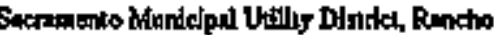

Scos Woctsor Generaling Sintion, Unis 1,29

S. Indo Plant

Inis 1, 4

Unil 2,4

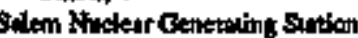

Unt 1,4

thin 2,4

SALT 1_AKE CTT, SSN716,12

SAM HOUSTON, Ssti609, 43

SAM RAYBURN, GT-SSENOCS5, 11

Sindia Eatineoring Reator, SBR, 39

Sendia Netionll liboctuodet

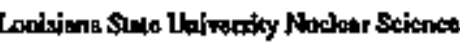
Cenler, $3 T$

Sandis Fngineteting Reuctor, 3 A

Sandite Poled Recilor, 38

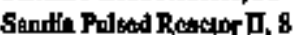

Stndir Pollopd Resctor II, 8

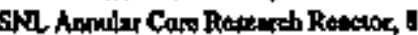

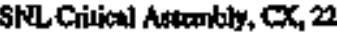

SANDLANCE, SSI660, :

SAN ERWNCTSCO, SSN711, 12

SANJUAN, SSFT51, 13

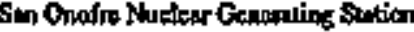

Ihit 1,28

1hik 2,4

Uall 3,4

SANTA PB, SSN760, 13

Sw Ranto, Untwerdly of, Bnwi, XR-002, 21

SARGO, SSN 583,43

SAYANNAH Nucker Sh'p, 29

Saton Studear Itsperimenial Corp, 31

SCANP, SSN5:99, 43

scitro, 16
SCCFPION, SSN580,43

SCRANTON, S5STH6, Is

SCUL.PN, SSALS\%, 43

Seabrook itudeur Station, Unik 1, 4

SBA DEVL, SSN664, 4

SBADRACON, SSN564, 43

SEA HORSR, SSIN609, 44

SEAWOLF, SSN2I, 19

SEAWOLF PWR, SSNST5, 43

SEA WOLF Sodiun Reactor, SSNSTS, 43

FFOR (Southout Brpedmequal Fuss Ozito

Reaclor), 31

Sentar, Aunrib, Asura

XR-023, 19

Serrominh Nutdar Plin

Un'l 1,4

Unta 2,4

SER (Stundia Eigincering Retctor), 34

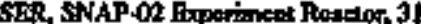

SF[ARK, SSWSG], 43

Shearos Harrio Nuclear Power Pland, thk 1, 4

SHEBBA (Solntion high wangt bard nopembly)

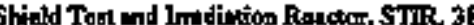

Shypondopon Atornic Powor Staton, 28

Showeha on Ficleat Power Station, 28

SILVEREDES, SSNoth, 4

SIMON BOLTVAR, SSBNIGA1, 4

SKATT, SSNS78, 43

SKIPJACK, SSNS5BS, 43

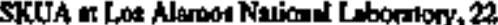

SI-L (Simioany Low Power Pini), 45

Stowenim

Joter Steinn Noclear Intrillode, XR-055, 20 Kriko, XR-19, 17

SM-l (Stalionary Modinm Power Pien), 43

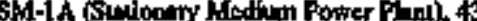

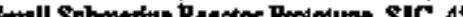

STAP-C2 Dovalopmenial Syutem, S2DS, 51

SNAP-Q2 Bxperintall Reactor, SER, 31

SNAP-02HOA 'STP Shieldirg Expentment, SNAP-TSF, 31

SNAP-Q. Devekoment:1 ReBclor, S8DR, 31

SNAP-0S Baperinenill Restor, S6ER, 3t

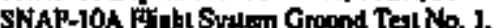
\$10Fs-1, 32

SNAP+10A Fitght Syam Ground Teatio. 3. \$10\%s, 3,32

sNAP-10A Fight Syuten, S10Fs-4, 3

SNAP-10A Fingt Sylem, SJOPS-5, 32
SNAP-10A Thatient Tes No. 2 SNANTRANA2,35

SNAP-10A Thustiem Ten No.3, SNA PTRANA3,35

SNAPTRAN-2, 35

SNAPITAN-S,35

SNAP-TSF, 31

SNARE, 37

SNL Anndur Core Reasarui Reactor, ACPR, B

SNL C.piced Aarwably, CX, 22

STFOOK, SSN 592,43

Soditam Ratctor Brpasinent, SRB,31

Solotion Buts, 23

Souch Airites, Safand 1 , XR-042, 19

SOUIH CAROIJNA (2 tenctoti), CCLY7, I3

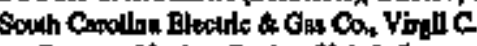
Surmmer Nocleur Siulan, tin't 1,5

Savh Teras Projed

Uali I, 4

thin 24

Southem Cabronia Eatson \& Sen Digo Gs * Eleourite Comping

Sin Onofro Nuclear Generalln Sintion tink 2.4 Unit 3,4

Souvtern Califomin Bditan Co.

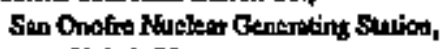
Unh 1.28

Sodivm Reacior Experiunerk, SRE, 31

Southera Nookar Operalling Co., Joseph WC.

Forky Nualear Finl

Uull I, 3

Uinil 23

Soallwal Atomia Bnery Assoctwol, Sowhured Buperimentid Pasl Oxido Ronolor, SBFOR, 3

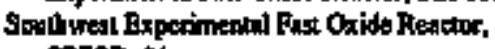
SEFOR, 31

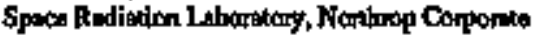

Lebontorito, TRIOA +4k E, 37

SPADHFTSE, SSTWSS, II

Spain

Alneme

Unit 1, XR-1088, 17 Atoo

Unik J, XR-090, 17

Unik 2, XR-OBg, 17

Cofrentos, Utail 1, XR-097, 17

Io요 Cobrern, XR-0.99, 17 


\section{Spatin (Continued)}

Lemonis

Unit I, XR-039, 18

Unit $2, X_{R}-0899,18$

Wucker Fineny Bourd J50, XR-010, 20

Swar Mutis of Gecons, XR-064, I7

Valderabellonot

Unit 1, XR-110, 18

Uat: 2, XR-110, 1

Vandello, Un' 2, XR-122, I?

Speció Powt Erearsion Reaclor Tat

No. t. 35

No, 2, 35

No. 4,35

SPRRT-1 (Specin! Power Fireorion Ractor), 3s SPERT-2 (Specil Power Rixcortion Resctor), 35 SFERT-3 (Specid Power Frevetion Reactor), 35 SPERT-4 (Apecinl Powar Ercarion Rewotor), 35

SFR (Nondin Polsed Reation), 38

STR-II (Stad dia Pulped Reactor), 8

SPR-III (Sindio Pilud Reaciorit,

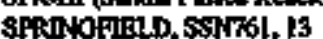

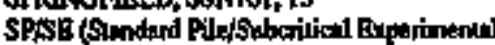
Complan), 42

SR 305-jA Ten Pilt, Tent File, 42

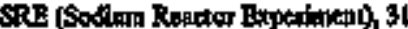

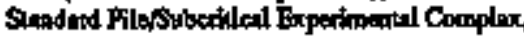
SPSBE 42

Sinford Univertily, 39

Stal Univertily of Now York, PULSTAR, 9 Sinlianacy Low Power Pint No. 1, SL-1, dS

Stulienary Medium Fower Pim

16. 1, SM-1, 43

$$
\text { No. } 1 \text { A SM-LA, } 43
$$

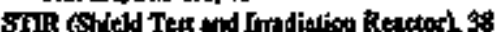

STONEWALL IACKSON, SSHN634, 4\%

Sudwik AB, Swoden, XR +019, 19

STUROFCN, Sstusti, 44

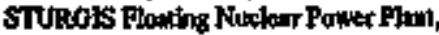
MP $-I A, 43$

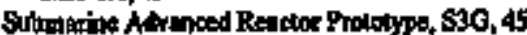
Sybratoine Inleonedinte Reactor Mint $A$ 510,45

Subranime Thernal Rexctor Pacilly, S1W, 45 Stenner Nutlar Simtion, Unh I, 5

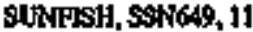

Super KCJKI $A_{1} 46$

supo (soper Porver Waror Boikd 37

Sung Pow Siation

Unit 1,5

Unil 2,
Surquehinin Stem Flectric Szation

1,5

Unt 2,5

Swetos, Pingtals

Unit 2, XR - 069,17

Gnit 3, XR-10\%5, 17

Init 4, XR-103, 17

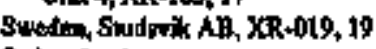

Swlicotand

Batad, Uhivanity of, 22

Berrin

Unt 1, XR $-063,17$

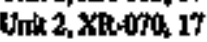

Gavew, Unitysily of, 47

Lofosind, XR-104, I7

Asulitebest, XR-061, 17

Proll Schemer Intibuta, 20

SWORDFISH, SSNSTS, 43

Twitran Power Co.

Chinghen

Unk 1, XR-080, 17

Uni 2 XR Xos, 17

Kuo Sineng

Unit 1, XR-096, 17

Unit 2, XR-09s, 17

Magnohan

Ink L, XR-113, 17

Uhik 2, XR-113, 17

Toimen

Uhitt, 18

than 8 , 18

TAUTOC, SSNG9, I

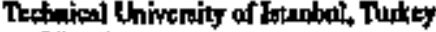

$\mathrm{XR}-[\mathrm{LBB}, 2]$

Techrtical Univatiny of Bunich, Getmany, ERM Geanchine, XR+004, 21

TECUMSER, SSENt628,44

Tehme, Uhiveriny of Jin, XR-0R, 2

TEANNESSEB SSBN734, 12

Toundeves Valloy Aulhort'ly

Bellefoots Sucter Plun Unit 1,5 Unit 2.5

Brown Peroy Nuclear Rower Sintion Unil 1, 1

Uni 2,1

Unit 3,1

Seqpoyah Nudear Plant

Unis 1,4

Uhat 2,4
Ternesse Vuitoy Auhotky (Conoinues) Wall Bar Nocdaser Pbal Unit 1,5 thil 25

This and Embution Commind, USA Anny Palo Radiation Fud'lity, APRF, is

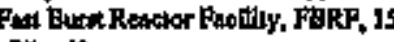

Tow Piln $n_{1} 42$

JEXAS (2 reation), CONH9. 45

Toxas AAM Uativertily AGIN-201M-105,

$$
\text { TRKA } 9
$$

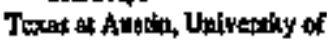
TFIOA-ME I, 40

$$
\text { TRGAAM II, } 9
$$

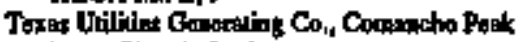
Siengen Btectrits Strilion

Whit 1,2

the 3

Theilond, TRR-1, XR-112, 21

THEODORE ROOSEVELT (2) Toolon? (anT1 13

THEODORB ROOSEVELT, SSBWEOO, 43

Themanl Tet Rescior Na. 1, TIR-1, 46

THOMAS A. BDISON, SSN610, 4

THOMAS JEFPERSON, SSN618, 44

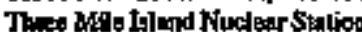

Unit 1,5

Uath 2, 28

THRESPER, SSN598, 4

TINOSA, SSN606, 4

Toko Eledric Power Co

Foludina Dodithi Power Sistion Uhil 14 XR-066, 16 Unth 2 XR-012, 16 Uhit 6 , XR-084, 16

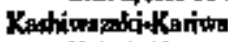
Whit 6 , 10

TOLEDO, SSN769, 13

Taledo Bdicon Co. Dawte-Bento Nuclatr Powar Simtion, Unit: 1,2

TOPEKA, SSNFS4

Tomey Pines, TRUOA A Al II Reactor, 38

TORY IIA, 45

TORY IIC, 45

Towtrshielding Reaclor

T\&R, 3B

TSR-2, 34
Trandiont Reantion Tent Fualliy, TREAT, 38 TREPANC, SSTW74, II

Triden Proviypa, sec, 14

Ttiok

Marytend, Untweraly of,

Teral AdM UnWerility, 9

Wigconain, Uhiwanity of, 10

TRFuA-Fi.P, Puento Rico Nivilear Cener, 40 TRIGA

Genord Atomict, Advinsed TRIGA-Ma P Procaypo Rodector, T

Honterop Corporaic Leborstotios, 97 TRJGA-MkI

Arizans, Uhiversidy of,

Cobiomin, Livtina, Univerily of,

thom Coentcel Ca, Dow Cheninal Ca, ?

Geredil Atonica, TRIOA tik 1 Protokypo Resctor, ?

Miehigan Siste Univeretios, 40

Ontha Yeannom Adminituration Hodpiilal T

Reed Collegro, 9

Texal an Andion, Untwerity of, 40

U.\$. Gexlojical Sarvay Laborntory. 8

Unh, Univatity of, 10

TRFOA Mk II

Columbla Universily, 39

Camnif University,

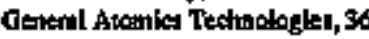

Illibedil, Utivetitity of, :

Kunsar Sine Un'verticy, 9

Texas at Anciln, Univerrity of,

TRIGA Mak III

Cvliomin, Benkeky, Unbersity of, 39

Toersy Pints, IRISA +Mk FI Resolor, 3B

TRTION (2 Rewstors), SSN 585,43

Trojen Nuctear PJ En1, Undil 1, 28

TRUXTUN (2 roackert), CON35, A5

TSR. Tower Shiclding Rencort, 38

TSR-2, Touver Shielding Reockor No. II, 38

TJR-1, Themal Tost Reactor No, 1, 16

TJR-2, Thomal Tou Reactor No, 2, 38

TUCSON, SSNTHO, 13

TULLLAEE, SSNL9?, 43

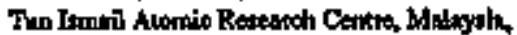
XR-125, 20

TUNONY, SSNTt62, 11

Turkty

Atomis Brargy Comtmission, XR-O9D, 2

Inunbod, Techotical Uhivertiky of, XR-164, 21 
Tudkey Poind Pint

Unt 3,5

Unit 4,5

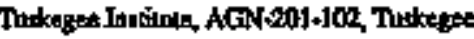
Btotiture, 40

UFIR Univerimy of Fodin Tar1 Reactor), 8 UHTREX, 31

ULR (Uhtrensily of Lowall Reactor), 9

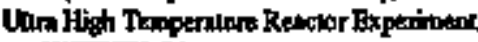
UHIREX, 31

ULYSSBS S, GRANT, SSBN631, 4

UMR-R (Untworalty of Mlbeond oIRoild. Rercior), 9

Union Etectriat, S.A. Spain Almares Utht L, XR-0he, 17 Uait $2, X_{\text {RR-068, } 17}$

Jose Cubrem, XP-05\%, IT

Union Bloctic Co, Collaway Minti, Unil 1,1

Unived Kingtow, Queen Mary Collega, 10 andoen Untuvernity, XR-04\%, AT

Uniled Ninclear Corpontion

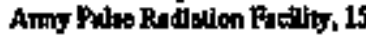

Devalicponem Divition, PTR,

Proling Reatearch Rescoor, 38

Uhiversitios of Finkfun and Damatidi, Getpnomy, XR-009, 47

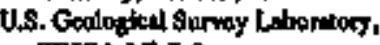
TRFOA-H: I, 8

Utuak Unlventis of

ACN1201-[07, 4]

TrRTA-Mat It it

UTR-1,35

UTR-10 (Iown San Uithersily) 8

UTR-10 (Vinida Polytochnda faxitoloh, 41

UTR Tex Reanis, 38

UVAR (Uinlwestity of Vireinin), 10

Vallecilos Holling Wuter Resclor, YBWR, 31

Valentid AB, Swodon, Ringhaly

Ua'k 2, X32-069, 17

Unis 3, XR-095, 17

Unin 4, XR-I0s, IT

YBWR (Yitedisi Boiling Wuer Reactor), 31

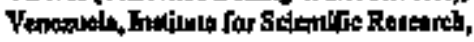
XR-0I8, 2]

Vemin, 48

Vemoon Yankee Ntuder Power Comp Venont

Yalkeo Muctear Powar sinitim, 5

Venketispith Thabe, 48

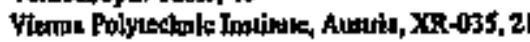

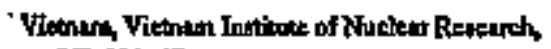
$X R, 032,47$

Yigg C. Sommer Nackear Sustion, Unh 1.5

VIRGLWLA (2 tesctors), CONNSE, 45

Vigititi, Univenity of

CAYALIER, 41

UVAR, 10

Virginta Brearle \& Pows Co.

roonh Antin Petwer Sustion

Unil 1,3

Inis 3

Souny Poner Station

Unit I,5

Unit 2,5

Vinginin PotyrectonlcImtilute, 1TTR-jo, 4

Viso Corpontion of America, Comall Univonits Zeto Powrer Reachor, 8

Voullo Nuclen Fower Plat

Unia 1,1

Unit 2, ]

VON STEUAFiN, SSANG02, 44

Waler Reed Anny Institole of Ressard, Wulk Beod Rupearch Howctor, WRRR, 46

Willet Hoed Retetath Retclot, WRRR, 4

Wathington, Unlwemty of, Etincalor, 41

Wastalagion Revelear Project

Uank 1,5

Unte 2,5

Wathiagon Pablic Power Supply Syaber N Rewior, 28

Wuhington Strot Univenity, WSTR, 10

Witer Reflector Tank, 48

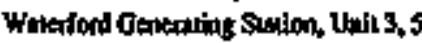

Wout Bar Ruckar Plant

Unith 1,5

Utrit 2,5

WEST VRRORU $A$, SSBN736, 12

Wou VIrgin Unlven'ky, ACN-211-109, 4

Waulinghouro Elotrio Comportion

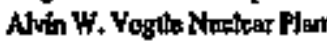
Unit 1, 1

Unbi 2, 1

Boaver Volloy Power Siction

unit 1

Ihix 2.1

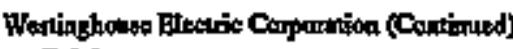
Hekiginn

Doel

Unih 1, 15

Unit 2,15

Unil 4, 15

Thmen, thit 3,15 .

Anidwood Station

Unt 1, 1

Uhit 2, 1

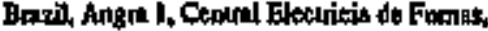
15

Byton Sintion

Uotk 1, I

Unt 2, :

Gijswhy Plent, Unis 1,

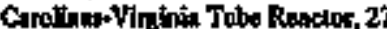

Carmubs Nucterer Station

Unik 1, 1

Utik 2,2

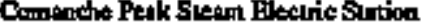

Init 1.2

Unh 2, 2

Diable Conyon Nucker Power Panl

tinit 1,2

Doridd C. Cook Nutisar Powar Plant

Unit 1,2

Unit 2.2

DRE ADNOUGHT, 4

Eagind, stzemell B, Is

Girew Bituid, SSW for HMS

Heddind Nock Pinnt, 2

H.B. Rebinoson Plumt, Unall 2, 2

Intitm Point Straion

Uni, 2, 2

Unhis 3,3

Iiniy, Thino Vetecllen, 46

Juon

Minuma Power Siation, Unit 1, 16

Oti Power Sintion

tnit 1, to

In't 2 , 16

Jotegh H. Fatley Nudeur Pint

this 1,3

Jnit2, 3

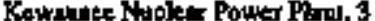

Westinghame Electrit Corponditun (Continuel) Kose:

Koil1, t6

Kori2, th

Konts, 16

Kort-4, 16

Youngswang-1, 16

Yongguang 2,16

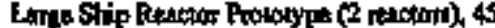

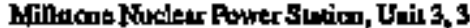

N Reacter, 28

Nawnal Ciratanion Tost Plank, is

Nonth Anat Power Sietion

Unit i, 3

Uhis 3

Fuctear Roctet Engint Rescier Experiment (NERVA), 32

Phillopinen, Repubtito of the Pailipine Nackent Powes Platil, Uhil I, 18

Poibl Beach Nackear Flan:

Unill 1,4

$$
\text { Un'i } 2,4
$$

Prilite Jalund Nivolew Generwing Plant Unt 1,4 tintil 24

Robert Emment Citon Nuctent Power Pint. Unit 1,4

Salem Nuctar Geanrting Strtion Unix 1, 4 Unit 2, 4

Sin Onofre NuclearGuoretiag Sution Und 1,28

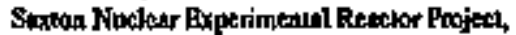
31

Serbrook Nucterc Siwlon

Unit 1, 4

Unit 1,4

Unit 2,4

Shempon Herit Nhriegr Power Plant Unit 1,4

Shippingport Atomitc Power Station, 20

Stovanta, Kniko, 17

Sowh Texis Projert

Unik l, 4

spaln.

A'mara

Unit 1, 17

Unis 2, 17 


\section{REACTOR INDEX (Continued)}

Weatingause Hleadic Corpotwlon (Conl'med) Spain (Continned)

A 100

Unith 1,17

Unin 2,17

Jow Cibren, 17

Lemoniz

$$
\text { Un't 1, } 10
$$

Vendellos, Unil 2, 17

Stbmitibe Themnal Reaclos Faolitly, 45

Swry Powrer Sintiton

$$
\text { Unth 1,5 }
$$

$$
\text { Unit 2,5 }
$$

sweden, Ringtad

Unto 2, 17

Untt 3, 17

1hit 4,17

Swizerinad, Beanul

$$
\text { 1his } 1,17
$$

Unik 2, IT

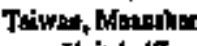

Li: 1,17
Wentighowre Blectrio Corporwion (Con hwed)

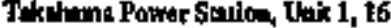

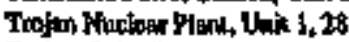

The -ire Poine Prem

Unit 3,5

$$
\text { Unit 4, } 5
$$

Vugla C. Somroer Nuokar Stution, Unit L, S

Wall Bar Haclese Plun:

$$
\text { Unil } 1,5
$$

Weatibghonse Nucleur Tesining Ceniar, 38

Whilioghouse Fucledr Tesining Cenlar,

$$
\text { Unit 1,5 }
$$$$
\text { thit } 2,5
$$

Woy Cuek Gertenting Shation, 5

Yankoo Nuoketr Power Stalion, 28

Zlon Nuclest Plant

$$
\text { Upít 1, }
$$

Weating bouke Hanford Co.

Fax Fiur Teat Prodity, 33

Nculcor Redilogeyphy Faclliny, 97

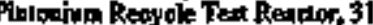

Westinghooso kivelesr Trainien Conler, WNTR,
Wotinghoure Tesling Recusio, WTR, 39

WHATB, SSN1630, 4

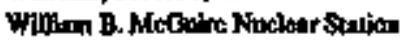

tinit 1,5

Unit 2,5

WLUUAM Et, BATES, SSN680, 11

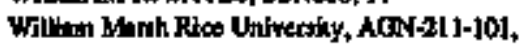

$$
41
$$

WHL.ROGERS, S\$BN659,44

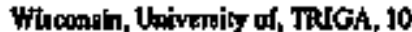

Wheowin Elestric Ponur Co.

Poink Bonth Nitclear Planl

$$
\text { toil 1, } 4
$$

$$
\text { tuin 2,4 }
$$

Whaconuin Pablic Soretes Corp. Kersaunee

Nuckear Rowor Plant, 3

WWTR (Woglingtrowse Nuclent Tyointing Beteina), 38

Wol Creak Gentating Sulions 5

WODDROW WILSON, SSBN624,

Wopposice Podyectiolo Inimilinte, 10

World Apricaliural Eair - U.S. Fixblbit Reactor, TRKLA-Ak II, 36
WRPR (Wiluer Reed Resernch Reatlot, 45

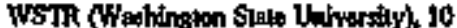

WTR(Weslinghoute Test Renctor), 3 4

WYOMING, SSEN7212, 13

Wroming, Untwordity of, $1, m, 41$

XE-Bıckup, 32

XE-Prínt, 32

Yauket Alomio Flectric Co, Yarkes Nucletr Power Stuition, 28

Yalkeo Nucker Powor Station, 28

Zgtre, Reglonel Cester for Nonkeur Surdici, 2

Zewo Poprex Physios Reactor, ZPPR, df

Zero Power Rewior

N6. 6, ZPR-6, 48

Na, 9,2PR-9,48

Zon Nuokar Pinl

14it 1,5

Jah 2,5

ZPPR (Zero Power Phyrios Recotor), 48

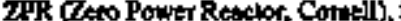

